\title{
PROJECTIVITY AND BIRATIONAL GEOMETRY OF BRIDGELAND MODULI SPACES
}

\author{
AREND BAYER AND EMANUELE MACRÌ
}

\begin{abstract}
We construct a family of nef divisor classes on every moduli space of stable complexes in the sense of Bridgeland. This divisor class varies naturally with the Bridgeland stability condition. For a generic stability condition on a K3 surface, we prove that this class is ample, thereby generalizing a result of Minamide, Yanagida, and Yoshioka. Our result also gives a systematic explanation of the relation between wall-crossing for Bridgeland-stability and the minimal model program for the moduli space.

We give three applications of our method for classical moduli spaces of sheaves on a K3 surface:

1. We obtain a region in the ample cone in the moduli space of Gieseker-stable sheaves only depending on the lattice of the K3.

2. We determine the nef cone of the Hilbert scheme of $n$ points on a K3 surface of Picard rank one when $n$ is large compared to the genus.

3. We verify the "Hassett-Tschinkel/Huybrechts/Sawon" conjecture on the existence of a birational Lagrangian fibration for the Hilbert scheme in a new family of cases.
\end{abstract}

\section{Contents}

1. Introduction 2

2. Review: Bridgeland stability conditions 8

3. Positivity 11

4. A natural nef divisor class on the moduli space, and comparison 15

5. Review: Moduli spaces for stable sheaves on K3 surfaces 17

6. Review: Stability conditions and moduli spaces for objects on K3 surfaces 20

7. K3 surfaces: Projectivity of moduli spaces 24

8. Flops via wall-crossing 28

9. Stable sheaves on K3 surfaces 30

10. Hilbert scheme of points on K3 surfaces 38

$\begin{array}{ll}\text { References } & 46\end{array}$

2010 Mathematics Subject Classification. 14D20, (Primary); 18E30, 14J28, 14E30 (Secondary).

Key words and phrases. Bridgeland stability conditions, Derived category, Moduli spaces of complexes, Mumford-Thaddeus principle. 


\section{INTRODUCTION}

In this paper, we give a canonical construction of determinant line bundles on any moduli space $M$ of Bridgeland-semistable objects. Our construction has two advantages over the classical construction for semistable sheaves: our divisor class varies naturally with the stability condition, and we can show that our divisor is automatically nef.

This also explains a picture envisioned by Bridgeland, and observed in examples by Arcara-Bertram and others, that relates wall-crossing under a change of stability condition to the birational geometry and the minimal model program of $M$. As a result, we can deduce properties of the birational geometry of $M$ from wall-crossing; this leads to new results even when $M$ coincides with a classical moduli space of Gieseker-stable sheaves.

Moduli spaces of complexes. Moduli spaces of complexes first appeared in [Bri02]: the flop of a smooth threefold $X$ can be constructed as a moduli space parameterizing perverse ideal sheaves in the derived category of $X$. Recently, they have turned out to be extremely useful in Donaldson-Thomas theory; see [Tod11] for a survey.

Ideally, the necessary notion of stability of complexes can be given in terms of Bridgeland's notion of a stability condition on the derived category, introduced in [Bri07]. Unlike other constructions (as in [Tod09, Bay09]), the space of Bridgeland stability conditions admits a well-behaved wall and chamber structure: the moduli space of stable objects with given invariants remains unchanged unless the stability condition crosses a wall. However, unlike Gieseker-stability for sheaves, Bridgeland stability is not a priori connected to a GIT problem. As a consequence, while established methods ([Ina02, Lie06, Tod08, AP06]) can prove existence of moduli spaces as algebraic spaces or Artin stacks, there are so far only ad-hoc methods to prove that they are projective, or to construct coarse moduli spaces.

In this paper, we propose a solution to this problem by constructing a family of numerically positive divisor classes on any moduli space of Bridgeland-stable complexes.

A family of nef divisors on Bridgeland-moduli spaces. Let $X$ be a smooth projective variety over $\mathbb{C}$. We denote by $\mathrm{D}^{\mathrm{b}}(X)$ its bounded derived category of coherent sheaves, and by $\operatorname{Stab}(X)$ the space of Bridgeland stability conditions on $\mathrm{D}^{\mathrm{b}}(X)$, see Section 2 . We refer to p. 8 for an overview of notations.

Let $\sigma=(Z, \mathcal{A}) \in \operatorname{Stab}(X)$ be a stability condition, and $v$ a choice of numerical invariants. Assume that we are given a family $\mathcal{E} \in \mathrm{D}^{\mathrm{b}}(S \times X)$ of $\sigma$-semistable objects of class $v$ parameterized by a proper algebraic space $S$ of finite type over $\mathbb{C}$; for example, $S$ could be a fine moduli space $M_{\sigma}(v)$ of stable objects. We define a numerical Cartier divisor class $\ell_{\sigma, \mathcal{E}} \in N^{1}(S)=\operatorname{Hom}\left(N_{1}(S), \mathbb{R}\right)$ as follows: for any projective integral curve $C \subset S$, we set

$$
\ell_{\sigma, \mathcal{E}}([C])=\ell_{\sigma, \mathcal{E}} \cdot C:=\Im\left(-\frac{Z\left(\Phi_{\mathcal{E}}\left(\mathcal{O}_{C}\right)\right)}{Z(v)}\right)=\Im\left(-\frac{Z\left(\left.\left(p_{X}\right)_{*} \mathcal{E}\right|_{C \times X}\right)}{Z(v)}\right)
$$

where $\Phi_{\mathcal{E}}: \mathrm{D}^{\mathrm{b}}(S) \rightarrow \mathrm{D}^{\mathrm{b}}(X)$ is the Fourier-Mukai functor with kernel $\mathcal{E}$, and $\mathcal{O}_{C}$ is the structure sheaf of $C$. It is easy to prove that (1) defines a numerical divisor class $\ell_{\sigma, \mathcal{E}} \in$ $N^{1}(S)$. Our main result, the Positivity Lemma 3.3, implies the positivity of this divisor: 
Theorem 1.1. The divisor class $\ell_{\sigma, \mathcal{E}}$ is nef. Additionally, we have $\ell_{\sigma, \mathcal{E}} \cdot C=0$ if and only if for two general points $c, c^{\prime} \in C$, the corresponding objects $\mathcal{E}_{c}, \mathcal{E}_{c^{\prime}}$ are $S$-equivalent.

(Two semistable objects are $S$-equivalent if their Jordan-Hölder filtrations into stable factors of the same phase have identical stable factors.) The class $\ell_{\sigma, \mathcal{E}}$ can also be given as a determinant line bundle. The main advantage of our construction is that we can show its positivity property directly, without using GIT; instead, the proof is based on a categorical construction by Abramovich and Polishchuk [AP06, Pol07]. Our construction also avoids any additional choices: it depends only on $\sigma$.

Chambers in $\operatorname{Stab}(X)$ and the nef cone of the moduli spaces. Consider a chamber $\mathcal{C}$ for the wall-and-chamber decomposition with respect to $v$; then (assuming its existence) the moduli space $M_{\mathcal{C}}(v):=M_{\sigma}(v)$ of $\sigma$-stable objects of class $v$ is constant for $\sigma \in \mathcal{C}$. Also assume for simplicity that it admits a universal family $\mathcal{E}$. Theorem 1.1 yields an essentially linear map

$$
l: \overline{\mathcal{C}} \rightarrow \operatorname{Nef}\left(M_{\mathcal{C}}(v)\right), \quad l(\sigma)=\ell_{\sigma, \mathcal{E}}
$$

This immediately begs for the following two questions:

Question 1: Do we actually have $l(\mathcal{C}) \subset \operatorname{Amp}\left(M_{\mathcal{C}}(v)\right)$ ?

Question 2: What will happen at the walls of $\mathcal{C}$ ?

K3 surfaces: Overview. While our above approach is very general, we now restrict to the case where $X$ is a smooth projective K3 surface. In this situation, Bridgeland described (a connected component of) the space of stability conditions in [Bri08], and Toda proved existence results for moduli spaces in [Tod08]; see Section 6. The following paraphrases a conjecture proposed by Bridgeland in Section 16 of the arXiv-version of [Bri08]:

Conjecture 1.2 (Bridgeland). Given a stability condition $\sigma$ on a K3 surface, and a numerical class $v$, there exists a coarse moduli space $M_{\sigma}(v)$ of $\sigma$-semistable complexes with class $v$. Changing the stability condition produces birational maps between the coarse moduli spaces.

Our main results give a partial proof of this conjecture, and answers to the above questions: Theorem 1.3 answers Question 1 and proves existence of coarse moduli spaces; Theorem 1.4 partially answers Question 2 and partially proves the second statement of the conjecture. They also give a close relation between walls in $\operatorname{Stab}(X)$, and walls in the movable cone of the moduli space separating nef cones of different birational models.

Projectivity of the moduli spaces. Assume that $\sigma$ is generic, which means that it does not lie on a wall with respect to $v$.

Theorem 1.3. Let $X$ be a smooth projective $K 3$ surface, and let $v \in H_{\mathrm{alg}}^{*}(X, \mathbb{Z})$. Assume that the stability condition $\sigma$ is generic with respect to $v$. Then:

(a) The coarse moduli space $M_{\sigma}(v)$ of $\sigma$-semistable objects with Mukai vector $v$ exists as a normal projective irreducible variety with $\mathbb{Q}$-factorial singularities.

(b) The assignment (1) induces an ample divisor class $\ell_{\sigma}$ on $M_{\sigma}(v)$. 
This generalizes [MYY11b, Theorem 0.0.2], which shows projectivity of $M_{\sigma}(v)$ in the case where $X$ has Picard rank one.

Wall-crossing and birational geometry of the moduli spaces. We also use Theorem 1.1 to study the wall-crossing behavior of the moduli space under deformations of $\sigma$.

Assume that $v$ is a primitive class. Let $W$ be a wall of the chamber decomposition for $v$. Let $\sigma_{0}$ be a generic point of $W$, and let $\sigma_{+}, \sigma_{-}$be two stability conditions nearby on each side of the wall. By Theorem 1.3 and its proof, they are smooth projective Hyperkähler varieties. Since being semistable is a closed condition in $\operatorname{Stab}(X)$, the (quasi-)universal families $\mathcal{E}_{ \pm}$on $M_{\sigma_{ \pm}}(v)$ are also families of $\sigma_{0}$-semistable objects. Theorem 1.1 also applies in this situation, and thus $\sigma_{0}$ produces nef divisor classes $\ell_{\sigma_{0}, \mathcal{E}_{ \pm}}$on $M_{\sigma_{ \pm}}(v)$. In Section 8 , we prove:

Theorem 1.4. Let $X$ be a smooth projective $K 3$ surface, and $v \in H_{\mathrm{alg}}^{*}(X, \mathbb{Z})$ be a primitive class.

(a) The classes $\ell_{\sigma_{0}, \mathcal{E}_{ \pm}}$are big and nef, and induce birational contraction morphisms

$$
\pi_{\sigma_{ \pm}}: M_{\sigma_{ \pm}}(v) \rightarrow Y_{ \pm}
$$

where $Y_{ \pm}$are normal irreducible projective varieties.

(b) If there exist $\sigma_{0}$-stable objects, and if their complement in $M_{\sigma_{ \pm}}(v)$ has codimension at least two 2, then one of the following two possibilities holds:

- Both $\ell_{\sigma_{0}, \mathcal{E}_{+}}$and $\ell_{\sigma_{0}, \mathcal{E}_{-}}$are ample, and the birational map

$$
f_{\sigma_{0}}: M_{\sigma_{+}}(v) \rightarrow M_{\sigma_{-}}(v) \text {, }
$$

obtained by crossing the wall in $\sigma_{0}$, extends to an isomorphism.

- Neither $\ell_{\sigma_{0}, \mathcal{E}_{+}}$nor $\ell_{\sigma_{0}, \mathcal{E}_{-}}$is ample, and $f_{\sigma_{0}}: M_{\sigma_{+}}(v) \rightarrow M_{\sigma_{-}}(v)$ is the flop induced by $\ell_{\sigma_{0}, \mathcal{E}_{+}}$: we have a commutative diagram of birational maps

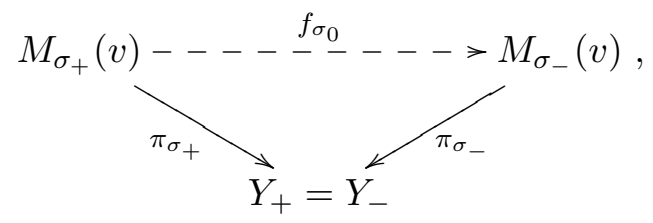

and $f_{\sigma_{0}}^{*} \ell_{\sigma_{0}, \mathcal{E}_{-}}=\ell_{\sigma_{0}, \mathcal{E}_{+}}$.

Note that our theorem does not cover the cases where there are no $\sigma_{0}$-stable objects, or the case where their complement has codimension one ${ }^{1}$

In some examples (including those considered in [AB13, MM13]), we can show that $Y_{+}=Y_{-}$is a connected component of the coarse moduli space of $\sigma_{0}$-semistable objects of class $v$. The contraction from the moduli space of Gieseker-stable sheaves to the Uhlenbeck moduli space of $\mu$-semistable sheaves (see [LP92, Li93]) is another particular example of the contraction morphism $\pi_{\sigma_{ \pm}}$; this was observed on the level of sets of semistable objects in [LQ11], and on the level of moduli spaces in the recent preprint [Lo12].

We study many more examples of wall-crossing behavior in Sections 9 and 10 .

\footnotetext{
${ }^{1}$ Our only result in the latter case is Lemma 10.10 , which shows $f_{\sigma_{0}}^{*} \ell_{\sigma_{0}, \mathcal{E}_{-}}=\ell_{\sigma_{0}, \mathcal{E}_{+}}$.
} 
Nef cones of moduli spaces of stable sheaves. Our Theorem 1.1 can give new results on the nef cone of $M_{\sigma}(v)$ even in the situation where it agrees with a classical moduli space of sheaves. We give two examples of such applications:

- In Corollary 9.14, we determine a region of the ample cone of the moduli space of Gieseker-stable sheaves on $X$ that depends only on the lattice of $X$.

- In Proposition 10.3, we determine the nef cone of the Hilbert scheme of $n$ points on a K3 surface of Picard rank one and genus $g$ for $n \geq \frac{g}{2}+1$. Our result shows in particular that [HT10, Conjecture 1.2] will need to be modified (see Remark 10.4).

The strength of our approach is that it simultaneously produces nef divisors (by Theorem 1.1) and extremal rays of the Mori cone (as curves of $S$-equivalent objects on the wall).

Lagrangian fibrations for the Hilbert scheme. Let $X$ be a K3 surface with Pic $X=$ $\mathbb{Z} \cdot H$, and of degree $d=\frac{1}{2} H^{2}$. We consider the Hilbert scheme $\operatorname{Hilb}^{n}(X)$ of $n$ points on $X$. According to a long-standing conjecture for Hyperkähler varieties, it has a birational model admitting a Lagrangian fibration if and only if $d=\frac{k^{2}}{h^{2}}(n-1)$ for some integers $k, h$. The following theorem solves the conjecture in the case $h=2$, and interprets all birational models as moduli spaces of Bridgeland-stable objects.

We denote by $\tilde{H} \subset \operatorname{Hilb}^{n}(X)$ the divisor of subschemes intersecting a given curve in the linear system $|H|$, and by $B \subset \operatorname{Hilb}^{n}(X)$ the reduced divisor of non-reduced subschemes.

Theorem 10.8. Let $X$ be a K3 surface with $\operatorname{Pic} X=\mathbb{Z} \cdot H$ and $H^{2}=2 d$. Assume that there is an odd integer $k$ with $d=\frac{k^{2}}{4}(n-1)$ for some integer $n$. Then:

(a) The movable cone $\operatorname{Mov}\left(\operatorname{Hilb}^{n}(X)\right)$ is generated by $\tilde{H}$ and $2 \tilde{H}-k B$.

(b) The morphism induced by $\tilde{H}$ is the Hilbert-to-Chow morphism, while the one induced by $2 \tilde{H}-k B$ is a Lagrangian fibration on a minimal model for $\operatorname{Hilb}^{n}(X)$.

(c) All minimal models for $\operatorname{Hilb}^{n}(X)$ arise as moduli spaces of stable objects in $\mathrm{D}^{\mathrm{b}}(X)$ and their birational transformations are induced by crossing a wall in $\operatorname{Stab}^{\dagger}(X)$.

\section{Some relations to existing work.}

Wall-crossing. Our construction was directly inspired by the results of [ABCH13]. The authors studied wall-crossing for the Hilbert scheme of points on $\mathbb{P}^{2}$, and found a surprisingly direct relation between walls $\operatorname{Stab}\left(\mathbb{P}^{2}\right)$ and walls in the movable cone of the Hilbert scheme separating nef cones of different birational models.

In their case, the variation of moduli spaces can also be seen as a variation of GIT parameters, via the classical monad construction. More precisely, stable complexes with respect to a Bridgeland stability condition can be seen as $\theta$-stable representations of a Beilinson quiver for $\mathbb{P}^{2}$, in the sense of King [Kin94]. In this case, our divisor class $\ell_{\sigma}$ agrees with the ample divisor coming from the affine GIT construction of these moduli spaces. More generally, our family of nef divisors generalizes a construction by Craw and Ishii in [CI04] that produces a family of nef divisors on moduli spaces of $\theta$-stable quiver representations. 
The analogue of Theorem 1.3 for abelian surfaces has been proved in [MYY11b]; a different method to prove projectivity was established in [MM13, Mac12].

Our main result implies that knowing the precise location of walls in $\operatorname{Stab}(X)$ has immediate applications to the geometry of the nef cone and the movable cone of the moduli spaces of stable objects. Various authors have considered the geometry of walls in $\operatorname{Stab}(X)$ explicitly: first examples were found by Arcara and Bertram in [AB13, AB11]; the case where $X$ is an abelian surface has been studied by Minamide, Yanagida and Yoshioka in [MYY11a, MYY11b, YY12], and by Meachan and Maciocia in [MM13, Mac12]; Lo and Qin studied the case of arbitrary surfaces in [LQ11]. As an example, our Corollary 9.14 is a straightforward combination of our main result and the main result of Kawatani in [Kaw11]; the above-mentioned authors had all used similar methods.

Proof of projectivity. In [MYY11a, MYY11b], the authors use a beautiful observation to reinterpret any moduli space of Bridgeland-semistable complexes on a K3 surface with Picard rank one as a moduli space of Gieseker-semistable sheaves on a Fourier-Mukai partner $Y$ of $X$. Our proof of Theorem 1.3 is directly based on a generalization of their idea to K3 surfaces of arbitrary Picard rank.

Strange duality. Our construction, and specifically Corollary 9.14 may prove useful for Le Potier's Strange Duality Conjecture, see [LP05] and [MO10]. While Le Potier's construction produces line bundles with sections, it is more difficult to show that these line bundles are nef; the Positivity Lemma can fill this gap.

Markman's monodromy operators. It would be very interesting to relate our picture to results by Markman on the movable cone in [Mar11]. Markman proves that the closure of the movable cone is a fundamental domain for a natural group action on the cone of big divisors. The group is generated by reflections, which presumably correspond to walls where $\ell_{\sigma_{0}, \mathcal{E}_{ \pm}}$induce divisorial contractions; we expect them to behave similarly to the "bouncing walls" appearing in Sections 9 and 10. The two maps $l_{ \pm}$of equation (2) for the two adjacent chambers can likely be identified via the monodromy operators introduced in [Mar08, Mar03].

Variation of GIT. The idea of varying a Bridgeland stability condition is a direct generalization of varying the polarization for Gieseker stability on sheaves. The latter was studied for surfaces in [EG95, FQ95, MW97], using variation of GIT [Tha96, DH98]. For K3 surfaces, the advantage of Bridgeland stability arises since the space of stability conditions has the correct dimension to span an open subset of the movable cone of every moduli space.

Lagrangian fibrations. As indicated above, Theorem 10.8 solves a special case of a conjecture on Lagrangian fibrations for arbitrary Hyperkähler varieties. This appeared in print in articles by Hassett-Tschinkel [HT01], Huybrechts [GHJ03] and Sawon [Saw03], and had been proposed earlier (see [Ver10]). In the case of the Hilbert scheme on a K3 surface with Picard rank one, it was proved for $d=k^{2}(n-1)$ independently by Markushevich [Mar06] and Sawon [Saw07], and the case of $d=\frac{1}{h^{2}}(n-1)$ by Kimura and Yoshioka [KY11]. Our proof is based on a Fourier-Mukai transform just as the proofs of Sawon and Markushevich; 
Bridgeland stability gives a more systematic tool to control generic preservation of stability under the transform.

Open questions. Theorem 1.4, and in particular case (b), does not treat the case of "totally semistable walls", which is the case where there is no $\sigma_{0}$-stable complex. Proving a similar result in general would lead to further progress towards determining the movable cone for moduli spaces of stable sheaves (for general results and conjectures on the movable cone for an Hyperkähler manifold, see [HT09, HT10]); in particular, it would likely imply the above-mentioned conjecture on Lagrangian fibrations for any moduli space of Giesekerstable sheaves on a K3 surface.

We will treat this case in [BM13].

Outline of the paper. Sections 2-4 treat the case of an arbitrary smooth projective variety $X$, while Sections 5-10 are devoted to the case of K3 surfaces.

Section 2 is a brief review of the notion of Bridgeland stability condition, and sections 3 and 4 are devoted to the proof of Theorem 1.1. The key ingredient is a construction by Abramovich and Polishchuk in [AP06, Pol07]: Given the t-structure on $X$ associated to a Bridgeland stability condition, their construction produces a t-structure on $\mathrm{D}^{\mathrm{b}}(S \times X)$, for any scheme $S$. This categorical ingredient allows us to transfer the basic positivity of the "central charge", see equation (3), to the positivity of $\ell_{\sigma, \mathcal{E}}$ as a divisor.

As indicated above, the second part of the paper is devoted to the case of K3 surfaces. Sections 5 and 6 recall background about Gieseker-stability and Bridgeland stability conditions, respectively.

We prove Theorem 1.3 in Section 7. We show projectivity of the moduli space by identifying it with a moduli space of semistable sheaves via a Fourier-Mukai transform, generalizing an idea in [MYY11b]. Section 8 contains the proof of Theorem 1.4. Since the moduli spaces are $K$-trivial and $\ell_{\sigma_{0}, \mathcal{E}_{ \pm}}$are nef by Theorem 1.1, it only remains to prove that they are big; the proof is based on Yoshioka's description of the Beauville-Bogomolov form on the moduli space in terms of the Mukai pairing of the K3 surface in [Yos01b].

The final two sections 9 and 10 are devoted to applications to moduli spaces of sheaves and to the Hilbert scheme, respectively.

Acknowledgements. The authors would like to thank in particular Aaron Bertram and Daniel Huybrechts for many insightful discussions related to this article; the first author would also like to thank Alastair Craw for very useful discussions on our main construction of nef divisors in different context. We are also grateful to Izzet Coskun, Alina Marian, Eyal Markman, Dragos Oprea, Paolo Stellari, and Jenia Tevelev for comments and discussions, to Eyal Markman and Kōta Yoshioka for very useful comments on an earlier version of this article, and to the referee for very helpful comments which greatly improved the exposition.

This project got started while the first author was visiting the programme on moduli spaces at the Isaac Newton Institute in Cambridge, England, and he would like to thank the institute for its warm hospitality and stimulating environment. The collaboration continued during a visit of both authors to the Hausdorff Center for Mathematics, Bonn, and we would like to thank the HCM for its support. A. B. is partially supported by 
NSF grant DMS-1101377. E. M. is partially supported by NSF grant DMS-1001482/DMS1160466, Hausdorff Center for Mathematics, Bonn, and by SFB/TR 45.

Notation and Convention. For an abelian group $G$ and a field $k(=\mathbb{Q}, \mathbb{R}, \mathbb{C})$, we denote by $G_{k}$ the $k$-vector space $G \otimes k$.

Throughout the paper, $X$ will be a smooth projective variety over the complex numbers. For a (locally-noetherian) scheme (or algebraic space) $S$, we will use the notation $\mathrm{D}^{\mathrm{b}}(S)$ for its bounded derived category of coherent sheaves, $\mathrm{D}_{q c}(S)$ for the unbounded derived category of quasi-coherent sheaves, and $\mathrm{D}_{S \text {-perf }}(S \times X)$ for the category of $S$-perfect complexes. (An $S$-perfect complex is a complex of $\mathcal{O}_{S \times X}$-modules which locally, over $S$, is quasi-isomorphic to a bounded complex of coherent shaves which are flat over $S$.)

We will abuse notation and denote all derived functors as if they were underived. We denote by $p_{S}$ and $p_{X}$ the two projections from $S \times X$ to $S$ and $X$, respectively. Given $\mathcal{E} \in \mathrm{D}_{q c}(S \times X)$, we denote the Fourier-Mukai functor associated to $\mathcal{E}$ by

$$
\Phi_{\mathcal{E}}\left(\_\right):=\left(p_{X}\right)_{*}\left(\mathcal{E} \otimes p_{S}^{*}\left(\_\right)\right) .
$$

We let $K_{\text {num }}(X)$ be the numerical Grothendieck group of $X$ and denote by $\chi(-)$ (resp., $\chi(-,-))$ the Euler characteristic on $K_{\text {num }}(X)$ : for $E, F \in \mathrm{D}^{\mathrm{b}}(X)$,

$$
\chi(E)=\sum_{p}(-1)^{p} h^{p}(X, E) \quad \text { and } \quad \chi(E, F)=\sum_{p}(-1)^{p} \operatorname{ext}^{p}(E, F) .
$$

We denote by $\mathrm{NS}(X)$ the Néron-Severi group of $X$, and write $N^{1}(X):=\mathrm{NS}(X)_{\mathbb{R}}$. The space of full numerical stability conditions on $\mathrm{D}^{\mathrm{b}}(X)$ will be denoted by $\operatorname{Stab}(X)$.

Given a complex $E \in \mathrm{D}^{\mathrm{b}}(X)$, we denote its cohomology sheaves by $\mathcal{H}^{*}(E)$. The skyscraper sheaf at a point $x \in X$ is denoted by $k(x)$. For a complex number $z \in \mathbb{C}$, we denote its real and imaginary part by $\Re z$ and $\Im z$, respectively.

For a K3 surface $X$, we denote the Mukai vector of an object $E \in \mathrm{D}^{\mathrm{b}}(X)$ by $v(E)$. We will often write it as $v(E)=(r, c, s)$, where $r$ is the rank of $E, c \in \mathrm{NS}(X)$, and $s$ the degree of $v(E)$. For a spherical object $S$ we denote the spherical twist at $S$ by $\mathrm{ST}_{S}\left(\_\right)$, defined in [ST01] by the exact triangle, for all $E \in \mathrm{D}^{\mathrm{b}}(X)$,

$$
\operatorname{Hom}^{\bullet}(S, E) \otimes S \rightarrow E \rightarrow \operatorname{ST}_{S}(E) \text {. }
$$

\section{Review: BRIDGElAnd STABILITy CONDitions}

In this section, we give a brief review of stability conditions on derived categories, as introduced in [Bri07].

Let $X$ be a smooth projective variety, and denote by $\mathrm{D}^{\mathrm{b}}(X)$ its bounded derived category of coherent sheaves. A full numerical stability condition $\sigma$ on $\mathrm{D}^{\mathrm{b}}(X)$ consists of a pair $(Z, \mathcal{A})$, where $Z: K_{\text {num }}(X) \rightarrow \mathbb{C}$ is a group homomorphism (called central charge) and $\mathcal{A} \subset \mathrm{D}^{\mathrm{b}}(X)$ is the heart of a bounded t-structure, satisfying the following three properties:

(a) For any $0 \neq E \in \mathcal{A}$ the central charge $Z(E)$ lies in the following semi-closed upper half-plane:

$$
Z(E) \in \mathbb{H}:=\mathcal{H} \cup \mathbb{R}_{<0}=\mathbb{R}_{>0} \cdot e^{(0,1] \cdot i \pi}
$$


This positivity condition is the essential ingredient for our positivity result. One could think of it as two separate positivity conditions: $\Im Z$ defines a rank function on the abelian category $\mathcal{A}$, i.e., a non-negative function rk: $\mathcal{A} \rightarrow \mathbb{R}_{\geq 0}$ that is additive on short exact sequences. Similarly, $-\Re Z$ defines a degree function $\operatorname{deg}: \mathcal{A} \rightarrow \mathbb{R}$, which has the property that $\operatorname{rk}(E)=0 \Rightarrow \operatorname{deg}(E)>0$. We can use them to define a notion of slope-stability with respect to $Z$ on the abelian category $\mathcal{A}$ via the slope $\mu(E)=\frac{\operatorname{deg}(E)}{\operatorname{rk}(E)}$.

(b) With this notion of slope-stability, every object in $E \in \mathcal{A}$ has a Harder-Narasimhan filtration $0=E_{0} \hookrightarrow E_{1} \hookrightarrow \ldots \hookrightarrow E_{n}=E$ such that the $E_{i} / E_{i-1}$ 's are $Z$ semistable, with $\mu\left(E_{1} / E_{0}\right)>\mu\left(E_{2} / E_{1}\right)>\cdots>\mu\left(E_{n} / E_{n-1}\right)$.

(c) There is a constant $C>0$ such that, for all $Z$-semistable object $E \in \mathcal{A}$, we have

$$
\|E\| \leq C|Z(E)|
$$

where $\|*\|$ is a fixed norm on $K_{\text {num }}(X) \otimes \mathbb{R}$.

The last condition was called the support property in [KS08], and is equivalent (see [BM11, Proposition B.4]) to Bridgeland's notion of a full stability condition.

Definition 2.1. A stability condition is called algebraic if its central charge takes values in $\mathbb{Q} \oplus \mathbb{Q} \sqrt{-1}$.

As $K_{\text {num }}(X)$ is finitely generated, for an algebraic stability condition the image of $Z$ is a discrete lattice in $\mathbb{C}$.

Given $(Z, \mathcal{A})$ as above, one can extend the notion of stability to $\mathrm{D}^{\mathrm{b}}(X)$ as follows: for $\phi \in$ $(0,1]$, we let $\mathcal{P}(\phi) \subset \mathcal{A}$ be the full subcategory $Z$-semistable objects with $Z(E) \in \mathbb{R}_{>0} e^{i \phi \pi}$; for general $\phi$, it is defined by the compatibility $\mathcal{P}(\phi+n)=\mathcal{P}(\phi)[n]$. Each subcategory $\mathcal{P}(\phi)$ is extension-closed and abelian. Its nonzero objects are called $\sigma$-semistable of phase $\phi$, and its simple objects are called $\sigma$-stable. Then each object $E \in \mathrm{D}^{\mathrm{b}}(X)$ has a HarderNarasimhan filtration, where the inclusions $E_{i-1} \subset E_{i}$ are replaced by exact triangles $E_{i-1} \rightarrow E_{i} \rightarrow A_{i}$, and where the $A_{i}$ 's are $\sigma$-semistable of decreasing phases $\phi_{i}$. The category $\mathcal{P}(\phi)$ necessarily has finite length. Hence every object in $\mathcal{P}(\phi)$ has a finite Jordan-Hölder filtration, whose filtration quotients are $\sigma$-stable objects of the phase $\phi$. Two objects $A, B \in \mathcal{P}(\phi)$ are called $S$-equivalent if their Jordan-Hölder factors are the same (up to reordering).

The set of stability conditions will be denoted by $\operatorname{Stab}(X)$. It has a natural metric topology (see [Bri07, Prop. 8.1] for the explicit form of the metric). Bridgeland's main theorem is the following:

Theorem 2.2 (Bridgeland). The map

$$
\mathcal{Z}: \operatorname{Stab}(X) \rightarrow \operatorname{Hom}\left(K_{\text {num }}(X), \mathbb{C}\right), \quad(Z, \mathcal{A}) \mapsto Z,
$$

is a local homeomorphism. In particular, $\operatorname{Stab}(X)$ is a complex manifold of finite dimension equal to the rank of $K_{\text {num }}(X)$.

In other words, a stability condition $(Z, \mathcal{A})$ can be deformed uniquely given a small deformation of its central charge $Z$. 
Let us now fix a class $v \in K_{\text {num }}(X)$, and consider the set of $\sigma$-semistable objects $E \in$ $\mathrm{D}^{\mathrm{b}}(X)$ of class $v$ as $\sigma$ varies. The proof of the following statement is essentially contained in [Bri08, Section 9]; see also [BM11, Proposition 3.3] and [Tod08, Prop 2.8]:

Proposition 2.3. There exists a locally finite set of walls (real codimension one submanifolds with boundary) in $\operatorname{Stab}(X)$, depending only on $v$, with the following properties:

(a) When $\sigma$ varies within a chamber, the sets of $\sigma$-semistable and $\sigma$-stable objects of class $v$ does not change.

(b) When $\sigma$ lies on a single wall $W \subset \operatorname{Stab}(X)$, then there is a $\sigma$-semistable object that is unstable in one of the adjacent chambers, and semistable in the other adjacent chamber.

(c) When we restrict to an intersection of finitely many walls $W_{1}, \ldots, W_{k}$, we obtain a wall-and-chamber decomposition on $W_{1} \cap \cdots \cap W_{k}$ with the same properties, where the walls are given by the intersections $W \cap W_{1} \cap \cdots \cap W_{k}$ for any of the walls $W \subset \operatorname{Stab}(X)$ with respect to $v$.

If $v$ is primitive, then $\sigma$ lies on a wall if and only if there exists a strictly $\sigma$-semistable object of class $v$. The Jordan-Hölder filtration of $\sigma$-semistable objects does not change when $\sigma$ varies within a chamber.

Definition 2.4. Let $v \in K_{\text {num }}(X)$. A stability condition is called generic with respect to $v$ if it does not lie on a wall in the sense of Proposition 2.3.

We will also need the following variant of [Tod08, Lemma 2.9]:

Lemma 2.5. Consider a stability condition $\sigma=(Z, \mathcal{A})$ with $Z(v)=-1$. Then there are algebraic stability conditions $\sigma_{i}=\left(Z_{i}, \mathcal{A}_{i}\right)$ for $i=1, \ldots, m$ nearby $\sigma$ with $Z_{i}(v)=-1$ such that:

(a) For every $i$ the following statement holds: an object of class $v$ is $\sigma_{i}$-stable (or $\sigma_{i}$-semistable) if and only if it is $\sigma$-stable (or $\sigma$-semistable, respectively).

(b) The central charge $Z$ is in the convex hull of $\left\{Z_{1}, \ldots, Z_{n}\right\}$.

Proof. If $v$ is generic, this follows immediately from Theorem 2.2 and Proposition 2.3, and the density of algebraic central charges $\operatorname{Hom}\left(K_{\text {num }}(X), \mathbb{Q} \oplus i \mathbb{Q}\right)$ inside the vector space $\operatorname{Hom}\left(K_{\text {num }}(X), \mathbb{C}\right)$. Once we restrict to the subset $Z(v)=-1$, any wall is locally defined by a linear rational equation of the form $\Im Z(w)=0$, where $w \in K_{\text {num }}(X)$ is the class of a destabilizing subobject, and thus the claim follows similarly.

Remark 2.6. There are two group actions on $\operatorname{Stab}(X)$, see [Bri07, Lemma 8.2]: the group of autoequivalences $\operatorname{Aut}\left(\mathrm{D}^{\mathrm{b}}(X)\right)$ acts on the left via $\Phi(Z, \mathcal{A})=\left(Z \circ \Phi_{*}^{-1}, \Phi(\mathcal{A})\right)$, where $\Phi \in \operatorname{Aut}\left(\mathrm{D}^{\mathrm{b}}(X)\right)$ and $\Phi_{*}$ is the automorphism induced by $\Phi$ at the level of numerical Grothendieck groups. We will often abuse notation and denote $\Phi_{*}$ by $\Phi$, when no confusion arises. The universal cover $\widetilde{G L}_{2}^{+}(\mathbb{R})$ of the group $\mathrm{GL}_{2}^{+}(\mathbb{R})$ of matrices with positive determinant acts on the right as a lift of the action of $\mathrm{GL}_{2}^{+}(\mathbb{R})$ on $\operatorname{Hom}\left(K_{\text {num }}(X), \mathbb{C}\right) \cong$ $\operatorname{Hom}\left(K_{\text {num }}(X), \mathbb{R}^{2}\right)$. We typically only use the action of the subgroup $\mathbb{C} \subset \widetilde{\mathrm{GL}}_{2}^{+}(\mathbb{R})$ given 
PROJECTIVITY AND BIRATIONAL GEOMETRY OF BRIDGELAND MODULI SPACES

as the universal cover of $\mathbb{C}^{*} \subset \mathrm{GL}_{2}^{+}(\mathbb{R})$ : given $z \in \mathbb{C}$, it acts on $(Z, \mathcal{A})$ by $Z \mapsto e^{2 \pi i z} \cdot Z$, and by modifying $\mathcal{A}$ accordingly.

\section{Positivity}

In this section we prove our main result, Positivity Lemma 3.3.

We consider any smooth projective variety $X$ with a numerical stability condition $\sigma=$ $(Z, \mathcal{A})$ on $\mathrm{D}^{\mathrm{b}}(X)$. Let us first recall the definition of flat families, due to Bridgeland:

Definition 3.1. Let $\mathcal{A} \subset \mathrm{D}^{\mathrm{b}}(X)$ be the heart of a bounded t-structure on $\mathrm{D}^{\mathrm{b}}(X)$. Let $S$ be an algebraic space of finite type over $\mathbb{C}$, and let $\mathcal{E} \in \mathrm{D}_{S \text {-perf }}(S \times X)$. We say that $\mathcal{E}$ is flat with respect to $\mathcal{A}$ if, for every closed point $s \in S$, the derived restriction $\mathcal{E}_{s}$ belongs $\mathcal{A}$.

Let $v \in K_{\text {num }}(X)$; we use the action of $\mathbb{C}$ on $\operatorname{Stab}(X)$ described in Remark 2.6 to assume $Z(v)=-1$. We denote by $\mathfrak{M}_{\sigma}(v)$ be the moduli stack of flat families of $\sigma$-semistable objects of class $v$ and phase 1. Our construction in this section gives a version of Theorem 1.1 for the stack $\mathfrak{M}_{\sigma}(v)$; we will discuss how it extends to the coarse moduli space (when it exists) in Section 4.

Proposition and Definition 3.2. Let $C \rightarrow \mathfrak{M}_{\sigma}(v)$ be an integral projective curve over $\mathfrak{M}_{\sigma}(v)$, with induced universal family $\mathcal{E} \in \mathrm{D}^{\mathrm{b}}(C \times X)$, and associated Fourier-Mukai transform $\Phi_{\mathcal{E}}: \mathrm{D}^{\mathrm{b}}(C) \rightarrow \mathrm{D}^{\mathrm{b}}(X)$. To such a $C$ we associate a number $\mathfrak{L}_{\sigma} . C \in \mathbb{R}$ by

$$
\mathfrak{L}_{\sigma} \cdot C:=\Im Z\left(\Phi_{\mathcal{E}}\left(\mathcal{O}_{C}\right)\right) .
$$

This has the following properties:

(a) Modifying the universal family by tensoring with a pull-back of a line bundle on $C$ does not modify $\mathfrak{L}_{\sigma} . C$.

(b) We can replace $\mathcal{O}_{C}$ in equation (4) by any line bundle on $C$, without changing $\mathfrak{L}_{\sigma} . C$.

We will think of $\mathfrak{L}_{\sigma}$ as a divisor class in $N^{1}\left(\mathfrak{M}_{\sigma}(v)\right)$.

Proof. If $c \in C$, then replacing the universal family by $\mathcal{E}^{\prime}=\mathcal{E} \otimes p^{*} \mathcal{O}_{C}(c)$ effects the Fourier-Mukai transform by

$$
\left[\Phi_{\mathcal{E}^{\prime}}\left(\mathcal{O}_{C}\right)\right]=\left[\Phi_{\mathcal{E}}\left(\mathcal{O}_{C}\right)\right]+\left[\Phi_{\mathcal{E}}(k(c))\right]=\left[\Phi_{\mathcal{E}}\left(\mathcal{O}_{C}\right)\right]+v
$$

As $\Im Z(v)=0$, this proves claim (a), and similarly (b).

Positivity Lemma 3.3. The divisor class $\mathfrak{L}_{\sigma}$ is nef: $\mathfrak{L}_{\sigma} . C \geq 0$. Further, we have $\mathfrak{L}_{\sigma} . C>$ 0 if and only if for two general closed points $c, c^{\prime} \in C$, the corresponding objects $\mathcal{E}_{c}, \mathcal{E}_{c^{\prime}} \in$ $\mathrm{D}^{\mathrm{b}}(X)$ are not $S$-equivalent.

We first point out that by Lemma 2.5, we can immediately restrict to the case where $\sigma$ is an algebraic stability condition. This implies that the heart $\mathcal{A}$ is Noetherian, by [AP06, Proposition 5.0.1].

The essential ingredient in the proof is the construction and description by Abramovich and Polishchuk of a constant family of t-structures on $S \times X$ induced by $\mathcal{A}$, for smooth $S$ given in [AP06] and extended to singular $S$ in [Pol07]. For any scheme $S$ of finite type over 
$\mathbb{C}$, we denote by $\mathcal{A}_{S}$ the heart of the "constant t-structure" on $\mathrm{D}^{\mathrm{b}}(S \times X)$ given by [Pol07, Theorem 3.3.6]. The heart $\mathcal{A}_{S}$ could be thought of as Coh $S \otimes \mathcal{A}$, since it behaves like $\mathcal{A}$ with respect to $X$, and like Coh $S$ with respect to $S$. For example, whenever $F \in \operatorname{Coh} S$ and $E \in \mathcal{A}$, we have $F \otimes E \in \mathcal{A}_{S}$; also, $\mathcal{A}_{S}$ is invariant under tensoring with line bundles pulled back from $S$. It is characterized by the following statements (which paraphrase [Pol07, Theorem 3.3.6]):

Theorem 3.4. Let $\mathcal{A}$ be the heart of a Noetherian bounded $t$-structure on $\mathrm{D}^{\mathrm{b}}(X)$. Denote by $\mathcal{A}^{q c} \subset \mathrm{D}_{q c}(X)$ the closure of $\mathcal{A}$ under infinite coproducts in the derived category of quasi-coherent sheaves.

(a) For any scheme $S$ of finite type of $\mathbb{C}$ there is a Noetherian bounded t-structure on $\mathrm{D}^{\mathrm{b}}(S \times X)$, whose heart $\mathcal{A}_{S}$ is characterized by

$$
\left.\mathcal{E} \in \mathcal{A}_{S} \Leftrightarrow p_{*} \mathcal{E}\right|_{X \times U} \in \mathcal{A}^{q c} \quad \text { for every open affine } U \subset S
$$

(b) The above construction defines a sheaf of t-structures over $S$ : when $S=\bigcup_{i} U_{i}$ is an open covering of $S$, then $\mathcal{E} \in \mathcal{A}_{S}$ if and only if $\left.\mathcal{E}\right|_{X \times U_{i}} \in \mathcal{A}_{U_{i}}$ for every $i$.

(c) When $S$ is projective and $\mathcal{O}_{S}(1)$ denotes an ample divisor, then

$$
\mathcal{E} \in \mathcal{A}_{S} \Leftrightarrow\left(p_{X}\right)_{*}\left(\mathcal{E} \otimes p_{S}^{*} \mathcal{O}_{S}(n)\right) \in \mathcal{A} \quad \text { for all } n \gg 0 .
$$

The following lemma is essentially [Pol07, Proposition 2.3.7] (see also [AP06, Corollary 3.3.3] for the smooth case):

Lemma 3.5. Let $\mathcal{E} \in \mathrm{D}^{\mathrm{b}}(S \times X)$ be a flat family of objects in $\mathcal{A}$. Then $\mathcal{E} \in \mathcal{A}_{S}$.

Proof. We first claim the statement when $S$ is a zero-dimensional scheme of finite length $l>0$, with a unique closed point $s \in S$. Choose a filtration

$$
0=F_{0} \subset F_{1} \subset \ldots \subset F_{l}=\mathcal{O}_{S},
$$

of the structure sheaf in Coh $S$ with $F_{i} / F_{i-1} \cong k(s)$ for all $i$. After pull-back to $S \times X$ and tensoring with $\mathcal{E}$ we get a sequence of morphisms in $\mathrm{D}^{\mathrm{b}}(S \times X)$

$$
0=G_{0} \rightarrow G_{1} \rightarrow \ldots \rightarrow G_{l}=\mathcal{E}
$$

such that cone $\left(G_{i-1} \rightarrow G_{i}\right) \cong \mathcal{E}_{s}$ for all $i$. By induction on $i$ we obtain $\left(p_{X}\right)_{*} G_{i} \in \mathcal{A}$; then part (c) of Theorem 3.4 implies $\mathcal{E} \in \mathcal{A}_{S}$.

For general $S$, any closed point $s \in S$ is contained in a local zero-dimensional subscheme $T \subset S$ that is a local complete intersection in $S$. The previous case shows $\mathcal{E}_{T} \in \mathcal{A}_{T}$, and by [Pol07, Proposition 2.3.7] we can cover $S$ by open sets $U$ with $\mathcal{E}_{U} \in \mathcal{A}_{U}$. By the sheaf property (b), this shows $\mathcal{E} \in \mathcal{A}_{S}$.

Lemma 3.6. Let $C$ be an integral projective curve, and $\mathcal{E} \in D^{b}(C \times X)$ be a family of $\sigma$-semistable objects in $\mathcal{P}(1)$. Then, there exists $n_{0}>0$ such that

$$
\Phi_{\mathcal{E}}(L) \in \mathcal{A}
$$

for all line bundles $L$ on $C$ with degree $\operatorname{deg}(L) \geq n_{0}$. 
Proof. By the previous lemma, we have $\mathcal{E} \in \mathcal{A}_{C}$. Fix an ample divisor $\mathcal{O}_{C}(1)$ on $C$. By the statement (c) of Theorem 3.4, there exists $m_{0}>0$ such that

$$
\Phi_{\mathcal{E}}\left(\mathcal{O}_{C}(n)\right)=\left(p_{X}\right)_{*}\left(\mathcal{E} \otimes p_{C}^{*} \mathcal{O}_{C}(n)\right) \in \mathcal{A},
$$

for all $n \geq m_{0}$. Fix $n_{0}>0$ such that, for a line bundle $L$ with $\operatorname{deg}(L) \geq n_{0}$, we have $H^{0}\left(C, L\left(-m_{0}\right)\right) \neq 0$. Then, consider the exact sequence

$$
0 \rightarrow \mathcal{O}\left(m_{0}\right) \rightarrow L \rightarrow T \rightarrow 0
$$

where $T$ has zero-dimensional support. By applying $\Phi_{\mathcal{E}}$ we get our claim.

Lemma 3.6 directly implies the first claim of Positivity Lemma 3.3: For a curve $C \rightarrow$ $\mathfrak{M}_{\sigma}(v)$ with universal family $\mathcal{E} \in \mathrm{D}^{\mathrm{b}}(X \times C)$, we have

$$
\mathfrak{L}_{\sigma} \cdot C=\Im Z\left(\Phi_{\mathcal{E}}\left(\mathcal{O}_{C}\right)\right)=\Im Z\left(\Phi_{\mathcal{E}}\left(\mathcal{O}_{C}(n)\right)\right) \geq 0,
$$

by the basic positivity property of the central charge $Z$ in equation (3).

It remains to prove the second claim.

Lemma 3.7. Let $\mathcal{E} \in \mathrm{D}^{\mathrm{b}}(S \times X)$ be a flat family of semistable objects in $\mathcal{A}$ over an irreducible scheme $S$ of finite type over $\mathbb{C}$. Assume that the union of all Jordan-Hölder factors of $\mathcal{E}_{s}$ over all closed points $s \in S$ is finite. Then all the objects $\mathcal{E}_{s}$ are $S$-equivalent to each other, and we can choose a Jordan-Hölder filtration for every $\mathcal{E}_{s}$ such that the order of their stable filtration quotients does not depend on $s$.

Proof. If we choose a Jordan-Hölder filtration of $\mathcal{E}_{s}$ for every closed point $s$, then there will be a stable object $F \in \mathcal{A}$ that appears as the final quotient $\mathcal{E}_{s} \rightarrow F$ of the filtration for infinitely many $s \in S$. In particular, $\operatorname{Hom}\left(\mathcal{E}_{s}, F\right)$ is non-zero for infinitely many $s \in S$; by semi-continuity, this implies that for every $s \in S$ there is a (necessarily surjective) morphism $\mathcal{E}_{s} \rightarrow F$ in $\mathcal{A}$. The same argument applied to the kernel of $\mathcal{E}_{s} \rightarrow F$ implies the claim by induction on the length of $\mathcal{E}_{s_{0}}$ for a fixed chosen point $s_{0} \in S$.

Lemma 3.8. Let $F \in \mathcal{A}$ be a simple object. Then any subobject of $p_{X}^{*} F$ in $\mathcal{A}_{S}$ is of the form $I \otimes F$ for some ideal sheaf $I \subset \mathcal{O}_{S}$ on $S$.

Proof. By the sheaf property of $\mathcal{A}_{S}$, it is sufficient to treat the case where $S$ is affine. By the characterization (a) in Theorem 3.4 of $\mathcal{A}_{S}$, a subobject $G \subset p_{X}^{*} F$ in $\mathcal{A}_{S}$ gives a subobject $\left(p_{X}\right)_{*} G$ of $\left(p_{X}\right)_{*} p_{X}^{*} F=\mathcal{O}_{S} \otimes_{\mathbb{C}} F$ in $\mathcal{A}^{q c}$ that is compatible with the $\mathcal{O}_{S}$-module structure (see also [Pol07, Proposition 3.3.7]). Since $F$ is simple, such a subobject must be of the form $I \otimes_{\mathbb{C}} F$ for some ideal $I \subset \mathcal{O}_{S}$.

Lemma 3.9. Let $\mathcal{E} \in \mathrm{D}^{\mathrm{b}}(C \times X)$ be a family of semistable objects over an integral curve $C$. Assume that for general $c, c^{\prime} \in C$, the objects $\mathcal{E}_{c}, \mathcal{E}_{c^{\prime}}$ are $S$-equivalent to each other. Then there exist line bundles $\mathcal{L}_{1}, \ldots, \mathcal{L}_{n}$ on $C$ and a filtration

$$
0=\Gamma_{0} \subset \Gamma_{1} \subset \ldots \subset \Gamma_{n}=\mathcal{E}
$$

in $\mathcal{A}_{S}$ such that, for all $i=1, \ldots, n$,

$$
\Gamma_{i} / \Gamma_{i-1} \cong F_{i} \otimes \mathcal{L}_{i}
$$


and such the restrictions of the $\Gamma_{i}$ to the fibers $\{c\} \times X$ induces the Jordan-Hölder filtration of $\mathcal{E}_{c}$ for all but finitely many $c \in C$.

Proof. The same arguments as in the previous lemma show that any two $\mathcal{E}_{c}, \mathcal{E}_{c^{\prime}}$ are $S$-equivalent to each other, and that there is a common stable subobject $F:=F_{1} \subset \mathcal{E}_{s}$ for all $s$.

We first claim that there exists a line bundle $\mathcal{L}_{1}$ on $C$ with a non-zero morphism $\phi: F \otimes$ $\mathcal{L}_{1} \rightarrow \mathcal{E}$ on $C \times X$; equivalently, we need to show that for $\mathcal{L}:=\mathcal{L}_{1}^{*}$, we have

$$
0 \neq \operatorname{Hom}_{X}\left(F,\left(p_{X}\right)_{*}\left(\mathcal{E} \otimes p_{C}^{*} \mathcal{L}\right)\right)=\operatorname{Hom}_{X}\left(F, \Phi_{\mathcal{E}}(\mathcal{L})\right) .
$$

Let $n_{0}$ be as in Lemma 3.6, and fix a line bundle $\mathcal{L}_{0}$ on $C$ of degree $n_{0}$. Set $r:=$ $\operatorname{dim} \operatorname{Ext}^{1}\left(F, \Phi_{\mathcal{E}}\left(\mathcal{L}_{0}\right)\right)$. Pick $r+1$ distinct smooth points $c_{1}, \ldots, c_{r+1} \in C$, and set $\mathcal{L}:=$ $\mathcal{L}_{0}\left(c_{1}+\cdots+c_{r+1}\right)$. Consider the short exact sequence

$$
0 \rightarrow \mathcal{L}_{0} \rightarrow \mathcal{L} \rightarrow \mathcal{O}_{c_{1}} \oplus \cdots \oplus \mathcal{O}_{c_{r+1}} \rightarrow 0
$$

in Coh $C$. By Lemma 3.6, it induces a short sequence

$$
0 \rightarrow \Phi_{\mathcal{E}}\left(\mathcal{L}_{0}\right) \rightarrow \Phi_{\mathcal{E}}(\mathcal{L}) \rightarrow \mathcal{E}_{c_{1}} \oplus \cdots \oplus \mathcal{E}_{c_{r+1}} \rightarrow 0
$$

in $\mathcal{A}$. Since $\operatorname{dim} \operatorname{Hom}\left(F, \mathcal{E}_{c_{1}} \oplus \cdots \oplus \mathcal{E}_{c_{r+1}}\right) \geq r+1>r=\operatorname{dim} \operatorname{Ext}^{1}\left(F, \Phi_{\mathcal{E}}\left(\mathcal{L}_{0}\right)\right)$, there exists a non-zero morphism from $F$ to the direct sum that factors via $\Phi_{\mathcal{E}}(\mathcal{L})$, which proves the existence of the morphism $\phi$ as claimed.

It follows from [Lie06, Proposition 2.2.3] that the restriction $\phi_{c}: F_{1} \rightarrow \mathcal{E}_{c}$ is non-zero for all but finitely many closed points $c \in C$. Since $F_{1}$ is stable, this morphism is necessarily injective. To proceed by induction, it remains to show that $\phi$ is an injective morphism in $\mathcal{A}_{C}$. Otherwise, by Lemma 3.8 the kernel of $\phi$ is of the form $I \otimes \mathcal{L}_{1} \otimes F_{1}$ for some ideal sheaf $I \subset \mathcal{O}_{C}$. In particular, the derived restriction of $\operatorname{ker} \phi \hookrightarrow \mathcal{L}_{1} \otimes F_{1}$ to $\{c\} \times X$ is an isomorphism for all but finitely many $c$, in contradiction to the injectivity of $\phi_{c}$.

Proof. (Positivity Lemma 3.3) Let $C$ be an integral projective curve, and let $\mathcal{E} \in$ $\mathfrak{M}_{\sigma}(v)(C)$. As we observed before, we only need to prove the second claim.

"६": Assume that $\mathfrak{L}_{\sigma} . C=0$. We will show that all objects $\mathcal{E}_{c}$, for smooth points $c \in C$, are $S$-equivalent to each other.

By Lemma 3.6, for a line bundle $L$ of large degree on $C$, and for $c \in C$ a smooth point, the short exact sequence

$$
0 \rightarrow L(-c) \rightarrow L \rightarrow k(c) \rightarrow 0
$$

induces a short exact sequence in $\mathcal{A}$

$$
0 \rightarrow \Phi_{\mathcal{E}}(L(-c)) \rightarrow \Phi_{\mathcal{E}}(L) \rightarrow \mathcal{E}_{c} \rightarrow 0 .
$$

Since $\mathfrak{L}_{\sigma} . C=0$, we have $Z\left(\Phi_{\mathcal{E}}(L)\right) \in \mathbb{R}_{<0}$, and so $\Phi_{\mathcal{E}}(L) \in \mathcal{P}(1)$ is semistable, of the same phase as $\mathcal{E}_{c}$. It follows that the Jordan-Hölder factors of $\mathcal{E}_{c}$ are a subset of the JordanHölder factors of $\Phi_{\mathcal{E}}(L)$, which of course do not depend on $c$. Lemma 3.7 implies the claim. 
" $\Rightarrow$ ": Assume that, for general $c \in C$, all objects $\mathcal{E}_{c}$ are $S$-equivalent to each other. Using Lemma 3.9 and the projection formula, we obtain:

$$
\begin{aligned}
\mathfrak{L}_{\sigma} . C & =\Im Z\left(\left[\Phi_{\mathcal{E}}\left(\mathcal{O}_{C}\right)\right]\right)=\sum_{i=1}^{n} \Im Z\left(\left[\left(p_{X}\right)_{*} F_{i} \otimes \mathcal{L}_{i}\right]\right) \\
& =\sum_{i=1}^{n} \Im Z\left(\left[F_{i} \otimes H^{\bullet}\left(C, \mathcal{L}_{i}\right)\right]\right)=\sum_{i=1}^{n} \chi\left(C, \mathcal{L}_{i}\right) \cdot \Im Z\left(\left[F_{i}\right]\right)=0
\end{aligned}
$$

\section{A natural NeF Divisor Class on the MOdUli SPACE, AND COMPARison}

Let $S$ be a proper algebraic space of finite type over $\mathbb{C}$, let $\sigma=(Z, \mathcal{A}) \in \operatorname{Stab}(X)$, and let $\mathcal{E} \in \mathrm{D}_{S \text {-perf }}(S \times X)$ be a flat family of semistable objects of class $v$ with $Z(v)=-1$. Note that $S$ is allowed to have arbitrary singularities.

By restriction of the family, our construction in the previous section assigns a number $\mathfrak{L}_{\sigma} . C$ to every curve $C \subset S$. Our first goal is to prove that this induces a nef divisor class on $S$, as claimed in Theorem 1.1; the following theorem gives a more complete statement:

Theorem 4.1. The assignment $C \mapsto \mathfrak{L}_{\sigma} . C$ only depends on the numerical curve class $[C] \in N_{1}(S)$, and is additive on curve classes. It defines a nef divisor class $\ell_{\sigma, \mathcal{E}} \in N^{1}(S)$, which is invariant under tensoring the family $\mathcal{E}$ with a line bundle pulled back from $S$. Additionally, for a curve $C \subseteq S$ we have $\ell_{\sigma, \mathcal{E}} \cdot C>0$ if and only if for two general closed points $c, c^{\prime} \in C$, the corresponding objects $\mathcal{E}_{c}, \mathcal{E}_{c^{\prime}} \in \mathrm{D}^{\mathrm{b}}(X)$ are not $S$-equivalent.

Before the proof, let us recall that the real Néron-Severi group $N^{1}(S)$ is defined as the group of real Cartier divisors modulo numerical equivalence; dually, $N_{1}(S)$ is the group of real 1-cycles modulo numerical equivalence with respect to pairing with Cartier divisors (see, for example, [Laz04, Sections 1.3 and 1.4]).

Similarly, the Euler characteristic gives a well-defined pairing

$$
\chi: K\left(\mathrm{D}^{\mathrm{b}}(S)\right) \times K\left(\mathrm{D}_{\text {perf }}^{\mathrm{b}}(S)\right) \rightarrow \mathbb{Z}
$$

between the K-groups of the bounded derived categories of coherent sheaves $\mathrm{D}^{\mathrm{b}}(S)$ and of perfect complexes $\mathrm{D}_{\text {perf }}^{\mathrm{b}}(S)$. Taking the quotient with respect to the kernel of $\chi$ on each side we obtain numerical Grothendieck groups $K_{\text {num }}(S)$ and $K_{\text {num }}^{\text {perf }}(S)$, respectively, with an induced perfect pairing

$$
\chi: K_{\text {num }}(S) \times K_{\text {num }}^{\text {perf }}(S) \rightarrow \mathbb{Z} .
$$

Proof of Theorem 4.1. If $C_{1}, C_{2}$ are numerically equivalent, then $\left[\mathcal{O}_{C_{1}}\right],\left[\mathcal{O}_{C_{2}}\right] \in K_{\text {num }}(S)$ only differ by multiples of the class of a skyscraper sheaf $k(s)$ of a closed point $s$. Since $\mathcal{E}$ is $S$-perfect, it induces a functor $\Phi_{\mathcal{E}}: \mathrm{D}^{\mathrm{b}}(S) \rightarrow \mathrm{D}^{\mathrm{b}}(X)$, and this functor preserves numerical equivalence. Together with $\Im Z\left(\Phi_{\mathcal{E}}(k(s))\right)=\Im Z(v)=0$, this proves the first claim. The additivity follows similarly, and all other claims follow directly from the Positivity Lemma 3.3 . 
Example 4.2. Let $X \subset \mathbb{P}^{4}$ be a smooth quintic threefold, containing two disjoint lines $L_{1}, L_{2}$. Consider the smooth proper algebraic space $X^{+}$obtained as the flop of $X$ at the line $L_{1}$. Then, by a classical result of Bondal and Orlov [BO95], we have an equivalence of derived categories $\mathrm{D}^{\mathrm{b}}(X) \cong \mathrm{D}^{\mathrm{b}}\left(X^{+}\right)$. However, $X^{+}$admits no numerically positive class, as the flopped curve $\widetilde{L}_{1}$ is the negative of $L_{2}$ in $N_{1}\left(X^{+}\right)$. By Theorem $1.1, X^{+}$cannot be isomorphic to a moduli space of (semi)stable complexes on $\mathrm{D}^{\mathrm{b}}(X)$ with respect to a numerical stability condition on $X$.

There are many examples of non-projective flops of a projective variety, including Mukai flops of holomorphic symplectic varieties. By the same reasoning, these non-projective flops cannot be obtained by wall-crossing.

We will now compare our construction to the classical notion of a determinant divisor on $S$ associated to the family $\mathcal{E}$ (see, e.g., [Muk87, Don90, LP92, Fal93, Li96] and [HL10, Section 8.1]).

Definition 4.3. We define a group homomorphism (called the Donaldson morphism $)^{2}$

$$
\lambda_{\mathcal{E}}: v^{\sharp} \rightarrow N^{1}(S)
$$

as the composition

$$
v^{\sharp} \stackrel{p_{X}^{*}}{\longrightarrow} K_{\text {num }}^{\text {perf }}(S \times X)_{\mathbb{R}} \stackrel{\cdot[\mathcal{E}]}{\longrightarrow} K_{\text {num }}^{\text {perf }}(S \times X)_{\mathbb{R}} \stackrel{\left(p_{S}\right)_{*}}{\longrightarrow} K_{\text {num }}^{\text {perf }}(S)_{\mathbb{R}} \stackrel{\operatorname{det}}{\longrightarrow} N^{1}(S),
$$

where

$$
v^{\sharp}:=\left\{w \in K_{\text {num }}(X)_{\mathbb{R}}: \chi(v \cdot w)=0\right\} .
$$

Since the Euler characteristic $\chi$ gives a non-degenerate pairing, we can write

$$
\Im\left(Z\left(\_\right)\right)=\chi\left(w_{Z} \cdot \_\right)
$$

for a unique vector $w_{Z} \in v^{\sharp}$.

Proposition 4.4. For an integral curve $C \subset S$, we have

$$
\lambda_{\mathcal{E}}\left(w_{Z}\right) \cdot C=\Im Z\left(\Phi_{\mathcal{E}}\left(\mathcal{O}_{C}\right)\right)=: \ell_{\sigma, \mathcal{E}} \cdot C .
$$

Proof. It is enough to prove (5) when $w_{Z}=[F] \in K_{\text {num }}(X)$, for some $F \in \mathrm{D}^{\mathrm{b}}(X)$. We define

$$
\mathcal{L}(F):=\left(p_{S}\right)_{*}\left(p_{X}^{*} F \otimes \mathcal{E}\right) .
$$

As in the classical case, the assumption $w_{Z} \in v^{\sharp}$ and the projection formula show that the rank of $\mathcal{L}(F)$ must be equal to 0 :

$\operatorname{rk} \mathcal{L}(F)=\chi\left(S, \mathcal{O}_{s} \otimes \mathcal{L}(F)\right)=\chi\left(S \times X, \mathcal{O}_{\{s\} \times X} \otimes \mathcal{E} \otimes p_{X}^{*} F\right)=\chi\left(\mathcal{E}_{s} \otimes F\right)=\chi\left(v \cdot w_{Z}\right)=0$.

The Riemann-Roch Theorem gives

$$
\lambda_{\mathcal{E}}\left(w_{Z}\right) \cdot C=\left.\operatorname{deg} \mathcal{L}(F)\right|_{C}=\chi\left(C,\left.\mathcal{L}(F)\right|_{C}\right) .
$$

By Cohomology and Base Change (see, e.g., [Kuz06, Corollary 2.23]) we deduce that

$$
\left.\mathcal{L}(F)\right|_{C}=\left(p_{C}\right)_{*}\left(\left.p_{X}^{*} F \otimes \mathcal{E}\right|_{C}\right) .
$$

\footnotetext{
${ }^{2}$ In the case of K3 surfaces, we will instead use a dual version, see Definition 5.4 and Remark 5.5.
} 
Using the projection formula again gives

$$
\chi\left(C,\left.\mathcal{L}(F)\right|_{C}\right)=\chi\left(S,\left.F \otimes\left(p_{X}\right)_{*} \mathcal{E}\right|_{C}\right)=\chi\left(w_{Z} \cdot \Phi_{\mathcal{E}}\left(\mathcal{O}_{C}\right)\right)=\Im Z\left(\Phi_{\mathcal{E}}\left(\mathcal{O}_{C}\right)\right) .
$$

The basic properties of $\ell_{\sigma, \mathcal{E}}$ are in [HL10, Lemma 8.1.2]. In particular, we recall the following: Let $\mathcal{N}$ be a vector bundle on $S$ of rank $n$. Then

$$
\ell_{\sigma, \mathcal{E} \otimes p_{S}^{*} \mathcal{N}}=n \cdot \ell_{\sigma, \mathcal{E}}
$$

By Theorem 4.1, we have a well-defined positive divisor class on a fine moduli space of stable complexes. To extend this to the case when a universal family exists only étale locally on a coarse moduli space, we recall the following definition from [Muk87].

Definition 4.5. Let $T$ be an algebraic space of finite-type over $\mathbb{C}$.

(a) A flat family $\mathcal{E}$ on $T \times X$ is called a quasi-family of objects in $\mathfrak{M}_{\sigma}(v)$ if, for all closed points $t \in T$, there exist an integer $\rho>0$ and an element $E \in \mathfrak{M}_{\sigma}(v)(\mathbb{C})$ such that $\left.\mathcal{E}\right|_{\{t\} \times X} \cong E^{\oplus \rho}$. If $T$ is connected, the positive integer $\rho$ does not depend on $t$ and it is called the similitude of $\mathcal{E}$.

(b) Two quasi-families $\mathcal{E}$ and $\mathcal{E}^{\prime}$ on $T \times X$ are called equivalent if there exist vector bundles $V$ and $V^{\prime}$ on $T$ such that $\mathcal{E}^{\prime} \otimes p_{T}^{*} V \cong \mathcal{E} \otimes p_{T}^{*} V^{\prime}$.

(c) A quasi-family $\mathcal{E}$ is called quasi-universal if, for every scheme $T^{\prime}$ and for any quasifamily $\mathcal{T}$ on $T \times X$, there exists a unique morphism $f: T^{\prime} \rightarrow T$ such that $f^{*} \mathcal{E}$ and $\mathcal{T}$ are equivalent.

Let $\mathcal{E}$ be a quasi-family of objects in $\mathfrak{M}_{\sigma}(v)$ of similitude $\rho$ over a proper algebraic space

$T$. We can define a divisor class $\ell_{\sigma}$ on $T$ by $\ell_{\sigma}:=\frac{1}{\rho} \cdot \ell_{\sigma, \mathcal{E}}$; by equation (6) it only depends on the equivalence class of the quasi-universal family.

Remark 4.6. By [Muk87, Theorem A.5], if $\mathfrak{M}_{\sigma}(v)$ consists only of stable (and therefore, simple) complexes, and if $\mathfrak{M}_{\sigma}(v)$ is a $\mathbb{G}_{m}$-gerbe over an algebraic space $M_{\sigma}(v)$ of finite-type over $\mathbb{C}$ (i.e., over its coarse moduli space), then there exists a quasi-universal family on $M_{\sigma}(v) \times X$, unique up to equivalence.

Therefore, the above construction produces a well-defined divisor class $\ell_{\sigma}$ on the coarse moduli space $M_{\sigma}(v)$. Theorem 4.1 holds similarly for $\ell_{\sigma}$; in particular, it has the same positivity properties.

\section{Review: Moduli spaces for stable sheaves on K3 surfaces}

In this section we give a summary on stability for sheaves on K3 surfaces. We start by recalling the basic lattice-theoretical structure, given by the Mukai lattice. We then review slope and Gieseker stability, the existence and non-emptiness for moduli spaces of semistable sheaves, and the structure of their Néron-Severi groups. Finally, we mention briefly how all of this generalizes to twisted K3 surfaces. 
The algebraic Mukai lattice. Let $X$ be a smooth projective K3 surface. We denote by $H_{\text {alg }}^{*}(X, \mathbb{Z})$ the algebraic part of the whole cohomology of $\mathrm{X}$, namely

$$
H_{\mathrm{alg}}^{*}(X, \mathbb{Z})=H^{0}(X, \mathbb{Z}) \oplus \mathrm{NS}(X) \oplus H^{4}(X, \mathbb{Z}) .
$$

Let $v: K_{\text {num }}(X) \stackrel{\sim}{\rightarrow} H_{\text {alg }}^{*}(X, \mathbb{Z})$ be the Mukai vector given by $v(E)=\operatorname{ch}(E) \sqrt{\operatorname{td}(X)}$. We denote the Mukai pairing $H_{\text {alg }}^{*}(X, \mathbb{Z}) \times H_{\text {alg }}^{*}(X, \mathbb{Z}) \rightarrow \mathbb{Z}$ by (_,_); it can be defined by $(v(E), v(F)):=-\chi(E, F)$. According to the decomposition (7), we have

$$
\left((r, c, s),\left(r^{\prime}, c^{\prime}, s^{\prime}\right)\right)=c . c^{\prime}-r s^{\prime}-r^{\prime} s,
$$

for $(r, c, s),\left(r^{\prime}, c^{\prime}, s^{\prime}\right) \in H_{\text {alg }}^{*}(X, \mathbb{Z})$.

Given a Mukai vector $v \in H_{\mathrm{alg}}^{*}(X, \mathbb{Z})$, we denote its orthogonal complement by

$$
v^{\perp}:=\left\{w \in H_{\text {alg }}^{*}(X, \mathbb{Z}):(v, w)=0\right\} .
$$

We call a Mukai vector $v$ primitive if it is not divisible in $H_{\mathrm{alg}}^{*}(X, \mathbb{Z})$.

Slope stability. Let $\omega, \beta \in \mathrm{NS}(X)_{\mathbb{Q}}$ with $\omega$ ample. We define a slope function $\mu_{\omega, \beta}$ on Coh $X$ by

$$
\mu_{\omega, \beta}(E)= \begin{cases}\frac{\omega \cdot\left(c_{1}(E)-\beta\right)}{r(E)} & \text { if } r(E)>0, \\ +\infty & \text { if } r(E)=0 .\end{cases}
$$

This gives a notion of slope stability for sheaves, for which Harder-Narasimhan filtrations exist (see [HL10, Section 1.6]). We will sometimes use the notation $\mu_{\omega, \beta}$-stability.

Set-theoretical moduli spaces of torsion-free slope semistable sheaves were constructed by Le Potier and Li (see [LP92, Li93] and [HL10, Section 8.2]).

Gieseker stability. Let $\omega, \beta \in \mathrm{NS}(X)_{\mathbb{Q}}$ with $\omega$ ample. We define the twisted Hilbert polynomial by

$$
P(E, m):=\int_{X} e^{-\beta} \cdot\left(1, m \omega, \frac{m^{2} \omega^{2}}{2}\right) \cdot v(E),
$$

for $E \in \operatorname{Coh}(X)$. This gives rise to the notion of $\beta$-twisted $\omega$-Gieseker stability for sheaves, introduced first in [MW97]. When $\beta=0$, this is nothing but Gieseker stability. We refer to [HL10, Section 1] for basic properties of Gieseker stability.

Moduli spaces of stable sheaves. Let $\omega, \beta \in \mathrm{NS}(X)_{\mathbb{Q}}$ with $\omega$ ample. We fix a Mukai vector $v \in H_{\text {alg }}^{*}(X, \mathbb{Z})$. We denote by $\mathfrak{M}_{\omega}^{\beta}(v)$ the moduli stack of flat families of $\beta$-twisted $\omega$-Gieseker semistable sheaves with Mukai vector $v$. By the work of Mumford, Gieseker, Maruyama, and Simpson among others (see [HL10, Section 4] and [MW97]), there exists a projective variety $M_{\omega}^{\beta}(v)$ which is a coarse moduli space parameterizing $S$-equivalence classes of semistable sheaves. The open substack $\mathfrak{M}_{\omega}^{\beta, s}(v) \subseteq \mathfrak{M}_{\omega}^{\beta}(v)$ parameterizing stable sheaves is a $\mathbb{G}_{m}$-gerbe over the open subset $M_{\omega}^{\beta, s}(v) \subseteq M_{\omega}^{\beta}(v)$. When $\beta=0$, we will denote the corresponding objects by $\mathfrak{M}_{\omega}(v)$, etc.

The following is the main result on moduli spaces of stable sheaves on K3 surfaces. In its final form it is proved by Yoshioka in [Yos01b, Theorems 0.1 \& 8.1] (see also [KLS06, 
Section 2.4], where the condition of positivity is discussed more in detail); it builds on previous work by Mukai and Kuleshov, among others. We start by recalling the notion of positive vector, following [Yos01b, Definition 0.1].

Definition 5.1. Let $v_{0}=(r, c, s) \in H_{\text {alg }}^{*}(X, \mathbb{Z})$ be a primitive class. We say that $v_{0}$ is positive if $v_{0}^{2} \geq-2$ and

- either $r>0$,

- or $r=0, c$ is effective, and $s \neq 0$,

- or $r=c=0$ and $s>0$.

Theorem 5.2 (Yoshioka). Let $v \in H_{\mathrm{alg}}^{*}(X, \mathbb{Z})$. Assume that $v=m v_{0}$, with $m \in \mathbb{Z}_{>0}$ and $v_{0}$ a primitive positive vector. Then $M_{\omega}^{\beta}(v)$ is non-empty for all $\omega, \beta$.

Remark 5.3. We keep the assumptions of Theorem 5.2. We further assume that $\omega$ is generic ${ }^{3}$ with respect to $v$.

(a) By [O'G99, KLS06, PR11], $M_{\omega}^{\beta}(v)$ is then a normal irreducible projective variety with $\mathbb{Q}$-factorial singularities.

(b) If $m=1$, then by [Yos01b], based on previous work by Mukai, O'Grady, and Huybrechts among others, $M_{\omega}^{\beta, s}(v)=M_{\omega}^{\beta}(v)$ is a smooth projective irreducible symplectic manifold of dimension $v^{2}+2$, deformation equivalent to the Hilbert scheme of points on a K3 surface.

Let us briefly recall some relevant properties of the Beauville-Bogomolov form on $\mathrm{NS}(M)$ for a smooth projective irreducible symplectic manifold $M$. It is a bilinear form $\operatorname{NS}(M) \times$ $\mathrm{NS}(M) \rightarrow \mathbb{R}$. If $\varrho \in H^{0}\left(M, \Omega_{M}^{2}\right)$ is a global non-degenerate two-form, then there is a constant $c$ such that

$$
\left(D_{1}, D_{2}\right)=c \int_{M} D_{1} D_{2}(\varrho \bar{\varrho})^{\frac{1}{2} \operatorname{dim} M-1} .
$$

Its associated quadratic form is denoted by $q(D)=(D, D)$. It determines the volume of $D$ by $c^{\prime} \int_{M} D^{\operatorname{dim} M}=q(D)^{\frac{1}{2} \operatorname{dim} M}$, for some constant $c^{\prime} \in \mathbb{R}_{>0}$. In particular, a nef divisor $D$ is big if and only if $q(D)>0$ (see also [Huy99, Corollary 3.10]).

As mentioned in the footnote to Definition 4.3, in the case of a K3 surface we will always consider a dual version to the Donaldson morphisms.

Definition 5.4. Let $v \in H_{\mathrm{alg}}^{*}(X, \mathbb{Z})$ be a positive primitive vector and let $\omega \in \operatorname{NS}(X)_{\mathbb{Q}}$ be an ample divisor which is generic with respect to $v$. We define the Mukai homomorphism $\theta_{v}: v^{\perp} \rightarrow \mathrm{NS}\left(M_{\omega}^{\beta}(v)\right)$ by

$$
\theta_{v}(w) \cdot C=\frac{1}{\rho}\left(w, \Phi_{\mathcal{E}}\left(\mathcal{O}_{C}\right)\right)
$$

where $\mathcal{E}$ is a quasi-universal family of similitude $\rho$.

\footnotetext{
${ }^{3}$ We refer to [O'G97] for the notion of a generic polarization; it always exists when $v_{0}$ is positive.
} 
Remark 5.5. With the same arguments as in Proposition 4.4, we can identify the Mukai homomorphism with the Donaldson morphism of Definition 4.3 as follows: given a class $w \in K_{\text {num }}(X)$, we have

$$
\theta_{v}(v(w))=-\lambda_{\mathcal{E}}\left(w^{*}\right) .
$$

The following result is proved in [Yos01b, Sections $7 \&$ 8] (see also [GNY09, Section $1.5])$ :

Theorem 5.6 (Yoshioka). Let $v \in H_{\mathrm{alg}}^{*}(X, \mathbb{Z})$ be a positive primitive vector with $v^{2} \geq 0$. Let $\omega \in \mathrm{NS}(X)_{\mathbb{Q}}$ be an ample divisor which is generic with respect to $v$. Then the Mukai homomorphism induces an isomorphism

$$
\begin{aligned}
& \text { - } \theta_{v}: v^{\perp} \stackrel{\sim}{\rightarrow} \mathrm{NS}\left(M_{\omega}^{\beta}(v)\right) \text {, if } v^{2}>0 ; \\
& \text { - } \theta_{v}: v^{\perp} / v \stackrel{\sim}{\rightarrow} \operatorname{NS}\left(M_{\omega}^{\beta}(v)\right) \text {, if } v^{2}=0 .
\end{aligned}
$$

Under this isomorphism, the quadratic Beauville-Bogomolov form for $\mathrm{NS}\left(M_{\omega}^{\beta}(v)\right)$ coincides with the quadratic form of the Mukai pairing on $X$.

Twisted K3 surfaces. The results in the previous sections can be generalized to twisted $\mathrm{K} 3$ surfaces $^{4}$. Let $(X, \alpha)$ be a twisted K3 surface, with $\alpha \in \operatorname{Br}(X)$.

We denoted by $\operatorname{Coh}(X, \alpha)$ the category of $\alpha$-twisted coherent sheaves on $X$. A twisted Chern character for elements of $\operatorname{Coh}(X, \alpha)$ has been defined ${ }^{5}$ in [HS05]. It takes values in the algebraic part of the twisted Mukai lattice; this will be denoted by $H_{\text {alg }}^{*}(X, \alpha, \mathbb{Z})$.

Given $\omega, \beta \in \mathrm{NS}(X)_{\mathbb{Q}}$ with $\omega$ ample, we can define a notion of stability as in the untwisted case. Moduli of $\beta$-twisted $\omega$-Gieseker semistable $\alpha$-twisted sheaves share the same properties as untwisted sheaves (see [Yos06, Lie07]). In particular, the twisted version of Theorem 5.2 is proved in [Yos06, Theorem 3.16], while Theorem 5.6 (and Remark 5.3,(b)) is proved in [Yos06, Theorem 3.19]. Remark 5.3,(a) can be proved in a similar way.

\section{Review: Stability conditions and moduli spaces for objects on K3 SURFACES}

In this section we give a brief review of Bridgeland's results on stability conditions for K3 surfaces in [Bri08], and of results by Toda, Yoshioka and others related to moduli spaces of Bridgeland-stable objects.

Space of stability conditions for a K3 surface. Let $X$ be a smooth projective K3 surface. Fix $\omega, \beta \in \mathrm{NS}(X)_{\mathbb{Q}}$ with $\omega$ ample.

Let $\mathcal{T}(\omega, \beta) \subset \operatorname{Coh} X$ be the subcategory of sheaves whose HN-filtrations factors (with respect to slope-stability) have $\mu_{\omega, \beta}>0$, and $\mathcal{F}(\omega, \beta)$ the subcategory of sheaves with

\footnotetext{
${ }^{4}$ For the basic theory of twisted K3 surfaces, we refer, for example, to [Căl00]

${ }^{5}$ To be precise, the twisted Chern character and the twisted Mukai lattice depend on the choice of a B-field lift $\beta_{0} \in H^{2}(X, \mathbb{Z})$ of $\alpha$. See also [HMS08, Remark 3.3] for further comments on this.
} 
HN-filtration factors satisfying $\mu_{\omega, \beta} \leq 0$. Next, consider the abelian category

$$
\mathcal{A}(\omega, \beta):=\left\{\begin{array}{ll} 
& \bullet \mathcal{H}^{p}(E)=0 \text { for } p \notin\{-1,0\}, \\
E \in \mathrm{D}^{\mathrm{b}}(X): & \bullet \mathcal{H}^{-1}(E) \in \mathcal{F}(\omega, \beta), \\
& \bullet \mathcal{H}^{0}(E) \in \mathcal{T}(\omega, \beta)
\end{array}\right\}
$$

and the $\mathbb{C}$-linear map

$$
Z_{\omega, \beta}: K_{\text {num }}(X) \rightarrow \mathbb{C}, \quad E \mapsto(\exp (\beta+\sqrt{-1} \omega), v(E))
$$

If $Z_{\omega, \beta}(F) \notin \mathbb{R}_{\leq 0}$ for all spherical sheaves $F \in \operatorname{Coh}(X)$ (e.g., this holds when $\omega^{2}>2$ ), then by [Bri08, Lemma 6.2, Prop. 7.1], the pair $\sigma_{\omega, \beta}=\left(Z_{\omega, \beta}, \mathcal{A}(\omega, \beta)\right)$ defines a stability condition. For objects $E \in \mathcal{A}(\omega, \beta)$, we will denote their phase with respect to $\sigma_{\omega, \beta}$ by $\phi_{\omega, \beta}(E)=\phi(Z(E)) \in(0,1]$. By using the support property, as proved in [Bri08, Proposition 10.3], we can extend the above and define stability conditions $\sigma_{\omega, \beta}$, for $\omega, \beta \in$ $\mathrm{NS}(X)_{\mathbb{R}}$

Denote by $U(X) \subset \operatorname{Stab}(X)$ the open subset consisting of the stability conditions $\sigma_{\omega, \beta}$ just constructed up to the action of $\widetilde{G L}_{2}(\mathbb{R})$. It can also be characterized as the open subset $U(X) \subset \operatorname{Stab}(X)$ consisting of stability conditions for which the skyscraper sheaves $k(x)$ of points are stable of the same phase. Let $\operatorname{Stab}^{\dagger}(X) \subset \operatorname{Stab}(X)$ be the connected component containing $U(X)$. Let $\mathcal{P}(X) \subset H_{\text {alg }}^{*}(X)_{\mathbb{C}}$ be the subset consisting of vectors whose real and imaginary parts span positive definite two-planes in $H_{\text {alg }}^{*}(X)_{\mathbb{R}}$ with respect to the Mukai pairing. It has two connected components, corresponding to the induced orientation of the two-plane. Choose $\mathcal{P}^{+}(X) \subset \mathcal{P}(X)$ as the connected component containing the vector $\left(1, i \omega,-\omega^{2} / 2\right)$, for $\omega \in \mathrm{NS}(X)_{\mathbb{R}}$ the class of an ample divisor. Furthermore, let $\Delta(X):=\left\{s \in H_{\mathrm{alg}}^{*}(X, \mathbb{Z}): s^{2}=-2\right\}$ be the set of spherical classes, and, for $s \in \Delta$,

$$
s_{\mathbb{C}}^{\perp}:=\left\{\Omega \in H_{\text {alg }}^{*}(X)_{\mathbb{C}}:(\Omega, s)=0\right\} .
$$

Finally, set

$$
\mathcal{P}_{0}^{+}(X):=\mathcal{P}^{+}(X) \backslash \bigcup_{s \in \Delta(X)} s_{\mathbb{C}}^{\perp} \subset H_{\text {alg }}^{*}(X)_{\mathbb{C}} .
$$

Since the Mukai pairing (_, $)$ is non-degenerate, we can define $\eta(\sigma) \in H_{\text {alg }}^{*}(X)_{\mathbb{C}}$ for a stability condition $\sigma=(Z, \mathcal{P}) \in \operatorname{Stab}^{\dagger}(X)$ by

$$
\mathcal{Z}(\sigma)\left(\_\right)=\left(\_, \eta(\sigma)\right) \text {. }
$$

Theorem 6.1 (Bridgeland). The map $\eta: \operatorname{Stab}^{\dagger}(X) \rightarrow H_{\mathrm{alg}}^{*}(X)_{\mathbb{C}}$ is a covering map onto its image $\mathcal{P}_{0}^{+}(X)$.

The proof of Theorem 6.1 relies on an explicit description of the boundary $\partial U(X)$ of $U(X)$, see [Bri08, Theorem 12.1]:

Theorem 6.2. Suppose that $\sigma=(Z, \mathcal{P}) \in \partial U(X)$ is a generic point of the boundary of $U(X)$. Then exactly one of the following possibilities holds. 
$\left(A^{+}\right)$There is a rank $r$ spherical vector bundle $A$ such that the only stable factors of the objects $\{k(x): x \in X\}$ in the stability condition $\sigma$ are $A$ and $\mathrm{ST}_{A}(k(x))$. Thus, the Jordan-Hölder filtration of each $k(x)$ is given by

$$
0 \rightarrow A^{\oplus r} \rightarrow k(x) \rightarrow \mathrm{ST}_{A}(k(x)) \rightarrow 0 .
$$

$\left(A^{-}\right)$There is a rank $r$ spherical vector bundle $A$ such that the only stable factors of the objects $\{k(x): x \in X\}$ in the stability condition $\sigma$ are $A[2]$ and $\mathrm{ST}_{A}^{-1}(k(x))$. Thus, the Jordan-Hölder filtration of each $k(x)$ is given by

$$
0 \rightarrow \mathrm{ST}_{A}^{-1}(k(x)) \rightarrow k(x) \rightarrow A^{\oplus r}[2] \rightarrow 0 .
$$

$\left(C_{k}\right)$ There are a nonsingular rational curve $C \subset X$ and an integer $k$ such that $k(x)$ is stable in the stability condition $\sigma$, for $x \notin C$, and such that the Jordan-Hölder filtration of $k(x)$, for $x \in C$, is given by

$$
0 \rightarrow \mathcal{O}_{C}(k+1) \rightarrow k(x) \rightarrow \mathcal{O}_{C}(k)[1] \rightarrow 0 .
$$

A generic point of the boundary is a stability condition which lies on one wall only (in the sense of Proposition 2.3). We also recall that $\mathrm{ST}_{A}$ denotes the spherical twist functor of [ST01] associated to the spherical object $A$.

Remark 6.3. In the boundary of type $\left(C_{k}\right)$, the Mukai vectors of the stable factors of $k(x)$ span a negative semi-definite plane in $H_{\text {alg }}^{*}(X)_{\mathbb{R}}$.

Remark 6.4. As proven in [HMS08, Section 3.1], the results stated in this section extend without any difference to the case of twisted K3 surfaces: Let $(X, \alpha)$ be a twisted K3 surface, $\alpha \in \operatorname{Br}(X)$. Following loc. cit., we define the following objects analogously to the untwisted case: $\operatorname{Stab}^{\dagger}(X, \alpha), U(X, \alpha), \mathcal{P}_{0}^{+}(X, \alpha)$. The statement corresponding to Theorem 6.1 is [HMS08, Proposition 3.10]. Only Theorem 6.2 is not treated explicitly in [HMS08, Section 3.1]. However, the only geometric statement used in the proof in [Bri08, Section 12] is [Muk87, Proposition 2.14], which states that a spherical torsion-free sheaf on a K3 surface is automatically locally free. Mukai's proof carries over without change.

Moduli stacks of semistable objects. Fix $\sigma=(Z, \mathcal{A}) \in \operatorname{Stab}(X), \phi \in \mathbb{R}$, and $v \in$ $H_{\text {alg }}^{*}(X, \mathbb{Z})$. We let $\mathfrak{M}_{\sigma}(v, \phi)$ be the moduli stack of flat families of $\sigma$-semistable objects of class $v$ and phase $\phi$ : its objects are given by complexes $\mathcal{E} \in \mathrm{D}_{S \text {-perf }}(S \times X)$ whose restrictions $\mathcal{E}_{s}$ belong to $\mathcal{P}(\phi)$ and have Mukai vector $v$, for all closed points $s \in S$. We will often omit $\phi$ from the notation; in fact, by acting with an element of $\mathbb{C}$, as in Remark 2.6, and by using Lemma 2.5, we can always assume $\phi=1$ and $\sigma$ algebraic.

Based on results in [Ina02, Lie06] on the stack of objects in $\mathrm{D}^{\mathrm{b}}(X)$, the following theorem is proved in [Tod08, Theorem 1.4 and Section 3]:

Theorem 6.5 (Toda). Let $X$ be a K3 surface and let $\sigma \in \operatorname{Stab}^{\dagger}(X)$. Then $\sigma$-stability is an open property and $\mathfrak{M}_{\sigma}(v, \phi)$ is an Artin stack of finite type over $\mathbb{C}$.

Let $\mathfrak{M}_{\sigma}^{s}(v, \phi) \subseteq \mathfrak{M}_{\sigma}(v, \phi)$ be the open substack parameterizing stable objects. Inaba proved in [Ina02] that $\mathfrak{M}_{\sigma}^{s}(v, \phi)$ is a $\mathbb{G}_{m}$-gerbe over a symplectic algebraic space $M_{\sigma}^{s}(v, \phi)$. Toda's proof is based on the following statement, which will also need directly: 
Lemma 6.6. Fix $\phi \in \mathbb{R}$ and $v \in H_{\mathrm{alg}}^{*}(X, \mathbb{Z})$.

(a) The moduli stack $\mathfrak{M}_{\sigma}(v, \phi)$ satisfies the valuative criterion of universal closedness.

(b) Assume that $\mathfrak{M}_{\sigma}(v, \phi)=\mathfrak{M}_{\sigma}^{s}(v, \phi)$. Then the coarse moduli space $M_{\sigma}(v, \phi)$ is a proper algebraic space.

Proof. As remarked above, we can assume that $\phi=1, Z(v)=-1$, and that $\sigma$ is algebraic. As a consequence, $\mathcal{A}$ is Noetherian. In this case, [AP06, Theorem 4.1.1] implies the lemma.

Moduli spaces of semistable objects. We generalize the results in Section 5 to Bridgeland stability. The key fact is a comparison between Bridgeland and Gieseker stability when the polarization is "large".

Theorem 6.7 ([Bri08, Proposition 14.1] and [Tod08, Section 6]). Let $v \in H_{\mathrm{alg}}^{*}(X, \mathbb{Z})$, and let $\beta \in \mathrm{NS}(X)_{\mathbb{Q}}, H \in \mathrm{NS}(X)$ be classes with $H$ ample and $\mu_{H, \beta}(v)>0$. If we set $\omega=t H$, then $M_{\sigma_{\omega, \beta}}(v)=M_{H}^{\beta}(v)$ for $t \gg 0$.

We will give a precise bound for $t$ in Corollary 9.14. The following generalizes Theorem 5.2 :

Theorem 6.8 (Toda, Yoshioka). Let $v \in H_{\mathrm{alg}}^{*}(X, \mathbb{Z})$. Assume that $v=m v_{0}$, with $m \in$ $\mathbb{Z}_{>0}$ and $v_{0}$ a primitive vector with $v_{0}^{2} \geq-2$. Then $\mathfrak{M}_{\sigma}(v, \phi)(\mathbb{C})$ is non-empty for all $\sigma=(Z, \mathcal{A}) \in \operatorname{Stab}^{\dagger}(X)$ and all $\phi \in \mathbb{R}$ with $Z(v) \in \mathbb{R}_{>0} \cdot e^{i \phi \pi}$.

Proof. Since we are interested in semistable objects, we can assume that $v=v_{0}$ is primitive. Also, since being semistable is a closed condition on $\operatorname{Stab}(X)$, we can assume that $\sigma$ is generic with respect to $v$, so that every $\sigma$-semistable object of class $v$ is stable. Then the Joyce invariant $J(v)$ of [Tod08] is the motivic invariant of the proper coarse moduli space $M_{\sigma}(v)$.

By [Tod08, Theorem 1.4], $J(v)$ does not depend on $\sigma$, and it is invariant under autoequivalences of $\mathrm{D}^{\mathrm{b}}(X)$. Hence, up to acting by the shift functor, tensoring with a line bundle, and the spherical twist $\mathrm{ST}_{\mathcal{O}}$, we can assume that $v$ is positive, and, by using Theorem 6.7, that $J(v)$ is equal to the motivic invariant of the moduli space $M_{H}(v)$ of Gieseker stable sheaves on $X$ with Mukai vector $v$, for a generic polarization $H$. Since $v$ is positive, Theorem 5.2 shows that $M_{H}(v)$ is non-empty. Hence, $J(v)$ is non-trivial, and so $\mathfrak{M}_{\sigma}(w, \phi)(\mathbb{C})$ is non-empty for all $\sigma$.

By Theorem 6.8 and [Ina11], we get the following corollary, which generalizes Remark $5.3,(\mathrm{~b})$.

Corollary 6.9. Let $v \in H_{\mathrm{alg}}^{*}(X, \mathbb{Z})$ be a primitive vector with $v^{2} \geq-2$, and let $\sigma \in$ $\operatorname{Stab}^{\dagger}(X)$ be a generic stability condition with respect to $v$. Then $M_{\sigma}(v)$ is non-empty, consists of stable objects, and it is a smooth proper symplectic algebraic space of dimension $v^{2}+2$.

Finally, we need the following re-writing of Theorem 5.6. The Mukai homomorphism, as defined in Definition 5.4, is well-defined for Bridgeland stability as well, and denoted in the same way, $\theta_{v}: v^{\perp} \rightarrow \mathrm{NS}\left(M_{\sigma}(v)\right)$. 
Theorem 6.10. Let $(X, \alpha)$ be a twisted $K 3$ surface. Let $v \in H_{\mathrm{alg}}^{*}(X, \alpha, \mathbb{Z})$ be a primitive vector with $v^{2} \geq 0$. Let $\sigma \in \operatorname{Stab}^{\dagger}(X, \alpha)$ be a generic stability condition with respect to $v$. Assume that there exist a K3 surface $X^{\prime}$, a Brauer class $\alpha^{\prime} \in \operatorname{Br}\left(X^{\prime}\right)$, a polarization $H^{\prime} \in \mathrm{NS}\left(X^{\prime}\right)$, and a derived equivalence $\Phi: \mathrm{D}^{\mathrm{b}}(X, \alpha) \rightarrow \mathrm{D}^{\mathrm{b}}\left(X^{\prime}, \alpha^{\prime}\right)$ such that

(a) $v^{\prime}=\Phi(v)$ is positive,

(b) $H^{\prime}$ is generic with respect to $v^{\prime}$, and

(c) $M_{\Phi(\sigma)}\left(v^{\prime}\right)$ consists of twisted $H^{\prime}$-Gieseker stable sheaves on $\left(X^{\prime}, \alpha^{\prime}\right)$.

Then $M_{\sigma}(v)$ is an irreducible symplectic projective manifold, and the Mukai homomorphism induces an isomorphism

- $\theta_{v}: v^{\perp} \stackrel{\sim}{\rightarrow} \mathrm{NS}\left(M_{\sigma}(v)\right)$, if $v^{2}>0$;

- $\theta_{v}: v^{\perp} / v \stackrel{\sim}{\rightarrow} \mathrm{NS}\left(M_{\sigma}(v)\right)$, if $v^{2}=0$.

Under this isomorphism, the quadratic Beauville-Bogomolov form for $\mathrm{NS}\left(M_{\sigma}(v)\right)$ coincides with the quadratic form of the Mukai pairing on $X$.

Proof. Since everything is compatible with Fourier-Mukai equivalences, this follows from [Or197, CS07].

\section{K3 Surfaces: Projectivity of MOduli SPACES}

Let $X$ be a smooth projective $\mathrm{K} 3$ surface, and let $v \in H_{\mathrm{alg}}^{*}(X, \mathbb{Z})$.

In the recent preprint [MYY11b], Minamide, Yanagida, and Yoshioka proved the following: if $\operatorname{NS}(X) \cong \mathbb{Z}$ and $\sigma \in \operatorname{Stab}^{\dagger}(X)$ is a generic stability condition with respect to $v$, then there exist another $\mathrm{K} 3$ surface $Y$, a Brauer class $\alpha \in \operatorname{Br}(Y)$, and a derived equivalence $\Phi: \mathrm{D}^{\mathrm{b}}(X) \rightarrow \mathrm{D}^{\mathrm{b}}(Y, \alpha)$ such that the moduli stack $\mathfrak{M}_{\sigma}(v)$ is isomorphic to a moduli stack of (twisted) Gieseker semistable sheaves on $(Y, \alpha)$ via $\Phi$.

In this section, we improve their argument, and we remove the assumption on the rank of the Néron-Severi group. As a consequence, the divisor class $\ell_{\sigma}$ will give an ample divisor on the coarse moduli space.

We write $v=m v_{0} \in H_{\text {alg }}^{*}(X, \mathbb{Z})$, where $m \in \mathbb{Z}_{>0}$, and $v_{0}=(r, c, s)$ is primitive with $v_{0}^{2} \geq-2$. We start by examining the cases in which $v_{0}^{2} \leq 0$.

Lemma 7.1. Assume that $v_{0}^{2}=-2$. Then, for all $\sigma \in \operatorname{Stab}^{\dagger}(X)$ generic with respect to $v$, $\mathfrak{M}_{\sigma}(v)$ admits a coarse moduli space $M_{\sigma}(v)$ consisting of a single point.

Proof. Corollary 6.9 shows that for all generic $\sigma \in \operatorname{Stab}^{\dagger}(X)$, the stack $\mathfrak{M}_{\sigma}\left(v_{0}\right)=$ $\mathfrak{M}_{\sigma}^{s}\left(v_{0}\right) \neq \emptyset$ is a $\mathbb{G}_{m}$-gerbe over a point. The corresponding object $E_{0}$ is spherical, and in particular admits no non-trivial self-extensions. If $m>1$, then $v^{2}<-2$ shows that there cannot exist any stable object with vector $v$. By induction, every semistable object with Mukai vector $v$ must be of the form $E_{0}^{\oplus m}$.

Lemma 7.2. Assume that $v_{0}^{2}=0$. Let $\sigma \in \operatorname{Stab}^{\dagger}(X)$ be a generic stability condition with respect to $v$. 
(a) For $m=1, M_{\sigma}\left(v_{0}\right)$ is a smooth projective $K 3$ surface, and there exist a class $\alpha \in \operatorname{Br}\left(M_{\sigma}\left(v_{0}\right)\right)$ and a derived equivalence

$$
\Phi_{\sigma, v_{0}}: \mathrm{D}^{\mathrm{b}}(X) \stackrel{\sim}{\rightarrow} \mathrm{D}^{\mathrm{b}}\left(M_{\sigma}\left(v_{0}\right), \alpha\right) .
$$

(b) For $m>1$, a coarse moduli space $M_{\sigma}(v)$ exists and

$$
M_{\sigma}(v) \cong \operatorname{Sym}^{m}\left(M_{\sigma}\left(v_{0}\right)\right) .
$$

Proof. Corollary 6.9 shows the non-emptiness. The fact that $M_{\sigma}\left(v_{0}\right)$ is a smooth projective K3 surface and the derived equivalence is a classical result of Mukai and Căldăraru [Muk87, Căl02] for stable sheaves. This can be generalized to stable complexes as follows. Again by Corollary $6.9, M_{\sigma}\left(v_{0}\right)$ is smooth projective symplectic surface. By [Lie06], it also comes equipped with a torsion class in its Brauer group. The equivalence $\Phi_{\sigma, v_{0}}$ follows now from [BO95, Bri99]. This shows (a).

The proof of (b) follows now as in [MYY11b]. Indeed, clearly $\mathfrak{M}_{\sigma}(v) \neq \emptyset$, and the derived equivalence $\Phi$ maps any complex in $\mathfrak{M}_{\sigma}(v)(\mathbb{C})$ in a torsion sheaf on $M_{\sigma}\left(v_{0}\right)$ of dimension 0 and length $m$.

We can now prove Theorem 1.3, based on an idea of Minamide, Yanagida, and Yoshioka. By Lemma 7.1 and Lemma 7.2, we can restrict to the case $v^{2}>0$.

The following result is proved in [MYY11b, Sections 4.1 and 3.4] for abelian surfaces, and for K3 surfaces of Picard rank one. For the convenience of the reader, we give a self-contained proof for arbitrary K3 surfaces:

Lemma 7.3. Let $\sigma=\left(Z_{\sigma}, \mathcal{A}_{\sigma}\right)$ be a generic stability condition with respect to $v$, lying inside a chamber $\mathcal{C}$ with respect to $v$. Then $\mathcal{C}$ contains a dense subset of stability conditions $\tau=\left(Z_{\tau}, \mathcal{A}_{\tau}\right)$ for which there exists a primitive Mukai vector $w$ with $w^{2}=0$ such that:

(a) $Z_{\tau}(w)$ and $Z_{\tau}(v)$ lie on the same ray in the complex plane.

(b) All $\tau$-semistable objects with Mukai vector $w$ are stable, and $M_{\tau}(w)$ is a smooth projective K3 surface.

Proof. Let us consider claim (a). We may assume $Z_{\sigma}(v)=-1$ and restrict our attention to stability conditions $\tau$ with $Z_{\tau}(v)=-1$. Let $Q \subset H_{\text {alg }}^{*}(X)_{\mathbb{R}}$ be the quadric defined by $w^{2}=0$. Due to the signature of the Mukai pairing, there is a real solution $w_{r}$ to the pair of equations $\Im Z_{\sigma}(w)=0$ and $w^{2}=0$. Since $Q$ has a rational point, rational points are dense in $Q$, i.e., there exists $w_{q} \in H_{\mathrm{alg}}^{*}(X)_{\mathbb{Q}}$ arbitrarily close to $w_{r}$ with $w_{q}^{2}=0$. If $w_{q}$ is sufficiently close, and since $w_{q}$ must be linearly independent of $v$, there will be $\tau=\left(Z_{\tau}, \mathcal{A}_{\tau}\right)$ nearby $\sigma$ such that $\Im Z_{\tau}(v)=\Im Z_{\tau}\left(w_{q}\right)=0$ and $\Re Z_{\tau}=\Re Z_{\sigma}$. Replacing $w_{q}$ by the unique primitive integral class $w \in \mathbb{R} \cdot w_{q}$ with $\Re Z_{\tau}(w)<0$ finishes the proof of the first claim.

It remains to show that claim (b) holds, after possibly replacing $w$ and a further deformation of $\tau$. Note that small deformations of $\tau$ in a codimension one submanifold of $\operatorname{Stab}(X)$ will keep property (a) intact. If this contains a stability condition generic with respect to $w$, our claim follows from Lemma 7.2. Otherwise, we can assume that $\tau$ is on a generic point of a wall, and that for $u \in H_{\text {alg }}^{*}(X, \mathbb{Z})$, the complex number $Z(u)$ has the same phase as $Z(v)$ and $Z(w)$ if and only if $u$ is a linear combination of $v$ and $w$. 
Using the Fourier-Mukai transform associated to $M_{\rho}(w)$ for $\rho$ nearby $\tau$ and generic, we can further assume that $w=(0,0,1)$ is the Mukai vector of a point, and that $\rho$ is in a generic boundary point of the geometric chamber $U(X)$ as described in Theorem 6.2. If $M_{\rho}(v)$ is not a fine moduli space, we need to consider $M_{\rho}(v)$ as a twisted K3 surface; see Remark 6.4.

In the case of a wall of type $\left(A^{+}\right)$, let $w^{\prime}$ be the Mukai vector of $\mathrm{ST}_{A}(k(x))$. Since the objects $\mathrm{ST}_{A}(k(x))$ are $\tau$-stable, the stability condition $\tau$ is generic with respect to $w^{\prime}$, we have $w^{\prime 2}=0$, and $Z\left(w^{\prime}\right)$ has the same phase as $Z(v)$. The case $\left(A^{-}\right)$is analogous.

If we are in case $\left(C_{k}\right)$, then as pointed out in Remark 6.3, the Mukai pairing is negative semi-definite on the linear span $\left\langle w, v\left(\mathcal{O}_{C}(k+1)\right)\right\rangle$. However, since $Z\left(\mathcal{O}_{C}(k+1)\right)$ has the same phase as $Z(v)$, this linear span is equal to the linear span $\langle v, w\rangle$ of $v, w$, in contradiction to $v^{2}>0$.

Let $w$ be the Mukai vector from Lemma 7.3. Let $Y:=M_{\tau}(w)$, and let $\alpha \in \operatorname{Br}(Y)$ be a Brauer class so that the choice of a (quasi-)universal family induces a derived equivalence $\Phi: \mathrm{D}^{\mathrm{b}}(X) \stackrel{\sim}{\rightarrow} \mathrm{D}^{\mathrm{b}}(Y, \alpha)$. Consider the stability condition $\tau^{\prime}:=\Phi(\tau) \in \operatorname{Stab}(Y, \alpha)$. By [HS05, Section 5], we can assume that $\tau^{\prime} \in \operatorname{Stab}^{\dagger}(Y, \alpha)$. Then, by construction, for all $F \in \mathfrak{M}_{\tau}(w)(\mathbb{C}), \Phi(F) \cong k(y)$, for some $y \in Y$. Therefore the skyscraper sheaves are all $\tau^{\prime}$-stable with the same phase, namely $\tau^{\prime} \in U(Y, \alpha)$. Up to acting by $\widetilde{\mathrm{GL}}_{2}^{+}(\mathbb{R})$, we can assume that $\tau^{\prime}=\sigma_{\omega^{\prime}, \beta^{\prime}}$, for some $\omega^{\prime}, \beta^{\prime} \in \mathrm{NS}(Y)_{\mathbb{Q}}$, with $\omega^{\prime}$ ample.

Since $Z_{\tau}(v)$ and $Z_{\tau}(w)$ lie on the same ray in the complex plane, we have $Z_{\omega^{\prime}, \beta^{\prime}}(\Phi(v)) \in$ $\mathbb{R}_{<0}$. Note that by the construction of Lemma 7.3 , the stability condition $\tau^{\prime}$ is still generic with respect to $\Phi(v)$. Since $w$ does not lie in a wall for $v, k(y)$ is not a stable factor (with respect to $\left.\tau^{\prime}\right)$ for $\Phi(E)$, for all $E \in \mathfrak{M}_{\tau}(v)(\mathbb{C})$. By definition of the category $\mathcal{A}\left(\omega^{\prime}, \beta^{\prime}\right)$, this shows that $\Phi(E)[-1]$ is a $\alpha$-twisted locally-free sheaf on $Y$, which is $\mu_{\omega^{\prime}, \beta^{\prime} \text {-semistable. }}$

We claim that $\omega^{\prime}$-slope (semi)stability for sheaves of class $-\Phi(v)$ is equivalent to twisted $\omega^{\prime}$-Gieseker (semi)stability: indeed, assume that a sheaf $E^{\prime}$ with $v\left(E^{\prime}\right)=-\Phi(v)$ is slopesemistable. If $F^{\prime} \subset E^{\prime}$ is a saturated subsheaf of the same slope, then $F^{\prime}[1]$ is a subobject of $E^{\prime}[1]$ in $\mathcal{P}_{\omega^{\prime}, \beta^{\prime}}(1)$; since $\tau^{\prime}$ is generic with respect to $\Phi(v)$, this means that $v\left(F^{\prime}\right)$ is proportional to $v\left(E^{\prime}\right)$; hence the twisted Hilbert polynomial of $F^{\prime}$ is proportional to the twisted Hilbert polynomial of $E^{\prime}$, and this will hold independently of the twist $\beta^{\prime}$. In particular, twisted Gieseker stability on $Y$ for $-\Phi(v)$ is equivalent to untwisted Giesekerstability. This shows that $\Phi \circ[-1]$ induces an isomorphism of stacks

$$
\mathfrak{M}_{\tau}(v) \stackrel{\sim}{\rightarrow} \mathfrak{M}_{\omega^{\prime}}(-\Phi(v)),
$$

where, as in Section $5, \mathfrak{M}_{\omega^{\prime}}(-\Phi(v))$ is the moduli stack of Gieseker semistable sheaves on $(Y, \alpha)$. Moreover, the isomorphism preserves $S$-equivalence classes. Hence, a coarse moduli space for $\mathfrak{M}_{\tau}(v)$ exists, since it exists for $\mathfrak{M}_{\omega^{\prime}}(-\Phi(v))$, and it is a normal irreducible projective variety with $\mathbb{Q}$-factorial singularities, by Remark 5.3,(a). This concludes the proof of the first part of Theorem 1.3.

We can now show the second part of Theorem 1.3, namely that $\ell_{\sigma}$ is well-defined on the coarse moduli space $M_{\sigma}(v)$ and it is ample. 
Lemma 7.4. Let $\sigma=(Z, \mathcal{P}) \in \operatorname{Stab}^{\dagger}(X)$ be such that $Z(v)=-1$, and let $w_{\sigma}:=\Im(\eta(\sigma))$. Then $w_{\sigma}^{2}>0$.

Proof. This follows directly from Theorem 6.1 , since $\eta(\sigma) \in \mathcal{P}_{0}^{+}(X)$.

We first deal with the case when $v$ is primitive. Since $\sigma$ generic with respect to $v$, $\mathfrak{M}_{\sigma}(v)=\mathfrak{M}_{\sigma}^{s}(v)$ is a $\mathbb{G}_{m}$-gerbe over $M_{\sigma}(v)$. Moreover, by the first part of Theorem 1.3 and Remark 5.3,(a), $M_{\sigma}(v)$ is a smooth projective irreducible symplectic manifold. Hence, by Remark 4.6, the divisor class $\ell_{\sigma}$ is well-defined on $M_{\sigma}(v)$.

Corollary 7.5. Let $v \in H_{\text {alg }}^{*}(X, \mathbb{Z})$ be a primitive vector with $v^{2} \geq 2$, and $\sigma \in \operatorname{Stab}^{\dagger}(X)$ be generic with respect to $v$. Then the divisor $\ell_{\sigma}$ is ample.

Proof. By Theorem 6.10, $q\left(\ell_{\sigma}\right)=w_{\sigma}^{2}$. By Lemma 7.4, $w_{\sigma}^{2}>0$, and so $\ell_{\sigma}$ is big and has the strong positivity property of Theorem 1.1. As a symplectic projective manifold, $M_{\sigma}(v)$ has trivial canonical bundle; so the Base Point Free Theorem [KM98, Theorem 3.3] implies that (a multiple of) $\ell_{\sigma}$ is globally generated, and hence ample (see also [Huy99, Proposition 6.3]).

The case in which $v$ is not primitive is more delicate, since we do not have a version of Theorem 6.10 available. Instead, we have to use an explicit comparison with determinant line bundles and rely on the GIT construction for dealing with properly semistable objects; we use [HL10, Section 8.1] as a reference for the classical construction.

By the openness and convexity of the ample cone, it is sufficient to prove the ampleness of $\ell_{\sigma}$ for a dense subset of stability conditions in a given chamber. We can therefore assume that $\sigma$ satisfies the properties of the stability condition $\tau$ in Lemma 7.3; let $\Phi$ be the induced derived equivalence $\Phi: \mathrm{D}^{\mathrm{b}}(X) \rightarrow \mathrm{D}^{\mathrm{b}}(Y, \alpha)$.

We will first assume $\alpha=0$. By $(11), \mathfrak{M}_{\Phi(\sigma)}(-\Phi(v))$ consists of $\omega^{\prime}$-Gieseker semistable sheaves on $Y$, where $\omega^{\prime}$ is a generic polarization. By [HL10, Theorem 8.1.5] and [LP05, Théorème $5 \&$ Proposition 6], we know that $\ell_{\Phi(\sigma)}$ defines a divisor class on the coarse moduli space $M_{\Phi(\sigma)}(-\Phi(v))$; this class depends only on $\Phi(\sigma)$, it has the positive property as in Theorem 1.1, and it is compatible with $\ell_{\Phi(\sigma), \mathcal{E}}$ via pull-back. Since, by [Or197, CS07], the equivalence $\Phi$ is of Fourier-Mukai type, and the construction of $\ell_{\sigma}$ is compatible with the convolution of the Fourier-Mukai kernels, $\ell_{\sigma}$ gives a well-defined class on the coarse moduli space $M_{\sigma}(v)$ as well.

We write $-\Phi(v)=(r, c, s)$, Let $\mathcal{L}_{0}, \mathcal{L}_{1}$ be as defined in [HL10, Definition 8.1.9]; after identifying $h$ of [HL10, Section 8.1] with $\omega^{\prime}$, then in our notation we have $\mathcal{L}_{0}=\theta_{-\Phi(v)}((-r, 0, s))$ and $\mathcal{L}_{1}=\theta_{-\Phi(v)}\left(\left(0, r \omega^{\prime}, \omega^{\prime} . c\right)\right)$. It is immediate to check that, up to rescaling and the functor $\Phi \circ[-1], \ell_{\sigma}$ coincides with the class $\mathcal{L}_{1}$. By Theorem 1.1, $\ell_{\sigma}$ is nef. By [HL10, Theorem 8.1.11 \& Remark 8.1.12], the line bundle $\mathcal{L}_{0} \otimes \mathcal{L}_{1}^{\otimes m}$ is ample for $m \gg 0$. Moreover, $\mathcal{L}_{0} \otimes \mathcal{L}_{1}^{\otimes m}$ for $m \gg 0$ are (up to rescaling) induced by a stability conditions arbitrarily close to $\sigma$. Hence we have found a dense subset of stability conditions for which $\ell_{\sigma}$ is ample.

Finally, in case $\alpha \neq 0$, one can use Proposition 2.3.3.6 and Lemma 2.3.2.8 of [Lie07] to reduce to the case $\alpha=0$. This finishes the proof of Theorem 1.3. 


\section{Flops Via WALL-CROSSING}

In this section, we will first discuss the possible phenomena at walls in $\operatorname{Stab}(X)$, and then proceed to prove Theorem 1.4 .

Let $X$ be a smooth projective K3 surface, let $v$ be a primitive Mukai vector with $v^{2} \geq-2$. Consider a wall $W \subset \operatorname{Stab}(X)$ with respect to $v$ in the sense of Proposition 2.3.

Let $\sigma_{0}=\left(Z_{0}, \mathcal{A}_{0}\right) \in W$ be a generic point on the wall. Let $\sigma_{+}=\left(Z_{+}, \mathcal{A}_{+}\right), \sigma_{-}=$ $\left(Z_{-}, \mathcal{A}_{-}\right)$be two algebraic stability conditions in the two adjacent chambers. By the results of the previous section, the two moduli spaces $M_{\sigma_{ \pm}}(v)$ are non-empty, irreducible symplectic projective manifolds. If we choose (quasi-)universal families $\mathcal{E}_{ \pm}$on $M_{\sigma_{ \pm}}(v)$ of $\sigma_{ \pm}$-stable objects, we obtain in particular (quasi-)families of $\sigma_{0}$-semistable objects. Hence, Theorem 4.1 gives us nef divisor classes $\ell_{\sigma_{0}, \mathcal{E}_{ \pm}}$on $M_{\sigma_{ \pm}}(v)$.

There are several possible phenomena at the wall, depending on the codimension of the locus of strictly $\sigma_{0}$-semistable objects, and depending on whether there are curves $C \subset M_{\sigma_{ \pm}}(v)$ of $S$-equivalent objects with respect to $\sigma_{0}$, i.e., curves with $\ell_{\sigma_{0}, \mathcal{E}_{ \pm}} \cdot C=0$. We call the wall $W$

(a) a fake wall there are no curves in $M_{\sigma_{ \pm}}(v)$ of objects that are $S$-equivalent to each other with respect to $\sigma_{0}$,

(b) a totally semistable wall, if $M_{\sigma_{0}}^{s}(v)=\emptyset$,

(c) a flopping wall, if $W$ is not a fake wall and $M_{\sigma_{0}}^{s}(v) \subset M_{\sigma_{ \pm}}(v)$ has complement of codimension at least two,

(d) a bouncing wall, if there is an isomorphism $M_{\sigma_{+}}(v) \cong M_{\sigma_{-}}(v)$ that maps $\ell_{\sigma_{0}, \mathcal{E}_{+}}$to $\ell_{\sigma_{0}, \mathcal{E}_{-}}$, and there are divisors $D_{ \pm} \subset M_{\sigma_{ \pm}}(v)$ that are covered by curves of objects that are $S$-equivalent to each other with respect to $\sigma_{0}$.

Note that a wall can be both fake and totally semistable. In the case of a fake wall, $W$ does not get mapped to a wall of the nef cone. In the case of a bouncing wall, the map $l_{+}: \overline{\mathcal{C}}_{+} \rightarrow N^{1}\left(M_{\sigma_{+}}(v)\right)$ sends $W$ to a boundary of the nef cone of $M_{\sigma_{+}}(v)=M_{\sigma_{-}}(v)$; and so does $l_{-}$. Hence the image of a path crossing the wall $W$ under $l_{ \pm}$will bounce back into the ample cone once it hits the boundary of the nef cone in $N^{1}$. We will see examples of every type of wall in Section 10.

We should point out that the behavior at fake walls and bouncing walls can exhibit different behaviors than the possibilities observed in [CI04] in a different context: in general, the two universal families over $M_{\sigma_{+}}(v), M_{\sigma_{-}}(v)$ do not seem to be related via a derived autoequivalence of the moduli space $M_{\sigma_{+}}(v)=M_{\sigma_{-}}(v)$.

We can assume that $\sigma_{0}$ is algebraic, $Z_{0}(v)=-1$, and $\phi=1$. By Theorem 6.10 and Lemma 7.4, $\ell_{\sigma_{0}, \mathcal{E}_{ \pm}}$has positive self-intersection. Since both $M_{\sigma_{ \pm}}(v)$ have trivial canonical bundles, we can apply the Base Point Free Theorem [KM98, Theorem 3.3], which shows that $\ell_{\sigma_{0}, \mathcal{E}_{ \pm}}$are both semi-ample.

We denote the induced contraction morphism (cf. [Laz04, Theorem 2.1.27]) by

$$
\pi_{\sigma_{ \pm}}: M_{\sigma_{ \pm}}(v) \rightarrow Y_{ \pm}
$$

where $Y_{ \pm}$are normal irreducible projective varieties. We denote the induced ample divisor classes on $Y_{ \pm}$by $\ell_{0, \pm}$. Note that $\pi_{\sigma_{ \pm}}$is an isomorphism if and only if the wall $W$ is a fake 
wall, a divisorial contraction if $W$ is a bouncing wall, and a small contraction if $W$ is a flopping wall.

We would like to say that $Y_{+}=Y_{-}$, and that they are (an irreducible component of) the coarse moduli space of $\sigma_{0}$-semistable objects. The best statement we can prove in general is the following:

Proposition 8.1. The spaces $Y_{ \pm}$have the following universal property: For any proper irreducible scheme $S$ over $\mathbb{C}$, and for any family $\mathcal{E} \in \mathfrak{M}_{\sigma_{0}}(v)(S)$ such that there exists a closed point $s \in S$ for which $\mathcal{E}_{s}=\left.\mathcal{E}\right|_{\{s\} \times X} \in \mathfrak{M}_{\sigma_{ \pm}}(v)(\mathbb{C})$, there exists a finite morphism $q: T \rightarrow S$ and a natural morphism $f_{q^{*} \mathcal{E}}: T \rightarrow Y_{ \pm}$.

Proof. We prove the statement only for $Y_{+}$; the proof for $Y_{-}$is analogous. Let $S$ be a proper scheme, and let $\mathcal{E}$ be a family as above. We can assume $S$ is normal. By Toda's result, Theorem 6.5, there exists an open subset $S^{\prime} \subseteq S$ such that $\mathcal{E}_{s}$ is $\sigma_{+}$-stable, for all $s \in S^{\prime}$. By the universal property for $M_{\sigma_{+}}(v)$, there exists a natural morphism $f_{\mathcal{E}}^{\prime}: S^{\prime} \rightarrow M_{\sigma_{+}}(v)$. This induces a rational morphism $f_{\mathcal{E}}: S \rightarrow Y_{+}$.

Consider a resolution of singularities for $f_{\mathcal{E}}$,

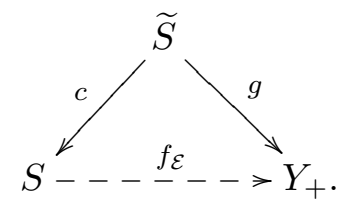

Then, the family $\widetilde{\mathcal{E}}:=(c \times \text { id })^{*} \mathcal{E}$ on $\widetilde{S}$ gives rise to a divisor class $\ell_{\sigma_{0}, \widetilde{\mathcal{E}}}$ on $\widetilde{S}$ such that

$$
\ell_{\sigma_{0}, \widetilde{\mathcal{E}}}=g^{*} \ell_{0,+} \text {. }
$$

Since $\ell_{0,+}$ is ample, $\ell_{\sigma_{0}, \widetilde{\mathcal{E}}}$ is semi-ample. On the other hand, by Theorem 4.1, a curve $C \subseteq \widetilde{S}$ satisfies $\ell_{\sigma_{0}, \widetilde{\mathcal{E}}} \cdot C=0$ if and only if $C$ parameterizes properly $\sigma_{0}$-semistable objects, generically with the same Jordan-Hölder filtration. But every curve in a fiber of $c$ has this property. Hence, up to considering its Stein factorization, the morphism $g$ factorizes through $f_{\mathcal{E}}$, as wanted.

If we can explicitly describe $\sigma_{0}$-semistable objects, Proposition 8.1 shows that $Y_{+}$and $Y_{-}$are actually irreducible components of a coarse moduli space for $\mathfrak{M}_{\sigma_{0}}(v)$. We will see this in some examples in Sections 9 and 10.

Proof. (Theorem 1.4) It remains to prove assertion (b) of Theorem 1.4: in this case, $\mathfrak{M}_{\sigma_{0}}^{s}(v)$ is non-empty, and we can restrict to the case where $\ell_{\sigma_{0}, \mathcal{E}_{ \pm}}$is not ample. By openness of stability, all objects in $\mathfrak{M}_{\sigma_{0}}^{s}(v)(\mathbb{C})$ are stable with respect to $\sigma_{ \pm}$. Write $M_{\sigma_{ \pm}}^{0}(v)$ for the open subsets of $M_{\sigma_{ \pm}}(v)$ consisting of those objects. By assumption, we also have $\operatorname{codim}\left(M_{\sigma_{ \pm}}^{0}(v), M_{\sigma_{ \pm}}(v)\right) \geq 2$. (Note that since $M_{\sigma_{ \pm}}(v)$ are smooth and symplectic, the two conditions $\operatorname{codim}\left(M_{\sigma_{+}}(v) \backslash M_{\sigma_{+}}^{0}(v), M_{\sigma_{+}}(v)\right) \geq 2$ and $\operatorname{codim}\left(M_{\sigma_{-}}(v) \backslash M_{\sigma_{-}}^{0}(v), M_{\sigma_{-}}(v)\right) \geq 2$ are equivalent.)

Consider the birational map

$$
f_{\sigma_{0}}: M_{\sigma_{+}}(v) \rightarrow M_{\sigma-}(v)
$$


induced by the isomorphism $M_{\sigma_{+}}^{0}(v) \stackrel{\sim}{\rightarrow} M_{\sigma_{-}}^{0}(v)$.

Since $\operatorname{codim}\left(M_{\sigma_{ \pm}}(v) \backslash M_{\sigma_{ \pm}}^{0}(v), M_{\sigma_{ \pm}}(v)\right) \geq 2$, and since $M_{\sigma_{ \pm}}(v)$ are projective, numerical divisor classes on $M_{\sigma_{ \pm}}(v)$ are determined by their intersection numbers with curves contained in $M_{\sigma_{ \pm}}^{0}(v)$. Since we can choose (quasi-)universal families $\mathcal{E}_{ \pm}$on $M_{\sigma_{ \pm}}(v)$ that agree on the open subset $M_{\sigma_{ \pm}}^{0}(v)$, this implies that the maps $l_{ \pm}: \overline{\mathcal{C}}^{ \pm} \rightarrow \mathrm{NS}\left(M_{\sigma_{ \pm}}(v)\right)$ are identical, up to analytic continuation and identification of the Néron-Severi groups via $f_{\sigma_{0}}$; more precisely, we have the following equality in $\mathrm{NS}\left(M_{\sigma_{+}}(v)\right)$ :

$$
f_{\sigma_{0}}^{*} \ell_{\sigma_{-}, \mathcal{E}_{-}}=\ell_{Z_{-}, \mathcal{E}_{+}},
$$

where the RHS is given by

$$
\ell_{Z_{-}, \mathcal{E}_{+}}:[C] \mapsto \Im\left(-\frac{Z_{-}\left(\Phi_{\mathcal{E}_{+}}\left(\mathcal{O}_{C}\right)\right)}{Z_{-}(v)}\right)
$$

for all curves $C \subset M_{\sigma_{+}}(v)$. Since $\ell_{\sigma_{0}, \mathcal{E}_{+}}$is not ample, $\ell_{Z_{-}, \mathcal{E}_{+}}$is big and not nef. Hence, the map $f_{\sigma_{0}}$ does not extend to an isomorphism $M_{\sigma_{+}}(v) \stackrel{\sim}{\rightarrow} M_{\sigma_{-}}(v)$. On the other hand, the comparison (12) implies

$$
f_{\sigma_{0}}^{*} \ell_{\sigma_{0}, \mathcal{E}_{-}}=\ell_{\sigma_{0}, \mathcal{E}_{+}} \cdot
$$

As a consequence, we have $Y_{+}=Y_{-}$, and the following diagram commutes:

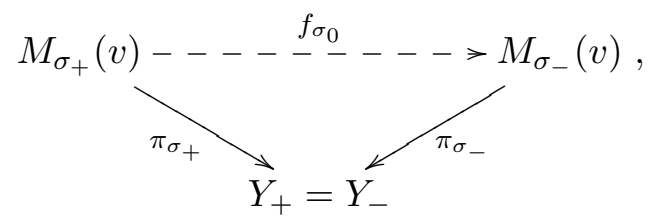

\section{Stable Sheaves ON K3 SURFACES}

In this section we discuss the three main theorems for moduli space of stable sheaves on a K3 surface $X$; for surfaces with Picard group of rank one, some of these examples can also be deduced by [MYY11b, Section 4.3]. We will see that our results, when combined with wellknown methods for explicit wall-crossing computations, already capture a large amount of their geometry. The section is organized as follows: after providing some auxiliary results, we discuss the relation to Lagrangian fibrations; we then study moduli spaces of vector bundles in general, and with complete results for some rank 2 cases; finally, we give a general bound for the ample cone in terms of the Mukai lattice. We start with by recalling the simplest possible case:

Example 9.1. The simplest case is a primitive vector $v$ with $v^{2}=0$. Then Lemma 7.2 and Theorem 6.2 give a complete picture of the possible wall-crossing phenomena. For a generic stability condition, the moduli space is a fixed smooth projective K3 surface $Y$ with a Brauer class $\alpha$, such that $(Y, \alpha)$ is derived equivalent to $X$. The possible walls are derived equivalent to the cases given in Theorem 6.2: In the cases $\left(A^{+}\right)$and $\left(A^{-}\right)$, we have a totally semistable fake wall. In the case $\left(C_{k}\right)$, we get a bouncing wall: the contraction 
induced by the wall is the divisorial contraction of rational (-2)-curves. After we cross the wall, the moduli space is still isomorphic to $Y$, but the universal family gets modified by applying the spherical twist at a line bundle supported on $C$; in $\operatorname{NS}(Y)$, this has the effect of a reflection at $[C]$.

Auxiliary results. We first give an explicit formula for the Mukai vector $w_{\sigma}$ associated to a stability condition.

Lemma 9.2. Let $X$ be a smooth projective $K 3$ surface. Let $v=(r, c, s) \in H_{\mathrm{alg}}^{*}(X, \mathbb{Z})$ be a primitive Mukai vector with $v^{2} \geq-2$, and let $\sigma_{\omega, \beta} \in U(X)$ be a generic stability condition with respect to $v$. Then the divisor class $\ell_{\sigma_{\omega, \beta}} \in N^{1}\left(M_{\sigma_{\omega, \beta}}(v)\right)$ is a positive multiple of $\theta_{v}\left(w_{\sigma_{\omega, \beta}}\right)$, where $w_{\sigma_{\omega, \beta}}=\left(R_{\omega, \beta}, C_{\omega, \beta}, S_{\omega, \beta}\right)$ is given by

$$
\begin{aligned}
& R_{\omega, \beta}=c . \omega-r \beta . \omega \\
& C_{\omega, \beta}=(c . \omega-r \beta . \omega) \beta+\left(s-c . \beta+r \frac{\beta^{2}-\omega^{2}}{2}\right) \omega \\
& S_{\omega, \beta}=c . \omega \frac{\beta^{2}-\omega^{2}}{2}+s \beta . \omega-(c . \beta) \cdot(\beta . \omega) .
\end{aligned}
$$

Proof. Using the Definition of $\ell_{\sigma_{\omega, \beta}}$ in equation (1), and the compatibility of $\theta_{v}$ with the Mukai pairing given in equation (9), we see that the vector is given by

$$
w_{\sigma_{\omega, \beta}}=\Im \frac{e^{i \omega+\beta}}{-\left(e^{i \omega+\beta}, v\right)} \sim_{\mathbb{R}^{+}}-\Im\left(\overline{\left(e^{i \omega+\beta}, v\right)} \cdot e^{i \omega+\beta}\right) .
$$

(Here and in the following $\sim_{\mathbb{R}^{+}}$will mean that the vectors are positive scalar multiples of each other.) Then the claim follows immediately from

$$
e^{i \omega+\beta}=\left(1, \beta, \frac{\beta^{2}-\omega^{2}}{2}\right)+i(0, \omega, \omega \cdot \beta) .
$$

If we write $\omega=t \cdot H$, for an ample divisor $H \in \mathrm{NS}(X)$, we can let $t$ go to zero or $+\infty$. If we take the limit $t \rightarrow 0$ up to rescaling, we obtain a vector $w_{0 \cdot H, \beta}$ with components

$$
\begin{aligned}
& R_{0 \cdot H, \beta}=c . H-r \beta . H \\
& C_{0 \cdot H, \beta}=(c . H-r \beta . H) \beta+\left(s-c . \beta+r \frac{\beta^{2}}{2}\right) H \\
& S_{0 \cdot H, \beta}=c . H \frac{\beta^{2}}{2}+s \beta . H-(c . \beta) \cdot(\beta . H) .
\end{aligned}
$$

If we similarly take the limit $t \rightarrow+\infty$, we obtain a vector $w_{\infty \cdot H, \beta}$ with components

$$
\begin{aligned}
& R_{\infty \cdot H, \beta}=0 \\
& C_{\infty \cdot H, \beta}=-r \frac{H^{2}}{2} H \\
& S_{\infty \cdot H, \beta}=-c . H \frac{H^{2}}{2} .
\end{aligned}
$$


We will also use the following two observations several times; for Lemma 9.3 see, e.g., [BMT11, Section 7.2]; for Lemma 9.4 see [BM11, Lemma 5.9].

Lemma 9.3. Let $\sigma=(Z, \mathcal{A}) \in \operatorname{Stab}(X)$ be a stability condition such that

$$
\gamma:=\inf \{\Im(Z(E))>0: E \in \mathcal{A}\}>0 .
$$

Then an object $E \in \mathcal{A}$ with $\Im(Z(E))=\gamma$ is $\sigma$-stable if and only if $\operatorname{Hom}(\mathcal{P}(1), E)=0$.

The previous lemma applies in particularly when $\Im(Z) \in \mathbb{Z} \cdot \gamma$, for some constant $\gamma>0$. In this case, if an object $E \in \mathcal{A}$ with $\operatorname{Hom}(\mathcal{P}(1), E)=0$ and $\Im(Z(E))=2 \gamma$ is not $\sigma$-stable, then it must be destabilized by a short exact sequence $A \rightarrow E \rightarrow B$ where $A$ and $B$ are $\sigma$-stable with $\Im(Z)=\gamma$.

Lemma 9.4. Let $E \in \mathrm{D}^{\mathrm{b}}(X)$ and $\sigma \in \operatorname{Stab}(X)$ be a stability condition such that $E$ is $\sigma$-semistable. Assume that there is a Jordan-Hölder filtration $M^{\oplus r} \hookrightarrow E \rightarrow N$ of $E$ such that $M, N$ are $\sigma$-stable, $\operatorname{Hom}(E, M)=0$, and $[E]$ and $[M]$ are linearly independent classes in $K_{\text {num }}(X)$. Then $\sigma$ is in the closure of the set of stability conditions where $E$ is stable.

\section{Lagrangian fibrations.}

Example 9.5. In the previous notation, assume $v=(0, c, s)$, for $v$ a primitive vector, $v^{2} \geq 0$, and $c$ a non-zero effective divisor. We assume that $H$ is a generic polarization with respect to $v$. Then, by Theorem 6.7 , for $t$ sufficiently large, $M_{\sigma_{t \cdot H} \beta}(v)=M_{H}^{\beta}(v)$ is a Lagrangian fibration. The semi-ample nef divisor associated to this fibration is given by $w_{\infty \cdot H, \beta} \sim_{\mathbb{R}^{+}}(0,0,-1)$.

The fact that $M_{H}^{\beta}(v)$ is a Lagrangian fibration can be seen by using the divisor $\theta_{v}\left(w_{\infty \cdot H, \beta}\right)$ as follows. By Le Potier's construction, see [LP05, Section 1.3], for all $x \in X$, we can construct a section $s_{x} \in H^{0}\left(M_{H}^{\beta}(v), \theta_{v}\left(w_{\infty \cdot H, \beta}\right)\right)$ via its zero-locus

$$
V\left(s_{x}\right)=\left\{E \in M_{H}^{\beta}(v): \operatorname{Hom}(E, k(x)) \neq 0\right\} .
$$

For any $x$ not contained in the support of $E$, the section $s_{x}$ does not vanish at the point $[E] \in M_{H}^{\beta}(v)$; therefore $\theta_{v}\left(w_{\infty, H, \beta}\right)$ is globally generated by the sections $\left\{s_{x}\right\}_{x \in X}$. The induced morphism contracts the locus of sheaves with fixed support, and thus the image has lower dimension. By Matsushita's Theorem [Mat99, Mat01], the morphism is a Lagrangian fibration.

Remark 9.6. The previous example shows a general phenomenon for nef divisors obtained as an image of a wall in the space of Bridgeland stability conditions. Indeed, by Lemma 7.4 and Theorem 6.10, a divisor $D$ coming from a wall in $\operatorname{Stab}(X)$ must have $q(D)>0$. To obtain a nef divisor $D$ with $q(D)=0$ (which conjecturally corresponds to a Lagrangian fibration), we necessarily have to look at "limit points" in $\operatorname{Stab}(X)$, for example $w_{0 \cdot H, \beta}$, or $w_{\infty \cdot H, \beta}$. We will use these limit points in Examples 10.5 and 10.7. 
Nef cones. In this subsection, we will use wall-crossing for moduli spaces of vector bundles; in particular, in Example 9.8, we will see that for rank two, our main Theorems with an explicit wall-crossing analysis can be sufficient to determine the nef cone of the moduli space.

Example 9.7. Let $X$ be a $\mathrm{K} 3$ surface with $\operatorname{Pic}(X)=\mathbb{Z} \cdot H$, for $H$ an ample line bundle with $H^{2}=2 d, d \geq 1$. Let $v=(r, c H, s)$ be a primitive Mukai vector, with $r, c, s \in \mathbb{Z}, r \geq 0$, $v^{2} \geq-2$. We assume that there exist $A, B \in \mathbb{Z}, A>0$, such that $A c-B r=1$.

Consider the family of stability conditions $\sigma_{t, \frac{B}{A}}:=\sigma_{\omega, \beta}$ on $\mathrm{D}^{\mathrm{b}}(X)$, with $\omega=t \cdot H$ and $\beta:=\frac{B}{A} \cdot H$, for $t>0$. As long as $\sigma_{t, \frac{B}{A}}$ exists, the moduli space $M_{\sigma_{t, \frac{B}{A}}}(v)$ is the moduli space of Gieseker stable sheaves $M_{H}(v)$ : Indeed, we have

$$
\Im\left(Z_{t, \frac{B}{A}}(-)\right) \in \frac{2 t d}{A} \cdot \mathbb{Z}
$$

and $\Im\left(Z_{t, \frac{B}{A}}(v)\right)=\frac{2 t d}{A}$. So Lemma 9.3 shows that Gieseker-stable sheaves are $\sigma_{t, \frac{B}{A}}$-stable.

We distinguish two cases, according to whether $\frac{d B^{2}+1}{A}$ is integral or not. Its relevance is explained by the fact that $w=\left(A, B \cdot H, \frac{d B^{2}+1}{A}\right) \in H_{\mathrm{alg}}^{*}(X)_{\mathbb{Q}}$ is a class with $w^{2}=-2$ and $\Im Z_{t, \frac{B}{A}}(w)=0$; since $A, B$ are coprime, there exists an integral class with these two properties if and only if $\frac{d B^{2}+1}{A}$ is integral.

Case 1: $\frac{d B^{2}+1}{A} \notin \mathbb{Z}$. Then there exists no spherical object with $\Im\left(Z_{t, \frac{B}{A}}(v)\right)=0$. By [Bri08, Proposition 7.1], all values of $t>0$ produce a stability condition. This gives an explicit region of the ample cone of $M_{H}(v)$ :

$$
\left\langle\theta_{v}\left(w_{\sigma_{t, \frac{B}{A}}}\right): t>0\right\rangle \subset \operatorname{Amp}\left(M_{H}(v)\right) .
$$

An explicit computation is in Example 10.5.

Case 2: $\frac{d B^{2}+1}{A} \in \mathbb{Z}$. Then there exists a stable spherical vector bundle $U$ satisfying $\Im\left(Z_{t, \frac{B}{A}}(U)\right)=0$. We let $t_{0}>0$ be such that $\Re\left(Z_{t_{0}}(U)\right)=0$. Then $t>t_{0}$ produces a line segment in the ample cone of $M_{H}(v)$ :

$$
\left\langle\theta_{v}\left(w_{\sigma_{t, \frac{B}{A}}}\right): t>t_{0}\right\rangle \subset \operatorname{Amp}\left(M_{H}(v)\right) .
$$

The question now becomes to understand when $\operatorname{Hom}(U, F) \neq 0$, for $F$ a Gieseker stable sheaf with Mukai vector $v$. An explicit computation is in the following example.

Example 9.8. In the notation of the previous Example 9.7, we take

$$
d=1, \quad v=(2, H, s)(s \leq 0), \quad A=1, \quad B=0 .
$$

Then, the spherical vector bundle $U$ is nothing but $\mathcal{O}_{X}$, and $t_{0}=1$. Up to rescaling, the vector $w_{\sigma_{t, \frac{B}{A}}}$ becomes

$$
w_{\sigma_{t, 0}}=\left(2 t,\left(-2 t^{3}+s t\right) H,-2 t^{3}\right) .
$$


We will see that wall-crossing along this path will naturally lead to contractions of BrillNoether loci, i.e., loci of sheaves $F$ where $h^{0}(F)$ is bigger than expected. These loci and contractions have been studied in [Yos01a]. We distinguish 3 cases.

Case 1: $s=0$. We claim that the nef cone $\operatorname{Nef}\left(M_{H}(v)\right)$ is generated by

$$
\theta_{v}\left(w_{0 \cdot H, 0}\right) \sim_{\mathbb{R}^{+}} \theta_{v}(1,0,0) \text { and } \theta_{v}\left(w_{\infty \cdot H, 0}\right) \sim_{\mathbb{R}^{+}} \theta_{v}(0,-H,-1) .
$$

First of all, observe that any torsion sheaf $T \in \mathfrak{M}_{H}(0, H,-2)(\mathbb{C})$ is a line bundle of degree -1 on a curve of genus 2 ; it follows that there is a short exact sequence

$$
0 \rightarrow \mathcal{O}_{X}^{\oplus 2} \rightarrow \mathrm{ST}_{\mathcal{O}_{X}}^{-1}(T) \rightarrow T \rightarrow 0 .
$$

It easy to see that $F:=\operatorname{ST}_{\mathcal{O}_{X}}^{-1}(T)$ is slope-stable with $v(F)=(2, H, 0)$; hence $\mathrm{ST}_{\mathcal{O}_{X}}^{-1}$ induces an injective morphism $M_{H}(0, H,-2) \rightarrow M_{H}(v)$, which must be an isomorphism (as they have the same dimension). Hence every $F \in \mathfrak{M}_{H}(v)(\mathbb{C})$ is of this form, and $\operatorname{Hom}\left(\mathcal{O}_{X}, F\right)=\mathbb{C}^{2}$, for all $F \in \mathfrak{M}_{H}(v)(\mathbb{C})$.

To compute how the divisor class $\ell_{\sigma_{t, 0}}$ varies when we cross $t=1$, we will use Lemma 9.4. For $0<t<1$, we consider the stability condition $\bar{\sigma}_{t, 0}$ in the boundary of $U(X)$ of type $\left(A^{+}\right)$(see Theorem 6.2). The heart $\mathcal{A}$ for $\bar{\sigma}_{t, 0}$ can be explicitly described (see, e.g., [Yos09, Proposition 2.7] or [BM11, Proposition 5.6]). In particular, $\mathcal{P}(1)$ is generated by $k(x)$ for $x \in X$, by $\mathcal{O}_{X}$, and by all objects of the form $G[1]$, where $G$ is any $\mu$-semistable sheaves of slope 0 satisfying $\operatorname{Hom}\left(\mathcal{O}_{X}, G\right)=0$. Hence, both $\mathcal{O}_{X}$ and, by Lemma 9.3, any $T \in M_{H}(0, H,-2)$ are $\bar{\sigma}_{t, 0}$-stable for all $0<t<1$. Similarly, the short exact sequence

$$
0 \rightarrow T \rightarrow \mathrm{ST}_{\mathcal{O}_{X}}(T) \rightarrow \mathcal{O}_{X}^{\oplus 2} \rightarrow 0
$$

and Lemma 9.3 show that $\mathrm{ST}_{\mathcal{O}_{X}}(T)=\mathrm{ST}_{\mathcal{O}_{X}}^{2}(F)$ is $\bar{\sigma}_{t, 0}$-stable for all $0<t<1$.

In particular, $M_{\bar{\sigma}_{t, 0}}(v)$ for $0<t<1$ is isomorphic to $M_{H}(v)=M_{\sigma_{t, 0}}(v)$ for $1<t$. The universal families are related by an application of $\mathrm{ST}_{\mathcal{O}_{X}}^{2}$; as this acts trivially on the $K$-group, the two families induces the same Mukai homomorphism $v^{\perp} \rightarrow N^{1}\left(M_{H}(v)\right)$.

To understand the wall between the two corresponding chambers, we now consider the path $\sigma_{t,-\epsilon}$, where $\epsilon>0$ is sufficiently small such that $\mathcal{O}_{X}$ and all $T \in \mathfrak{M}_{H}(0, H,-1)(\mathbb{C})$ are both $\sigma_{\frac{1}{2},-\epsilon}$-stable and $\sigma_{2,-\epsilon}$-stable. Note that the subcategory $\mathcal{A}_{t,-\epsilon}$ does not depend on $t$; it is then straightforward to check that $\mathcal{O}_{X}$ and all $T$ are also $\sigma_{t,-\epsilon}$-stable for all $t \in\left[\frac{1}{2}, 2\right]$ : indeed, the imaginary part of $Z_{t,-\epsilon}(w)$ for any Mukai vector $w$ is of the form $t \cdot$ const, and the real part is of the form const + const $\cdot t^{2}$. Then the inequality $\phi_{t,-\epsilon}(w) \leq \phi_{t,-\epsilon}\left(w^{\prime}\right)$ is equivalent to an equation of the form const $\cdot t^{2} \geq$ const.

Let $t_{0} \in\left[\frac{1}{2}, 2\right]$ be such that $\mathcal{O}_{X}$ and $T \in \mathfrak{M}_{H}(0, H,-1)(\mathbb{C})$ have the same phase with respect to $\sigma_{t_{0},-\epsilon}$. Lemma 9.4 shows that $F=\mathrm{ST}_{\mathcal{O}_{X}}^{-1}(T)$ is stable for $t>t_{0}$, and that $\mathrm{ST}_{\mathcal{O}_{X}}^{2}(F)=\mathrm{ST}_{\mathcal{O}_{X}}(T)$ is stable for $t<t_{0}$. This is a totally semistable and fake wall.

For $t \rightarrow 0$, the contraction induced by $w_{0 \cdot H, 0}$ is precisely the Jacobian fibration induced by $\mathrm{ST}_{\mathcal{O}_{X}}$. The wall at $\beta=1 / 2 \cdot H$ corresponds instead to the Uhlenbeck compactification: the corresponding divisorial contraction is induced precisely by $w_{\infty \cdot H, 0}$ (see also [Lo12]).

Case 2: $s=-1$. The nef cone $\operatorname{Nef}\left(M_{H}(v)\right)$ is generated by

$$
\theta_{v}\left(w_{H, 0}\right)=\theta_{v}(2,-3 H,-2) \quad \text { and } \quad \theta\left(w_{\infty \cdot H, 0}\right)=\theta_{v}(0,-H,-1) \text {. }
$$


Similarly to Case 1, the Riemann-Roch Theorem and stability show $\operatorname{Hom}\left(\mathcal{O}_{X}, F\right) \neq 0$, for all $F \in \mathfrak{M}_{H}(v)(\mathbb{C})$. We can use a similar argument as before to find a wall near the singular point $\sigma_{1,0}$ where the Jordan-Hölder filtration of $F$ is given by

$$
H^{0}(F) \otimes \mathcal{O}_{X} \stackrel{\text { ev }}{\longrightarrow} F \rightarrow \text { cone }(\mathrm{ev}) .
$$

There is no stable object with Mukai vector $v$ with respect to $\sigma_{t_{0}, \epsilon}$, hence we are still in the case of a totally semistable wall. Unlike in the previous case, we do have curves of $S$-equivalent objects that get contracted by $w_{H, 0}$ : there is a $\mathbb{P}^{1}$ parameterizing extensions

$$
0 \rightarrow \mathcal{O}_{X} \rightarrow F \rightarrow I_{\Gamma}(H) \rightarrow 0,
$$

for any zero-dimensional subscheme $\Gamma \subset X$ of length 4 contained in a curve $C \in|H|$.

Case 3: $s \leq-2$. The nef cone $\operatorname{Nef}\left(M_{H}(v)\right)$ is generated by

$$
\theta_{v}\left(w_{H, 0}\right)=\theta_{v}(2,(-2+s) H,-2) \quad \text { and } \quad \theta\left(w_{\infty \cdot H, 0}\right)=\theta_{v}(0,-H,-1) .
$$

Indeed, in this case, we will always have both stable objects at $\sigma_{t_{0}, \epsilon}$ (by a dimension count), and strictly semistable ones (corresponding to extensions as in (13), with $\Gamma \subset X$ of length $3-s$ ).

General bound for the ample cone. Finally, we proceed to give an explicit bound for the walls of the "Gieseker chamber" for any Mukai vector $v$, i.e., the chamber for which Bridgeland stability of objects of class $v$ is equivalent to $\beta$-twisted Gieseker stability. In principle, this has been well-known, as all the necessary arguments are already contained in [Bri08, Proposition 14.2]; see also [Tod08, Section 6], [Bay09, Proposition 4.1], [MYY11a, Section 2], [LQ11, Theorem 4.4]; the most explicit results can be found in [Mac12, Sections 2 and 3] (with regards to a slightly different form of the central charge) and [Kaw11]; what follows is essentially a short summary of Kawatani's argument. Corollary 9.14 deduces a general bound for the ample cone from this analysis.

We want to give a bound that is as explicit as possible for the form of the central charge given in (10). Fix a class $\beta \in \mathrm{NS}(X)_{\mathbb{Q}}$, and let $\omega$ vary on a ray in the ample cone. Given a class $v \in H_{\text {alg }}^{*}(X, \mathbb{Z})$ with positive rank and slope, Bridgeland and Toda showed that for $\omega \gg 0$, stable objects of class $v$ are exactly the twisted-Gieseker stable sheaves, see Theorem 6.7. We want to give an explicit bound in terms of $\omega^{2}$ and $\beta, v$ that only depends on the Mukai lattice $H_{\text {alg }}^{*}(X, \mathbb{Z})$.

Definition 9.9. Given divisor classes $\omega, \beta$ with $\omega$ ample, and given a class $v=(r, c, s) \in$ $H_{\text {alg }}^{*}(X, \mathbb{Z})$ with $v^{2} \geq-2$, we write $\left(r, c_{\beta}, s_{\beta}\right)=e^{-\beta}(r, c, s)$ and define its slope $\mu_{\omega, \beta}(v)=$ $\frac{\omega \cdot c_{\beta}}{r}$ as in Section 5, equation (8), and its discrepancy $\delta_{\omega, \beta}(v)$ by

$$
\delta_{\omega, \beta}(v)=-\frac{s_{\beta}}{r}+1+\frac{1}{2} \frac{\mu_{\omega, \beta}(v)^{2}}{\omega^{2}}
$$

Observe that rescaling $\omega$ will rescale $\mu_{\omega, \beta}$ by the same factor, while leaving $\delta_{\omega, \beta}$ invariant. A torsion-free sheaf $F \in \operatorname{Coh} X$ is $\beta$-twisted Gieseker stable if for every subsheaf $G \subset F$ 


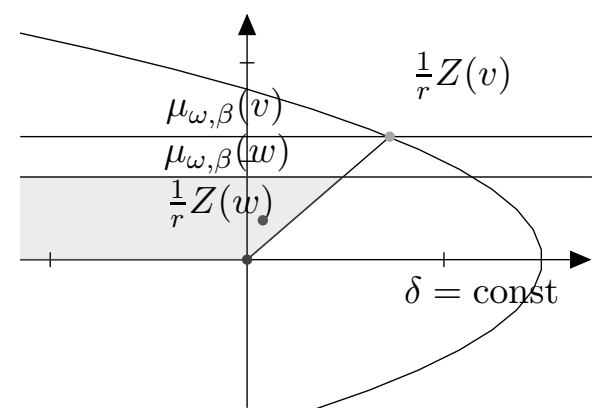

FIgURE 1. Destabilizing subobjects must have smaller $\delta$

we have

$$
\begin{aligned}
& \mu_{\omega, \beta}(G) \leq \mu_{\omega, \beta}(F), \quad \text { and } \\
& \mu_{\omega, \beta}(G)=\mu_{\omega, \beta}(F) \Rightarrow \delta_{\omega, \beta}(G)>\delta_{\omega, \beta}(F) .
\end{aligned}
$$

Combining the Hodge Index theorem with the assumption $v^{2} \geq-2$ shows

$$
\delta_{\omega, \beta}(v) \geq-\frac{s_{\beta}}{r}+1+\frac{c_{\beta}^{2}}{2 r^{2}}=\frac{v^{2}+2}{2 r^{2}}+\left(1-\frac{1}{r^{2}}\right) \geq 0 .
$$

Given a class $v$ with $r>0$, we can write the central charge of equation (10) as

$$
\frac{1}{r} Z_{\omega, \beta}(v)=i \mu_{\omega, \beta}(v)+\frac{\omega^{2}}{2}-\frac{s_{\beta}}{r}=i \mu_{\omega, \beta}(v)+\frac{\omega^{2}}{2}-1-\frac{\mu_{\omega, \beta}(v)^{2}}{2 \omega^{2}}+\delta_{\omega, \beta}(v)
$$

We now fix a class $v \in H_{\text {alg }}^{*}(X, \mathbb{Z})$ with $r(v)>0$ and $\mu_{\omega, \beta}(v)>0$.

Lemma 9.10. Assume $\omega^{2}>2$. Any class $w \in H_{\mathrm{alg}}^{*}(X, \mathbb{Z})$ with $r(w)>0,0<\mu_{\omega, \beta}(w)<$ $\mu_{\omega, \beta}(v)$ such that the phase of $Z_{\omega, \beta}(w)$ is bigger or equal to the phase of $Z_{\omega, \beta}(v)$ satisfies $\delta_{\omega, \beta}(w)<\delta_{\omega, \beta}(v)$.

Proof. By equation (15), it is evident that decreasing $\delta_{\omega, \beta}(v)$ while keeping $\mu_{\omega, \beta}(v)$ fixed will increase the phase of the complex number $Z_{\omega, \beta}(v)$. The same equation also shows that objects with fixed $\delta_{\omega, \beta}$ lie on a parabola, symmetric to the real axis, which intersects the positive real axis; in particular, increasing $\mu_{\omega, \beta}(v)$ while keeping $\delta_{\omega, \beta}(v)$ fixed will also increase the phase of $Z_{\omega, \beta}(v)$; see also fig. 1.

Definition 9.11. Define $D_{v} \subset H_{\text {alg }}^{*}(X, \mathbb{Z})$ as the subset

$$
\left\{w: 0<r(w) \leq r(v), w^{2} \geq-2,0<\mu_{\omega, \beta}(w)<\mu_{\omega, \beta}(v), \delta_{\omega, \beta}(w)<\delta_{\omega, \beta}(v)\right\} .
$$

The set $D_{v}$ is finite: the Hodge Index theorem and $r(w)^{2} \delta_{\omega, \beta}(w)<r(v)^{2} \delta_{\omega, \beta}(v)$ bound the norm of the orthogonal projection of $c_{\beta}(w)$ to $\omega^{\perp} \subset H_{\mathrm{alg}}^{1,1}(X)_{\mathbb{R}}$; the inequality $0<$ $c_{\beta}(w)<r(v) c_{\beta}(v)$ bounds the projection of $c_{\beta}(w)$ to $\mathbb{R} \cdot v$; and, finally, $w^{2} \geq-2$ and $\delta_{\omega, \beta}(w)<\delta_{\omega, \beta}(w)$ give bounds for $s_{\beta}(w)$. We also observe that $D_{v}$ does not change when we rescale $\omega$ in the ray $\mathbb{R}_{>0} \cdot \omega$. 
Definition 9.12. We define $\mu^{\max }(v)$ by

$$
\mu^{\max }(v):=\max \left\{\mu_{\omega, \beta}(w): w \in D_{v}\right\} \cup\left\{\frac{r(v)}{r(v)+1} \cdot \mu_{\omega, \beta}(v)\right\} .
$$

Lemma 9.13. Let $E$ be a $\beta$-twisted Gieseker stable sheaf with $v(E)=v$. If $\omega^{2}>2+$ $\frac{2 \mu^{\max }(v)}{\mu_{\omega, \beta}(v)-\mu^{\max }(v)} \delta_{\omega, \beta}(v)$, then $E$ is $Z_{\omega, \beta}$-stable.

Proof. Consider a destabilizing short exact sequence $A \hookrightarrow E \rightarrow B$ in $\mathcal{A}(\omega, \beta)$ with $\phi_{\omega, \beta}(A) \geq \phi_{\omega, \beta}(E)$. By the long exact cohomology sequence, $A$ is a sheaf. Consider the HN-filtration of $A$ with respect to $\mu_{\omega, \beta}$-slope stability in $\operatorname{Coh} X$, and let $A_{1}, \ldots, A_{n}$ be its HN-filtration factors. Since $A \in \mathcal{A}(\omega, \beta)$ we have $\mu_{\omega, \beta}\left(A_{i}\right)>0$ for all $i$. Since the kernel of $A \rightarrow E$ lies in $\mathcal{F}(\omega, \beta)$, we also have $\mu_{\omega, \beta}\left(A_{i}\right) \leq \mu_{\omega, \beta}\left(A_{1}\right) \leq \mu_{\omega, \beta}(v)$.

By the see-saw property, we can choose an $i$ such that $\phi_{\omega, \beta}\left(A_{i}\right) \geq \phi_{\omega, \beta}(v)$.

First assume $\mu_{\omega, \beta}\left(A_{i}\right)=\mu_{\omega, \beta}(v)$, in which case $i=1$. Consider the composition $g: A_{1} \hookrightarrow$ $A \rightarrow E$ in $\operatorname{Coh} X$. If $g$ is not injective, then $\operatorname{ker} g$ has the same slope $\mu_{\omega, \beta}(\operatorname{ker} g)=\mu_{\omega, \beta}(v)$. Since ker $g \hookrightarrow A$ factors via $\mathcal{H}^{-1}(B) \hookrightarrow A$, this is a contradiction to $\mathcal{H}^{-1}(B) \in \mathcal{F}(\omega, \beta)$. However, if $g$ is injective, $A_{1} \subset E$ is a subsheaf with $\mu_{\omega, \beta}\left(A_{1}\right)=\mu_{\omega, \beta}(E)$ and, by assumption and equation (15), $\delta_{\omega, \beta}\left(A_{1}\right) \leq \delta_{\omega, \beta}(E)$. This contradicts the assumption that $E$ is $\beta$-twisted Gieseker stable.

We have thus proved $\mu_{\omega, \beta}\left(A_{i}\right)<\mu_{\omega, \beta}(v)$. Let $w \in H_{\text {alg }}^{*}(X, \mathbb{Z})$ be the primitive class such that $v\left(A_{i}\right)$ is a positive integer multiple of $w$. We claim that in fact $\mu_{\omega, \beta}(w)=\mu_{\omega, \beta}\left(A_{i}\right) \leq$ $\mu^{\max }(v)$. In case $r(w) \leq r(v)$, this follows from Lemma 9.10 and the definition of the set $D_{v}$. In case $r(w) \geq r(v)+1$, we observe

$$
\omega \cdot c_{\beta}(w) \leq \omega \cdot c_{\beta}\left(A_{i}\right)=\Im Z\left(A_{i}\right) \leq \Im Z(A) \leq \Im Z(E)=\omega \cdot c_{\beta}(v)
$$

to conclude $\mu_{\omega, \beta}(w) \leq \frac{r(v)}{r(v)+1} \cdot \mu_{\omega, \beta}(v)$.

We conclude the proof with a simple geometric argument, see also fig. 2: By equation (15), the phase of $Z(w)$ is less than or equal to the phase $\phi(z)$ of

$$
z:=i \mu^{\max }(v)+\frac{\omega^{2}}{2}-1-\frac{\mu^{\max }(v)^{2}}{2 \omega^{2}} .
$$

We have $\Im \frac{\mu_{\omega, \beta}(v)}{\mu^{\max }(v)} z=\Im \frac{1}{r(v)} Z(v)$ and

$$
\begin{aligned}
\Re \frac{\mu_{\omega, \beta}(v)}{\mu^{\max }(v)} z & =\frac{\omega^{2}}{2}-1+\frac{\mu_{\omega, \beta}(v)-\mu^{\max }(v)}{\mu^{\max }(v)}\left(\frac{\omega^{2}}{2}-1\right)-\frac{\mu_{\omega, \beta}(v) \mu^{\max }(v)}{2 \omega^{2}} \\
& >\frac{\omega^{2}}{2}-1-\frac{\mu_{\omega, \beta}(v)^{2}}{2 \omega^{2}}+\delta_{\omega, \beta}(v)=\Re \frac{1}{r(v)} Z(v) .
\end{aligned}
$$

and thus $\phi(z)<\phi_{\omega, \beta}(v)$. This leads to the contradiction

$$
\phi_{\omega, \beta}(E) \leq \phi_{\omega, \beta}\left(A_{i}\right) \leq \phi(z)<\phi_{\omega, \beta}(E) .
$$




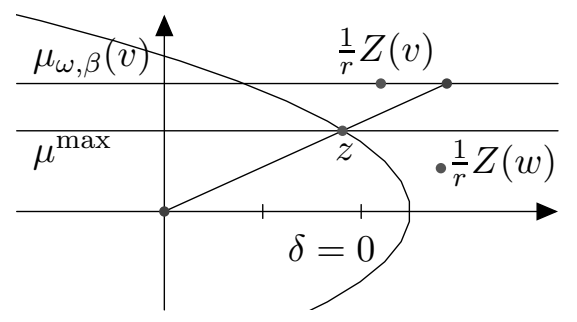

Figure 2. Phases of $Z(w), z$ and $Z(v)$

Corollary 9.14. Let $v \in H_{\mathrm{alg}}^{*}(X, \mathbb{Z})$ be a primitive Mukai vector with $v^{2} \geq 2$. Let $\omega, \beta \in$ $\mathrm{NS}(X)_{\mathbb{Q}}$ be generic with respect to $v$, and such that $r(v)>0$ and $\omega \cdot c_{\beta}(v)>0$. Let $M_{\omega}^{\beta}(v)$ be the moduli space of $\beta$-twisted Gieseker stable sheaves. If $\mu^{\max }(v)$ is as given in Definition 9.12 and $\omega$ satisfies $\omega^{2}>2+\frac{2 \mu^{\max }(v)}{\mu_{\omega, \beta}(v)-\mu^{\max }(v)} \delta_{\omega, \beta}(v)$, then

$$
\theta_{v}\left(w_{\sigma_{\omega, \beta}}\right) \subset \operatorname{Amp}\left(M_{\omega}^{\beta}(v)\right) .
$$

This bound does not depend on the specific K3 surface $X$, other than on its lattice $H_{\text {alg }}^{*}(X, \mathbb{Z})$. We could also make it completely independent of $X$ by considering the full lattice $H^{*}(X, \mathbb{Z})$ instead of $H_{\text {alg }}^{*}(X, \mathbb{Z})$ in Definition 9.11.

\section{Hilbert sCheme of POINTS on K3 SURFACES}

In this section, we study the behavior of our nef divisor at walls for the Hilbert scheme of points on a K3 surface of Picard rank one. Its walls in $\operatorname{Stab}(X)$ have been described partly in [AB13, Section 3], and many of the arguments are identical to the case of $\mathbb{P}^{2}$ treated in [ABCH13].

We will see that our nef divisor can be deformed in one direction to recover the HilbertChow morphism in Example 10.1. In the other direction, we study the first wall in Example 10.2, leading to new results on the Mori cone, see Proposition 10.3 and Remark 10.4. Under more specific numerical constraints, we can continue further to recover a known case and a new case of a well-known conjecture on Lagrangian fibrations, see Theorem 10.6 and Theorem 10.8 .

Let $X$ be a $\mathrm{K} 3$ surface surface with $\operatorname{Pic}(X)=\mathbb{Z} \cdot H$, where $H$ is an ample line bundle. We set $H^{2}=2 d$ for some $d \in \mathbb{Z}, d \geq 1$. As in the previous section, we write $\sigma_{t, b}:=\sigma_{\omega, \beta}$ for $\omega=t H, t>0$ and $\beta=b H$. Let $v=(1,0,1-n)$. Theorem 6.7 implies $M_{\sigma_{t, b}}(v)=\operatorname{Hilb}^{n}(X)$ for $t \gg 0$ and $b<0$. We let $\mathcal{I}$ be the universal family of ideals on $\operatorname{Hilb}^{n}(X)$. We will denote by $\tilde{H} \subset \operatorname{Hilb}^{n}(X)$ the divisor of subschemes intersecting a given curve $C$ in the linear system $|H|$, and by $2 B \subset \operatorname{Hilb}^{n}(X)$ the divisor of non-reduced subschemes; $\tilde{H}$ and $B$ are a basis for $\mathrm{NS}\left(\operatorname{Hilb}^{n}(X)\right)$.

Example 10.1. One wall of the Gieseker chamber is always $b=0$ : In this case, for all $I_{Y} \in \operatorname{Hilb}^{n}(X), I_{Y}[1] \in \mathcal{A}(H, \beta=0), Z\left(I_{Y}[1]\right) \in \mathbb{R}_{<0}$, and the following short exact 
sequence in $\mathcal{P}(1)$ makes $I_{Y}[1]$ strictly semistable:

$$
0 \rightarrow \mathcal{O}_{Y} \rightarrow I_{Y}[1] \rightarrow \mathcal{O}_{X}[1] \rightarrow 0
$$

Further, considering the filtrations of $\mathcal{O}_{Y}, \mathcal{O}_{Y^{\prime}}$ by skyscraper sheaves of points, we see that $I_{Y}$ [1] and $I_{Y^{\prime}}$ [1] are $S$-equivalent if and only if $Y, Y^{\prime}$ define the same point in the Chow variety. It follows that the corresponding nef divisor $\ell_{\sigma_{t, 0}, \mathcal{I}}$ contracts exactly the curves that are contracted by the Hilbert-Chow morphism, and $\ell_{\sigma_{t, 0}, \mathcal{I}} \sim_{\mathbb{R}^{+}} \tilde{H}$.

If $\sigma_{t, \epsilon}$ is a stability condition across the Hilbert-to-Chow wall, $\epsilon>0$, then the moduli space $M_{\sigma_{t, \epsilon}}(v) \cong \operatorname{Hilb}^{n}(X)$ is unchanged, but the universal family is changed: the object $I_{Y}$ is replaced by its derived dual $\mathbf{R} \mathcal{H}$ om $\left(O_{X}, I_{Y}\right)[1]$. This change affects the map $l$ in such a way that the image of a path crossing the Hilbert-to-Chow wall in $\operatorname{Stab}(X)$ will bounce back into the ample cone once it reaches the ray $\mathbb{R}_{>0} \cdot \tilde{H} \subset \mathrm{NS}\left(\operatorname{Hilb}^{n}(X)\right)_{\mathbb{R}}$.

Example 10.2. We consider the path $\sigma_{t, b}$ with $b=-1-\epsilon$ as $t \in(0,+\infty)$ varies; here $\epsilon>0$ is fixed and sufficiently small, and such that there exists no spherical object $U$ with $\Im Z_{t, b}(U)=0$. Let $t_{0}$ be such that $Z_{t_{0}, b}(\mathcal{O}(-H))$, and $Z_{t_{0}, b}(v)$ have the same phase. A direct computation shows $t_{0}=\sqrt{\frac{1}{d}}+O(\epsilon)$, and that for $t>t_{0}$, the phase of $Z_{t, b}(\mathcal{O}(-H))$ is bigger than the phase of $Z_{t, b}(v)$.

We claim that for $t>t_{0}$, any $I_{Y} \in \operatorname{Hilb}^{n}(X)$ is stable. Consider any destabilizing subobject $A \subset I_{Y}$ in $\mathcal{A}_{t, b}=\mathcal{A}(\omega=H, \beta=-H-\epsilon H)$; by the long exact cohomology sequence, $A$ is a torsion-free sheaf. Indeed, if we denote by $B$ the quotient $I_{Y} / A$ in $\mathcal{A}_{t, b}$, we have

$$
0 \rightarrow \mathcal{H}^{-1}(B) \rightarrow \mathcal{H}^{0}(A)=A \rightarrow I_{Y} \rightarrow \mathcal{H}^{0}(B) \rightarrow 0,
$$

and $\mathcal{H}^{-1}(B)$ is torsion-free by definition of the category $\mathcal{A}_{t, b}$.

As in the proof of Lemma 9.13, consider any slope-semistable sheaf $A_{i}$ appearing in the HN-filtration of $A$ with respect to ordinary slope-stability. If we write $v\left(A_{i}\right)=(r, c H, s)$, we have $r>0$ and

$$
\Im Z_{t, b}\left(A_{i}\right)=t \cdot 2 d \cdot(c+r+r \epsilon)
$$

We must have

$$
\Im Z_{t, b}\left(A_{i}\right)<\Im Z_{t, b}\left(I_{Y}\right)=t \cdot 2 d \cdot(1+\epsilon)
$$

and hence $c+r<1$, or $c \leq-r$. This implies

$$
\Im Z_{t, b}\left(A_{i}\right) \leq t \cdot 2 d \cdot r \epsilon \text {. }
$$

Unless $A_{i} \cong O(-H)^{\oplus r}$, the stable factors of $A_{i}$ have rank at least two, and thus $\delta_{t, b}\left(A_{i}\right) \geq \frac{3}{4}$, where $\delta_{t, b}=\delta_{t H, b H}$ is given in Definition 9.9. The same computation that led to equation (15) then shows

$$
\Re Z_{t, b}\left(A_{i}\right) \geq t^{2} d-1+\frac{3}{4}+O(\epsilon) .
$$

This shows that for $t>t_{0}$, the object $A_{i}$ has strictly smaller phase than $I_{Y}$, and so $I_{Y}$ is stable for $t>t_{0}$. 
On the other hand, whenever there is a curve $C \in|H|$ containing $Y$, the short exact sequence

$$
0 \rightarrow \mathcal{O}(-H) \rightarrow I_{Y} \rightarrow \mathcal{O}_{C}(-Y) \rightarrow 0
$$

will make $I_{Y}$ strictly semistable for $t=t_{0}$.

This wall is totally semistable when such a curve exists for any $Y$, that is if and only if $n \leq h^{0}(\mathcal{O}(H))=d+1$. To determine whether this is a fake wall or corresponds to a wall in the nef cone, we would have to determine whether there exists a curve of $S$-equivalent objects. Rather than answering this question in general, we just observe that if there exists a curve $C \in|H|$ for which there exists degree $n$ morphism $g_{n}^{1}: C \rightarrow \mathbb{P}^{1}$, then the pullbacks $\left(g_{n}^{1}\right)^{*}(-x)$ for $x \in \mathbb{P}^{1}$ give a non-trivial family of subschemes $Y \subset C$ for which $\mathcal{O}_{C}(-Y)$ is constant. Thus the corresponding family of ideal sheaves $I_{Y}$, fitting in the exact sequence (16), have a filtration into semistable factors with constant filtration quotients, and hence they are $S$-equivalent.

By [Laz86, Corollary 1.4] and general Brill-Noether theory, a $g_{n}^{1}$ exists for any smooth curve $C \in|H|$ if and only if $n \geq \frac{d+3}{2}$.

Proposition 10.3. Let $X$ be a K3 surface with $\operatorname{Pic} X=\mathbb{Z} \cdot H, H^{2}=2 d$, and $n \geq \frac{d+3}{2}$. The nef cone of $\operatorname{Hilb}^{n}(X)$ is generated by $\tilde{H}$ and $\tilde{H}-\frac{2 d}{d+n} B$.

Proof. Since ideal sheaves are $\sigma_{t, b}$-stable, for $t>t_{0}$, the family $\mathcal{I}$ is a family of $\sigma_{t_{0}, b^{-}}$ semistable objects. Under the assumptions, we have shown above that there exists a curve $R \cong \mathbb{P}^{1} \subset \operatorname{Hilb}^{n}(X)$ parametrizing objects that are $S$-equivalent with respect to $\sigma_{t_{0}, b}$. Theorem 1.1, applied to $\operatorname{Hilb}^{n}(X)$ with $\mathcal{I}$ as a family of $\sigma_{t_{0}, b}$-semistable objects, implies that $\ell_{\sigma_{t_{0}, b}, \mathcal{I}}$ is a nef divisor with $\ell_{\sigma_{t_{0}, b}, \mathcal{I}} \cdot R=0$.

The Hilbert-Chow morphism shows that $\tilde{H}$ is also an extremal ray of the nef cone; it remains to compute the class of $\ell_{\sigma_{t_{0}, b}, \mathcal{I}}$. Since $C$ intersects a general element in $|H|$ in $2 d$ points, and since the linear system given by $R$ will vanish at each point exactly once, we have $R \cdot \tilde{H}=2 d$. On the other hand, $R \cap 2 B$ is the ramification divisor of the map $g_{n}^{1}: C \rightarrow \mathbb{P}^{1}$; the Riemann-Hurwitz formula gives $R .2 B=2 d+2 n$. Since $\ell_{\sigma_{t_{0}, b}, \mathcal{I}} \cdot R=0$, this implies the claim.

For $n$ sufficiently big, the same result could possibly be obtained using the technique of $k$-very ample vector bundles; see [ABCH13, Section 3] and references therein.

The paper [CK12] discusses the existence of Brill-Noether divisors on normalizations of curves with $\delta$ nodes in the linear system $|H|$; the authors ask whether these produce extremal curves of the Mori cone, see [CK12, Question 8.4]. The above proposition answers their question in the case $\delta=0$; it would be interesting to see whether Brill-Noether divisors on nodal curves could also produce curves of $S$-equivalent objects for different walls in $\operatorname{Stab}(X)$ (in the case $n<\frac{d+3}{2}$ ).

Remark 10.4. The Beauville-Bogomolov form, along with the intersection pairing

$$
N_{1}\left(\operatorname{Hilb}^{n}(X)\right) \times N^{1}\left(\operatorname{Hilb}^{n}(X)\right) \rightarrow \mathbb{R},
$$


induces an isomorphism $N_{1} \cong\left(N^{1}\right)^{\vee} \cong N^{1}$. Since $\theta_{v}(0,-H, 0)=\tilde{H}$ and $\theta_{v}(1,0, n-1)=$ $-B$, the Beauville-Bogomolov pairing is determined by

$$
\tilde{H}^{2}=2 d, \quad B^{2}=-2 n+2, \quad \text { and } \quad(H, B)=0 .
$$

(See also [HT10, Section 1].) Thus, the computation in the proof of Proposition 10.3 shows that the isomorphism identifies $R$ with $\tilde{H}+\frac{d+n}{2 n-2} B$. So the self intersection of $R$ (with respect to the Beauville-Bogomolov form) is given by

$$
(R, R)=\tilde{H}^{2}+\left(\frac{d+n}{2 n-2}\right)^{2} B^{2}=2 d-\frac{(d+n)^{2}}{2 n-2}=-\frac{n+3}{2}+\frac{(d+1)(2 n-d-3)}{2 n-2} .
$$

As pointed out to us by Eyal Markman, this does not seem fully consistent with a conjectural description of the Mori cone by Hassett and Tschinkel, see [HT10, Conjecture 1.2]. While $n \geq \frac{d+3}{2}$ implies $(R, R) \geq-\frac{n+3}{2}$ in accordance with their conjecture, in general the Mori cone is smaller than predicted. Let $h, b$ be the primitive curve classes on the rays dual to $\tilde{H}, B$; they are characterized by $h \cdot \tilde{H}=2 d, b \cdot B=1$ and $h \cdot B=b \cdot \tilde{H}=0$. Our extremal curve is give by $R=h+(d+n) b$. If we let $\bar{R}=h+(d+n+1) b$, then

$$
\begin{aligned}
(\bar{R}, \bar{R})=2 d-\frac{(d+n+1)^{2}}{2 n-2} & >-\frac{n+3}{2} \text { for } n \gg 0 \\
\bar{R} \cdot(\tilde{H}-\epsilon B) & >0 .
\end{aligned}
$$

However, since $\bar{R}$. $\left(\tilde{H}-\frac{2 d}{d+n} B\right)<0$, the class $\bar{R}$ cannot be contained in the Mori cone, in contradiction to [HT10, Conjecture 1.2]. The smallest example is the case $d=2$ and $n=5$ and $\bar{R}=h+8 b$, which had been obtained earlier by Markman, [Mar12].

Example 10.5. The previous example considered the case where $n$ is large compared to the genus. Let us now consider a case where the number of points is small compared to the genus: $d=k^{2}(n-1)$ for some integers $k \geq 2$.

With the notation as in the previous examples, we now consider the path of stability conditions $\sigma_{t,-\frac{1}{k}}$ for $t>0$. Then we are in the situation of Example 9.7 with $A=k$ and $B=-1$; more specifically, since $\frac{d+1}{k}$ is not an integer, we are in Case 1 , and the moduli space $M_{\sigma_{t,-\frac{1}{k}}}(v)$ is isomorphic to the Hilbert scheme $\operatorname{Hilb}^{n}(X)$ for all $t>0$. Markushevich and Sawon proved the following result:

Theorem 10.6 ([Mar06, Saw07]). Let $X$ be a K3 surface with $\operatorname{Pic} X=\mathbb{Z} \cdot H, H^{2}=2 d$, and $d=k^{2}(n-1)$ for integers $k \geq 2, n$.

(a) $\operatorname{Nef}\left(\operatorname{Hilb}^{n}(X)\right)$ is generated by

$$
\theta\left(w_{\infty \cdot H,-1 / k}\right)=\theta(0,-H, 0)=\tilde{H} \quad \text { and } \quad \theta\left(w_{0 \cdot H,-1 / k}\right)=\theta(k,-H,(n-1) k)=\tilde{H}-k B .
$$

(b) All nef divisors are semi-ample. The morphism induced by $w_{\infty \cdot H,-1 / k}$ is the Hilbert-to-Chow morphism, while the one induced by $w_{0 \cdot H,-1 / k}$ is a Lagrangian fibration. 
The fact that $w_{\infty \cdot H,-1 / k}$ induces the Hilbert-to-Chow morphism follows in our setup simply from the equality $w_{\infty \cdot H,-1 / k}=w_{\sigma_{t \cdot H, 0}}$ and Example 10.1.

To reprove the existence of the Lagrangian fibration, we can proceed exactly as in [Saw07], except that Bridgeland stability guides and simplifies the arguments: indeed, since $w_{0 \cdot H,-\frac{1}{k}}^{2}=0$, Lemma 7.2 shows that the moduli space $Y:=M_{\sigma_{t,-\frac{1}{k}}}\left(-w_{0 \cdot H,-1 / k}\right)$ is a smooth K3 surface. Let $\Phi$ denote the induced Fourier-Mukai transform $\Phi: \mathrm{D}^{\mathrm{b}}(X) \rightarrow \mathrm{D}^{\mathrm{b}}(Y, \alpha)$; then $\Phi(v)$ has rank 0 . Since $Z_{t,-\frac{1}{k}}\left(-w_{0 \cdot H,-1 / k}\right) \in \mathbb{R}_{<0}$, skyscraper sheaves on $Y$ are $\Phi\left(\sigma_{t,-\frac{1}{k}}\right)$ stable of phase 1 ; hence the stability condition $\Phi\left(\sigma_{t,-\frac{1}{k}}\right)$ is again of the form $\sigma_{\widehat{H}_{t}, \widehat{\beta}_{t}}$ constructed at the beginning of Section 6 , up to the action of $\widetilde{\mathrm{GL}}_{2}^{+}(\mathbb{R})$. Since $\Im Z_{t H,-\frac{1}{k} H}(v)$ is minimal, this has to be true also for $\Im Z_{\widehat{H}_{t}, \widehat{\beta}_{t}}(\Phi(v))$. But then, since the rank of $\Phi(v)$ is zero, this implies that $\widehat{\beta}_{t}=\widehat{\beta}$ is constant in $t$ and $\widehat{H}_{t}=u(t) \widehat{H}$, for some function $u(t)$. We claim that for $t \mapsto 0$, we have $u(t) \mapsto \infty$. Indeed, for $t \mapsto 0$, we must have either $u(t) \mapsto 0$, or $u(t) \mapsto \infty$, and if $u(t) \mapsto 0$, then, by Lemma 9.2, $w_{0 \cdot \widehat{H}, \widehat{\beta}} \neq(0,0,1)$, which is a contradiction.

Hence, for $t \mapsto 0$, via the equivalence $\Phi$, we are in the Gieseker chamber for $Y$. It follows that the moduli space $M_{\sigma_{t, b}}(v) \cong M_{\Phi\left(\sigma_{t, b}\right)}(\Phi(v))$ is isomorphic to a moduli space of twisted Gieseker stable sheaves of rank 0; as is well-known and discussed in Example 9.5, the latter admits a Lagrangian fibration.

Example 10.7. The Hilbert scheme of $n$ points admits a divisor $D$ with $q(D)=0$ if and only if $h^{2} d=k^{2}(n-1)$ for integers $h, k$. The "Tyurin-Bogomolov-Hassett-TschinkelHuybrechts-Sawon Conjecture" would imply that in this case, the Hilbert scheme admits a birational model with a Lagrangian fibration; we refer to [Ver10] for some discussion of the history of the conjecture, and [Bea11] for some context.

We now consider the first unknown case:

Theorem 10.8. Let $X$ be a K3 surface with $\operatorname{Pic} X=\mathbb{Z} \cdot H$ and $H^{2}=2 d$. Assume that there is an odd integer $k$ with $d=\frac{k^{2}}{4}(n-1)$ for some integer $n$. Then:

(a) The movable cone $\operatorname{Mov}\left(\operatorname{Hilb}^{n}(X)\right)$ is generated by

$$
\begin{aligned}
& \theta\left(w_{\infty \cdot H,-\frac{2}{k} \cdot H}\right)=\theta(0,-H, 0)=\tilde{H}, \text { and } \\
& \theta\left(w_{0 \cdot H,-\frac{2}{k} \cdot H}\right)=\theta(k,-2 \cdot H,(n-1) k)=2 \tilde{H}-k B .
\end{aligned}
$$

(b) The morphism induced by $w_{\infty \cdot H,-2 / k}$ is the Hilbert-to-Chow morphism, while the one induced by $w_{0 \cdot H,-2 / k}$ is a Lagrangian fibration on a minimal model for $\operatorname{Hilb}^{n}(X)$.

(c) All minimal models for $\operatorname{Hilb}^{n}(X)$ arise as moduli spaces of stable objects in $\mathrm{D}^{\mathrm{b}}(X)$ and their birational transformations are induced by crossing a wall in $\operatorname{Stab}^{\dagger}(X)$.

Proof. We consider the family of stability conditions $\sigma_{t,-\frac{2}{k}}$ for $t>0$. As in the previous case, the stability condition $\sigma_{t,-\frac{2}{k}}$ exists for all $t>0$, since $\frac{4 d+1}{k} \notin \mathbb{Z}$. We will study the wall-crossing for the moduli spaces $M_{\sigma_{t,-\frac{2}{k}}}(v)$ for $v=(1,0,1-n)$. 
Proceeding as in Example 10.5, we consider the smooth projective K3 surface $Y:=$ $M_{\sigma_{t,-\frac{2}{k}}}\left(-w_{0 \cdot H,-2 / k \cdot H}\right)$ and the induced Fourier-Mukai transform $\Phi: \mathrm{D}^{\mathrm{b}}(X) \rightarrow \mathrm{D}^{\mathrm{b}}(Y, \alpha)$. The assumption $d=\frac{k^{2}}{4}(n-1)$ implies that $\Phi(v)$ has rank 0 , and the same computation as above shows that $t \mapsto 0$ on $X$ corresponds to $t \rightarrow+\infty$ via $\Phi$; in particular, for $t \ll 1$ the stability condition $\sigma_{t,-\frac{2}{k}}$ is in the Gieseker chamber of $Y$ for $\Phi(v)$; thus $M_{0}:=M_{\sigma_{\epsilon,-\frac{2}{k}}}(v)$ admits a Lagrangian fibration, as discussed in Example 9.5.

This also shows that this path meets walls only at finitely many points $t_{1}, \ldots, t_{m} \in \mathbb{R}$. Denote the moduli stacks of stable objects by $\mathfrak{M}_{i}:=\mathfrak{M}_{\sigma t_{i}+\epsilon,-\frac{2}{k}}(v)$, and their coarse moduli spaces by $M_{i}$. By the results in Section 7 , each $M_{i}$ is a smooth irreducible projective variety of dimension $2 n$. We will first prove by descending induction on $i$ that $M_{i}$ is birational to the Hilbert scheme $\operatorname{Hilb}^{n}(X)=M_{m+1}$; this will prove claims (a) and (b).

Let $\left(Z_{i}, \mathcal{A}\right)=\sigma_{i}=\sigma_{t_{i},-\frac{2}{k}}$ be a stability condition on one of the walls. We want to show that the set of $\sigma_{i}$-stable objects of class $v$ is non-empty.

Note that $\Im\left(Z_{t}\right) \in \frac{1}{k} 2 d t \cdot \mathbb{Z}$ and $\Im\left(Z_{t}(v)\right)=\frac{1}{k} 4 d t$ for all $t \in \mathbb{R}$. Thus, if an object $E \in \mathfrak{M}_{i+1}(\mathbb{C})$ is strictly $\sigma_{i}$-semistable, then it fits into a short exact sequence $A \hookrightarrow E \rightarrow B$ in $\mathcal{A}$ where $A, B$ are stable of the same phase, and satisfy

$$
\Im\left(Z_{i}(A)\right)=\Im\left(Z_{i}(B)\right)=\frac{1}{k} 2 d t_{i} .
$$

(In fact we have $Z_{i}(A)=Z_{i}(B)=\frac{1}{2} Z_{i}(v)$, and there are only finitely many classes $v(A), v(B)$ that are possible, see e.g. [Tod08, Lemma 3.15].) Mukai's Lemma, see [Muk87, Corallary 2.8] and [Bri08, Lemma 5.2], shows that

$$
\operatorname{ext}^{1}(E, E) \geq \operatorname{ext}^{1}(A, A)+\operatorname{ext}^{1}(B, B) \text {. }
$$

Since $A \neq B$ are stable and of the same phase, we have $\operatorname{Hom}(A, B)=0=\operatorname{Hom}(B, A)=$ $\operatorname{Ext}^{2}(A, B)$ and thus $-(v(A), v(B))=\chi(A, B)=-\operatorname{ext}^{1}(A, B)$; in particular, $\operatorname{ext}^{1}(A, B)$ is constant as $A, B$ vary in their moduli space of stable objects. Since $A, B, E$ are simple, the bilinearity of $\chi$ then gives

$$
2 \operatorname{ext}^{1}(A, B)=2+\operatorname{ext}^{1}(E, E)-\operatorname{ext}^{1}(A, A)-\operatorname{ext}^{1}(B, B) .
$$

We can distinguish two cases:

(a) $(v(A), v(B))=\operatorname{ext}^{1}(A, B)=1$.

(b) $(v(A), v(B))=\operatorname{ext}^{1}(A, B) \geq 2$.

In case (b), the point on $M_{i+1}$ corresponding to $E$ lies on a rational curve: by Lemma 9.4, each of the extensions parameterized by $\mathbb{P} \operatorname{Ext}^{1}(A, B)$ is $\sigma_{t_{i}+\epsilon,-\frac{2}{k}}$-stable. As $M_{i+1}$ is $K$ trivial, it is not covered by rational curves; this means that a generic object $E \in \mathfrak{M}_{i+1}(\mathbb{C})$ cannot be destabilized by objects of classes $v(A), v(B)$.

We will now proceed to show that case (a) cannot appear. This will imply that there is an open subset $U \subset M_{i+1}$ of objects that are still stable on the wall at $\sigma_{i}$, and thus $U$ gives a common open subset of $M_{i}, M_{i+1}$, inducing a birational map.

So assume we are in case (a). Then $\operatorname{ext}^{1}\left(A^{\prime}, B^{\prime}\right)=\mathbb{C}$ will hold for any pair $A^{\prime} \in$ $\mathfrak{M}_{\sigma_{i}}(v(A))(\mathbb{C}), B^{\prime} \in \mathfrak{M}_{\sigma_{i}}(v(B))(\mathbb{C})$. By Lemma 9.4, the objects $E^{\prime}$ corresponding to the 
unique extension $A^{\prime} \hookrightarrow E^{\prime} \rightarrow B^{\prime}$ will be $\sigma_{t_{i}+\epsilon,-\frac{2}{k}}$-stable; thus the universal family of extensions over $\mathfrak{M}_{\sigma_{i}}(v(A)) \times \mathfrak{M}_{\sigma_{i}}(v(B))$ induces an injective morphism

$$
M_{\sigma_{i}}(v(A)) \times M_{\sigma_{i}}(v(B)) \hookrightarrow M_{i+1}
$$

However, equations (18) and (19) also show that in case (a) we have

$$
\operatorname{ext}^{1}(E, E)=\operatorname{ext}^{1}(A, A)+\operatorname{ext}^{1}(B, B)
$$

in other words the above morphism is an injective morphism between projective varieties of the same dimension. As each $M_{i+1}$ is an irreducible holomorphic symplectic variety, this is a contraction unless one of the moduli spaces on the left is a point, i.e., unless $A$ or $B$ is a spherical object.

Let $\xi$ be the Mukai vector of this spherical object. Then $\xi^{2}=-2$ and the assumption of case (a) implies $(v-\xi, \xi)=1$, and so $(v, \xi)=-1$. If $u=\left(0, \frac{H}{k}, 1-n\right)$, then $\Im Z_{t}\left(\_\right)=$ $t(u, \ldots)$, and so equation (17) implies $(u, \xi)=\frac{1}{2}(u, v)=\frac{1}{2}(n-1)$. By Lemma 10.9, such a class $\xi \in H_{\text {alg }}^{*}(X, \mathbb{Z})$ does not exist. This finishes the proof of claims (a) and (b).

It remains to prove claim (c). By the previous part, wall-crossing induces a chain of birational maps

$$
M_{0} \rightarrow M_{1} \rightarrow \cdots-\rightarrow M_{n}=\operatorname{Hilb}^{n}(X)
$$

As each $M_{i}$ is a smooth $K$-trivial variety, the moduli spaces are isomorphic outside of codimension two, and thus we can canonically identify their Néron-Severi groups:

$$
\mathrm{NS}\left(M_{0}\right)=\mathrm{NS}\left(M_{1}\right)=\cdots=\mathrm{NS}\left(\operatorname{Hilb}^{n}(X)\right) .
$$

Theorem 4.1 produces maps $l_{i}: I_{i} \rightarrow N^{1}\left(\operatorname{Hilb}^{n}(X)\right)$, where $I_{0}=\left(0, t_{0}\right], I_{1}=\left[t_{0}, t_{1}\right]$, etc. By Lemma 10.10 below, the maps $l_{i}, l_{i+1}$ agree on the overlap $t_{i}$; hence they produce a

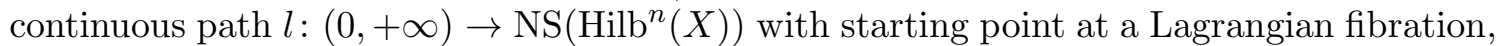
and endpoint at a divisorial contraction. Since $\operatorname{Hilb}^{n}(X)$ has Picard rank two, this path must hit the nef cone of any minimal model of $\operatorname{Hilb}^{n}(X)$.

Lemma 10.9. Let $X$ be K3 surface with assumptions as in Theorem 10.8. Let $u, v \in$ $H_{\mathrm{alg}}^{*}(X, Z)$ be given by $v=(1,0,1-n)$ and $u=\left(0, \frac{H}{k}, 1-n\right)$. Then there exists no class $\xi \in H_{\text {alg }}^{*}(X, Z)$ satisfying the following three equations:

$$
(u, \xi)=\frac{n-1}{2} \quad \text { and } \quad(v, \xi)=-1 \quad \text { and } \quad \xi^{2}=-2 .
$$

Proof. Observe that $v^{2}=2 n-2$, that $(u, v)=n-1$ and $u^{2}=\frac{2 d}{k^{2}}=\frac{1}{2}(n-1)$. Hence the orthogonal complement of $u$ and $v$ is given by $(\mathbb{R} \cdot u+\mathbb{R} \cdot v)^{\perp}=\mathbb{R} \cdot(2 u-v) \subset H_{\text {alg }}^{*}(X)_{\mathbb{R}}$. As $\xi_{0}=\left(\frac{1}{2}, 0, \frac{1}{2}(n+1)\right)$ satisfies the linear equations of the lemma, any solution to the linear equations above is given by $\xi=\xi_{0}+\alpha(2 u-v)$. Since the rank of $\xi$ must be integral, we have $\alpha \in \frac{1}{2}+\mathbb{Z}$.

Using $\xi_{0}^{2}=-\frac{1}{2}(n+1)$ and $\left(\xi_{0}, 2 u-v\right)=n$ as well as $(2 u-v)^{2}=0$, we obtain

$$
\xi^{2}=-2 \Leftrightarrow(4 \alpha-1) \cdot n=-3 .
$$

As $4 \alpha \in \mathbb{Z}$, this is a contradiction to $n \geq 5$. 
Lemma 10.10. Let $X$ be a projective $K 3$ surface, and let $v \in H_{\mathrm{alg}}^{*}(X, \mathbb{Z})$ be a primitive class with $v^{2} \geq-2$. With notation as in Theorem 1.4, assume that there there exists a $\sigma_{0}$-stable objects of class $v$; identify the Néron-Severi groups of $M_{\sigma_{ \pm}}(v)$ by extending the birational morphism $M_{\sigma_{+}}(v) \rightarrow M_{\sigma_{-}}(v)$ induced by the common open subset $M_{\sigma_{0}}^{s}(v)$ to an isomorphism outside of codimension two.

Under this identification, we have an equality $\ell_{\sigma_{0}, \mathcal{E}_{+}}=\ell_{\sigma_{0}, \mathcal{E}_{-}}$in the previous identification $N^{1}\left(M_{\sigma_{+}}(v)\right)=N^{1}\left(M_{\sigma_{-}}(v)\right)$.

The proof is based on the notion of elementary modification in the derived category, introduced by Abramovich and Polishchuk.

Proof. First note that since $M_{\sigma_{ \pm}}(v)$ are $K$-trivial, there exists a common open subset $V \subset M_{\sigma_{ \pm}}(v)$ that contains $M_{\sigma_{0}}^{s}(v)$ and has complement of codimension 2 ; this gives the identification of the Néron-Severi groups.

We can normalize the stability condition $\sigma_{0}=\left(Z_{0}, \mathcal{P}_{0}\right)$ such that $\mathfrak{M}_{\sigma_{ \pm}}(v)$ parameterizes families of $\sigma_{0}$-semistable objects of phase 1 ; we can also assume that $\sigma_{0}$ is algebraic.

Since $M_{\sigma_{ \pm}}(v)$ are projective, $\ell_{\sigma_{0}, \mathcal{E}_{ \pm}}$are determined by their degrees on smooth curves that are contained in $V$ and intersect $M_{\sigma_{0}}^{s}(v)$; let $C \subset V$ be such a curve, and let $U \subset C$ be the open subset $C \cap M_{\sigma_{0}}^{s}(v)$. Since the Brauer group of a curve is trivial, there exists a universal family $\mathcal{E}_{+, C}$ on $C$, corresponding to a lift $C \rightarrow \mathfrak{M}_{\sigma_{+}}(v)$ of the map $C \hookrightarrow V \hookrightarrow$ $M_{\sigma_{+}}(v)$. Similarly, we obtain a family $\mathcal{E}_{-, C}$ via the embedding $C \hookrightarrow V \hookrightarrow M_{\sigma_{-}}(v)$. We can choose $\mathcal{E}_{ \pm, C}$ such that $\left.\left.\mathcal{E}_{+, C}\right|_{U} \cong \mathcal{E}_{-, C}\right|_{U}$. Using further twists by a line bundle, we can assume that this isomorphism extends to a morphism $\varphi: \mathcal{E}_{+, C} \rightarrow \mathcal{E}_{-, C}$ (this follows, e.g., from [Lie06, Proposition 2.2.3]).

We can think of $\mathcal{E}_{ \pm, C}$ as flat families of objects in $\mathcal{P}_{0}(1)$. It is proved in [AP06, Lemma 4.2.3] that the morphism $\varphi$ can be given by a sequence

$$
\mathcal{E}_{+, C}=\mathcal{E}_{0} \rightarrow \mathcal{E}_{1} \rightarrow \cdots \rightarrow \mathcal{E}_{k}=\mathcal{E}_{-, C}
$$

of "elementary modifications" $\mathcal{E}_{i-1} \rightarrow \mathcal{E}_{i}$ : this means that there is a point $c_{i} \in C-U$, an object $Q_{i} \in \mathcal{P}_{0}(1)$ such that $\mathcal{E}_{i-1}$ fits into the short exact sequence

$$
\mathcal{E}_{i-1} \hookrightarrow \mathcal{E}_{i} \rightarrow\left(i_{c_{i}}\right)_{*} Q_{i}
$$

(This is proved in [AP06, Section 4.2] in the case of a smooth affine curve, and $U$ the complement of a point, but the proof extends directly to our case.)

Since $\Im Z_{0}\left(Q_{i}\right)=0$, we obtain

$$
\ell_{\sigma_{0}, \mathcal{E}_{-}} \cdot C=\Im Z_{0}\left(\left(p_{X}\right)_{*} \mathcal{E}_{-, C}\right)=\Im Z_{0}\left(\left(p_{X}\right)_{*} \mathcal{E}_{+, C}\right)+\sum_{i} \Im Z_{0}\left(Q_{i}\right)=\ell_{\sigma_{0}, \mathcal{E}_{+}} \cdot C
$$

as claimed.

Example 10.11. Finally, let us point out that in the case of the Hilbert scheme of $n$ points on an abelian surface $T$ of Picard rank one, Yanagida-Yoshioka and Meachan (in the case of a principally polarized abelian surface) have obtained examples with an infinite series of walls inside the subset $U(T)$ of "geometric" stability conditions; see [Mea12, Section 4] and [YY12, Section 5 and 6]. Their examples have in common that each series of walls 
contains an infinite sequence of bouncing walls, corresponding to divisorial contractions. Between two bouncing walls, there may be finitely many flopping walls; the corresponding line bundle $\ell_{\sigma}$ will traverse finitely many birational models of $\operatorname{Hilb}^{n}(T)$.

\section{REFERENCES}

[AB11] Daniele Arcara and Aaron Bertram. Reider's theorem and Thaddeus pairs revisited. In Grassmannians, moduli spaces and vector bundles, volume 14 of Clay Math. Proc., pages 51-68. Amer. Math. Soc., Providence, RI, 2011. arXiv:0904.3500.

[AB13] Daniele Arcara and Aaron Bertram. Bridgeland-stable moduli spaces for $K$-trivial surfaces. $J$. Eur. Math. Soc. (JEMS), 15(1):1-38, 2013. With an appendix by Max Lieblich. arXiv:0708.2247.

[ABCH13] Daniele Arcara, Aaron Bertram, Izzet Coskun, and Jack Huizenga. The minimal model program for the Hilbert scheme of points on $\mathbb{P}^{2}$ and Bridgeland stability. Adv. Math., 235:580-626, 2013. arXiv:1203.0316.

[AP06] Dan Abramovich and Alexander Polishchuk. Sheaves of $t$-structures and valuative criteria for stable complexes. J. Reine Angew. Math., 590:89-130, 2006. arXiv:math/0309435.

[Bay09] Arend Bayer. Polynomial Bridgeland stability conditions and the large volume limit. Geom. Topol., 13(4):2389-2425, 2009. arXiv:0712.1083.

[Bea11] Arnaud Beauville. Holomorphic symplectic geometry: a problem list. In Complex and differential geometry, volume 8 of Springer Proc. Math., pages 49-63. Springer, Heidelberg, 2011. arXiv:1002.4321.

[BM11] Arend Bayer and Emanuele Macrì. The space of stability conditions on the local projective plane. Duke Math. J., 160(2):263-322, 2011. arXiv:0912.0043.

[BM13] Arend Bayer and Emanuele Macrì. MMP for moduli of sheaves on K3s via wall-crossing: nef and movable cones, Lagrangian fibrations, 2013. arXiv:1301.6968.

[BMT11] Arend Bayer, Emanuele Macrì, and Yukinobu Toda. Bridgeland stability conditions on threefolds I: Bogomolov-Gieseker type inequalities, 2011. To appear in J. Alg. Geom. arXiv:1103.5010.

[BO95] A. Bondal and D. Orlov. Semiorthogonal decomposition for algebraic varieties, 1995. arXiv:alggeom/9506012.

[Bri99] Tom Bridgeland. Equivalences of triangulated categories and Fourier-Mukai transforms. Bull. London Math. Soc., 31(1):25-34, 1999. arXiv:math/9809114.

[Bri02] Tom Bridgeland. Flops and derived categories. Invent. Math., 147(3):613-632, 2002. arXiv:math/0009053.

[Bri07] Tom Bridgeland. Stability conditions on triangulated categories. Ann. of Math. (2), 166(2):317345, 2007. arXiv:math/0212237.

[Bri08] Tom Bridgeland. Stability conditions on K3 surfaces. Duke Math. J., 141(2):241-291, 2008. arXiv:math/0307164.

[Căl00] Andrei Căldăraru. Derived categories of twisted sheaves on Calabi-Yau manifolds. ProQuest LLC, Ann Arbor, MI, 2000. Thesis (Ph.D.)-Cornell University.

[Căl02] Andrei Căldăraru. Nonfine moduli spaces of sheaves on K3 surfaces. Int. Math. Res. Not., 20(20):1027-1056, 2002. arXiv:math/0108180.

[CI04] Alastair Craw and Akira Ishii. Flops of $G$-Hilb and equivalences of derived categories by variation of GIT quotient. Duke Math. J., 124(2):259-307, 2004. arXiv:math/0211360.

[CK12] Ciro Ciliberto and Andreas Leopold Knutsen. On $k$-gonal loci in Severi varieties on general $K 3$ surfaces and rational curves on hyperkähler manifolds, 2012. To appear in J. Math. Pures Appl. arXiv:1204.4838.

[CS07] Alberto Canonaco and Paolo Stellari. Twisted Fourier-Mukai functors. Adv. Math., 212(2):484503, 2007. arXiv:math/0605229. 
[DH98] Igor V. Dolgachev and Yi Hu. Variation of geometric invariant theory quotients. Inst. Hautes Études Sci. Publ. Math., 87(87):5-56, 1998. With an appendix by Nicolas Ressayre. arXiv:alggeom/9402008.

[Don90] S. K. Donaldson. Polynomial invariants for smooth four-manifolds. Topology, 29(3):257-315, 1990.

[EG95] Geir Ellingsrud and Lothar Göttsche. Variation of moduli spaces and Donaldson invariants under change of polarization. J. Reine Angew. Math., 467:1-49, 1995. arXiv:alg-geom/9410005.

[Fal93] Gerd Faltings. Stable G-bundles and projective connections. J. Algebraic Geom., 2(3):507-568, 1993.

[FQ95] Robert Friedman and Zhenbo Qin. Flips of moduli spaces and transition formulas for Donaldson polynomial invariants of rational surfaces. Comm. Anal. Geom., 3(1-2):11-83, 1995. arXiv:alggeom/9410007.

[GHJ03] M. Gross, D. Huybrechts, and D. Joyce. Calabi-Yau manifolds and related geometries. Universitext. Springer-Verlag, Berlin, 2003. Lectures from the Summer School held in Nordfjordeid, June 2001.

[GNY09] Lothar Göttsche, Hiraku Nakajima, and Kōta Yoshioka. K-theoretic Donaldson invariants via instanton counting. Pure Appl. Math. Q., 5(3, Special Issue: In honor of Friedrich Hirzebruch. Part 2):1029-1111, 2009.

[HL10] Daniel Huybrechts and Manfred Lehn. The geometry of moduli spaces of sheaves. Cambridge Mathematical Library. Cambridge University Press, Cambridge, second edition, 2010.

[HMS08] Daniel Huybrechts, Emanuele Macrì, and Paolo Stellari. Stability conditions for generic K3 categories. Compos. Math., 144(1):134-162, 2008. arXiv:math/0608430.

[HS05] Daniel Huybrechts and Paolo Stellari. Equivalences of twisted K3 surfaces. Math. Ann., 332(4):901-936, 2005. arXiv:math/0409030.

[HT01] B. Hassett and Y. Tschinkel. Rational curves on holomorphic symplectic fourfolds. Geom. Funct. Anal., 11(6):1201-1228, 2001. arXiv:math/9910021.

[HT09] Brendan Hassett and Yuri Tschinkel. Moving and ample cones of holomorphic symplectic fourfolds. Geom. Funct. Anal., 19(4):1065-1080, 2009. arXiv:0710.0390.

[HT10] Brendan Hassett and Yuri Tschinkel. Intersection numbers of extremal rays on holomorphic symplectic varieties. Asian J. Math., 14(3):303-322, 2010. arXiv:0909.4745.

[Huy99] Daniel Huybrechts. Compact hyper-Kähler manifolds: basic results. Invent. Math., 135(1):63113, 1999. arXiv:alg-geom/9705025.

[Ina02] Michi-aki Inaba. Toward a definition of moduli of complexes of coherent sheaves on a projective scheme. J. Math. Kyoto Univ., 42(2):317-329, 2002.

[Ina11] Michi-aki Inaba. Smoothness of the moduli space of complexes of coherent sheaves on an abelian or a projective K3 surface. Adv. Math., 227(4):1399-1412, 2011. arXiv:1002.0379.

[Kaw11] Kotaro Kawatani. Stability of Gieseker stable sheaves on $K 3$ surfaces in the sense of Bridgeland and some applications, 2011. arXiv:1103.3921.

[Kin94] A. D. King. Moduli of representations of finite-dimensional algebras. Quart. J. Math. Oxford Ser. (2), 45(180):515-530, 1994.

[KLS06] D. Kaledin, M. Lehn, and Ch. Sorger. Singular symplectic moduli spaces. Invent. Math., 164(3):591-614, 2006. arXiv:math/0504202.

[KM98] János Kollár and Shigefumi Mori. Birational geometry of algebraic varieties, volume 134 of Cambridge Tracts in Mathematics. Cambridge University Press, Cambridge, 1998. With the collaboration of C. H. Clemens and A. Corti, Translated from the 1998 Japanese original.

[KS08] Maxim Kontsevich and Yan Soibelman. Stability structures, motivic Donaldson-Thomas invariants and cluster transformations, 2008. arXiv:0811.2435.

[Kuz06] A. G. Kuznetsov. Hyperplane sections and derived categories. Izv. Ross. Akad. Nauk Ser. Mat., 70(3):23-128, 2006. arXiv:math/0503700. 
[KY11] Masanori Kimura and Kōta Yoshioka. Birational maps of moduli spaces of vector bundles on K3 surfaces. Tokyo J. Math., 34(2):473-491, 2011.

[Laz86] Robert Lazarsfeld. Brill-Noether-Petri without degenerations. J. Differential Geom., 23(3):299307, 1986.

[Laz04] Robert Lazarsfeld. Positivity in algebraic geometry. I, volume 48 of Ergebnisse der Mathematik und ihrer Grenzgebiete. 3. Folge. A Series of Modern Surveys in Mathematics. Springer-Verlag, Berlin, 2004.

[Li93] Jun Li. Algebraic geometric interpretation of Donaldson's polynomial invariants. J. Differential Geom., 37(2):417-466, 1993.

[Li96] Jun Li. Picard groups of the moduli spaces of vector bundles over algebraic surfaces. In Moduli of vector bundles (Sanda, 1994; Kyoto, 1994), volume 179 of Lecture Notes in Pure and Appl. Math., pages 129-146. Dekker, New York, 1996.

[Lie06] Max Lieblich. Moduli of complexes on a proper morphism. J. Algebraic Geom., 15(1):175-206, 2006. arXiv:math/0502198.

[Lie07] Max Lieblich. Moduli of twisted sheaves. Duke Math. J., 138(1):23-118, 2007. arXiv:math/0411337.

[Lo12] Jason Lo. On some moduli of complexes on $K 3$ surfaces, 2012. arXiv:1203.1558.

[LP92] J. Le Potier. Fibré déterminant et courbes de saut sur les surfaces algébriques. In Complex projective geometry (Trieste, 1989/Bergen, 1989), volume 179 of London Math. Soc. Lecture Note Ser., pages 213-240. Cambridge Univ. Press, Cambridge, 1992.

[LP05] Joseph Le Potier. Dualité étrange, sur les surfaces, 2005.

[LQ11] Jason Lo and Zhenbo Qin. Mini-walls for Bridgeland stability conditions on the derived category of sheaves over surfaces, 2011. arXiv:1103.4352.

[Mac12] Antony Maciocia. Computing the walls associated to Bridgeland stability conditions on projective surfaces, 2012. To appear in Asian J. Math. arXiv:1202.4587.

[Mar03] Eyal Markman. On the monodromy of moduli spaces of sheaves on K3 surfaces II, 2003. arXiv:math/0305043.

[Mar06] Dimitri Markushevich. Rational Lagrangian fibrations on punctual Hilbert schemes of $K 3$ surfaces. Manuscripta Math., 120(2):131-150, 2006. arXiv:math/0509346.

[Mar08] Eyal Markman. On the monodromy of moduli spaces of sheaves on K3 surfaces. J. Algebraic Geom., 17(1):29-99, 2008. arXiv:math/0305042.

[Mar11] Eyal Markman. A survey of Torelli and monodromy results for holomorphic-symplectic varieties. In Complex and differential geometry, volume 8 of Springer Proc. Math., pages 257-322. Springer, Heidelberg, 2011. arXiv:1101.4606.

[Mar12] Eyal Markman. Private communication, 2012.

[Mat99] Daisuke Matsushita. On fibre space structures of a projective irreducible symplectic manifold. Topology, 38(1):79-83, 1999. arXiv:alg-geom/9709033.

[Mat01] Daisuke Matsushita. Addendum: "On fibre space structures of a projective irreducible symplectic manifold" [Topology 38 (1999), no. 1, 79-83; MR1644091 (99f:14054)]. Topology, 40(2):431432, 2001. arXiv:math/9903045.

[Mea12] Ciaran Meachan. Moduli of Bridgeland-stable objects, 2012. PhD-Thesis, Unviersity of Edinburgh.

[MM13] Antony Maciocia and Ciaran Meachan. Rank one Bridgeland stable moduli spaces on a principally polarized abelian surface. Int. Math. Res. Not. IMRN, 9:2054-2077, 2013. arXiv:1107.5304.

[MO10] Alina Marian and Dragos Oprea. Generic strange duality for $K 3$ surfaces, 2010. With an appendix by Kōta Yoshioka. arXiv:1005.0102.

[Muk87] S. Mukai. On the moduli space of bundles on $K 3$ surfaces. I. In Vector bundles on algebraic varieties (Bombay, 1984), volume 11 of Tata Inst. Fund. Res. Stud. Math., pages 341-413. Tata Inst. Fund. Res., Bombay, 1987. 
[MW97] Kenji Matsuki and Richard Wentworth. Mumford-Thaddeus principle on the moduli space of vector bundles on an algebraic surface. Internat. J. Math., 8(1):97-148, 1997. arXiv:alggeom/9410016.

[MYY11a] Hiroki Minamide, Shintarou Yanagida, and Kōta Yoshioka. Fourier-mukai transforms and the wall-crossing behavior for Bridgeland's stability conditions, 2011. arXiv:1106.5217.

[MYY11b] Hiroki Minamide, Shintarou Yanagida, and Kōta Yoshioka. Some moduli spaces of Bridgeland's stability conditions, 2011. arXiv:1111.6187.

[O'G97] Kieran G. O'Grady. Moduli of vector-bundles on surfaces. In Algebraic geometry-Santa Cruz 1995, volume 62 of Proc. Sympos. Pure Math., pages 101-126. Amer. Math. Soc., Providence, RI, 1997.

[O'G99] Kieran G. O'Grady. Desingularized moduli spaces of sheaves on a K3. J. Reine Angew. Math., 512:49-117, 1999. arXiv:alg-geom/9708009.

[Or197] D. O. Orlov. Equivalences of derived categories and K3 surfaces. J. Math. Sci. (New York), 84(5):1361-1381, 1997. Algebraic geometry, 7. arXiv:alg-geom/9606006.

[Pol07] A. Polishchuk. Constant families of $t$-structures on derived categories of coherent sheaves. Mosc. Math. J., 7(1):109-134, 167, 2007. arXiv:math/0606013.

[PR11] Arvid Perego and Antonio Rapagnetta. Factoriality properties of moduli spaces of sheaves on abelian and $K 3$ surfaces, 2011. arXiv:1103.3957.

[Saw03] Justin Sawon. Abelian fibred holomorphic symplectic manifolds. Turkish J. Math., 27(1):197230, 2003. arXiv:math/0404362.

[Saw07] Justin Sawon. Lagrangian fibrations on Hilbert schemes of points on K3 surfaces. J. Algebraic Geom., 16(3):477-497, 2007. arXiv:math/0509224.

[ST01] Paul Seidel and Richard Thomas. Braid group actions on derived categories of coherent sheaves. Duke Math. J., 108(1):37-108, 2001. arXiv:math/0001043.

[Tha96] Michael Thaddeus. Geometric invariant theory and flips. J. Amer. Math. Soc., 9(3):691-723, 1996. arXiv:alg-geom/9405004.

[Tod08] Yukinobu Toda. Moduli stacks and invariants of semistable objects on K3 surfaces. Adv. Math., 217(6):2736-2781, 2008. arXiv:math.AG/0703590.

[Tod09] Yukinobu Toda. Limit stable objects on Calabi-Yau 3-folds. Duke Math. J., 149(1):157-208, 2009. arXiv:0803.2356.

[Tod11] Yukinobu Toda. Introduction and open problems of Donaldson-Thomas theory, 2011. To appear in Proceedings of the Conference "Derived Categories", Tokyo 2011.

[Ver10] Misha Verbitsky. HyperKähler SYZ conjecture and semipositive line bundles. Geom. Funct. Anal., 19(5):1481-1493, 2010. arXiv:0811.0639.

[Yos01a] Kōta Yoshioka. Brill-Noether problem for sheaves on K3 surfaces. In Proceedings of the Workshop "Algebraic Geometry and Integrable Systems related to String Theory" (Kyoto, 2000), volume 1232, pages 109-124, 2001.

[Yos01b] Kōta Yoshioka. Moduli spaces of stable sheaves on abelian surfaces. Math. Ann., 321(4):817-884, 2001. arXiv:math/0009001.

[Yos06] Kōta Yoshioka. Moduli spaces of twisted sheaves on a projective variety. In Moduli spaces and arithmetic geometry, volume 45 of Adv. Stud. Pure Math., pages 1-30. Math. Soc. Japan, Tokyo, 2006. arXiv:math/0411538.

[Yos09] Kōta Yoshioka. Stability and the Fourier-Mukai transform. II. Compos. Math., 145(1):112-142, 2009.

[YY12] Shintarou Yanagida and Kōta Yoshioka. Bridgeland's stabilities on abelian surfaces, 2012. arXiv:1203.0884. 
Department of Mathematics, University of Connecticut U-3009, 196 Auditorium Road, STORRs, CT 06269-3009, USA

Current address: School of Mathematics, The University of Edinburgh, James Clerk Maxwell Building, The King's Buildings, Mayfield Road, Edinburgh, Scotland EH9 3JZ, United Kingdom

E-mail address: arend.bayer@ed.ac.uk

URL: http: //www.maths.ed.ac.uk/ abayer/

Department of Mathematics, The Ohio State University, 231 W 18th Avenue, Columbus, OH 43210-1174, USA

E-mail address: macri.6@math.osu.edu

URL: http://www.math.osu.edu/ macri.6/ 